\title{
LATENT NUCLEOPHILIC CARBENES
}

Anatoliy Marchenko, ${ }^{\dagger}$ Georgyi Koidan, ${ }^{\dagger}$ Anastasiya Hurieva, ${ }^{\dagger}$ Kostiantyn Shvydenko, ${ }^{\dagger}$ Alexander

B. Rozhenko, ${ }^{\dagger}{ }^{\text {Eduard B. Rusanov, }}{ }^{\dagger}$ Andrii A. Kyrylchuk, ${ }^{\dagger}$ and Aleksandr Kostyuk $^{\dagger *}$. 


\title{
Supporting information
}

\author{
TABLE OF CONTENTS
}

1. Table S1. Total energy values (E), zero-point energy correction (ZPE) and

Page chemical potential (correction to Gibbs free energy, CP), corrected energy values ( $E+Z P E$ and $E+C P$, a.u.), the lowest vibration frequencies for compounds of 1'a-1'k, 1'a-2'a-TS-1'k-2'k-TS, 2'a-2'k calculated at the RIBP86/TZVP level of approximation.

2. Table S2. Total energy values (E), zero-point energy correction (ZPE) and chemical potential (correction to Gibbs free energy, $C P$ ), corrected energy values ( $E+Z P E$ and $E+C P$, a.u.), the lowest vibration frequencies for singlet and triplet state of carbenes 1'a-1'k, calculated at the RI-BP86/TZVP level of approximation.

3. Table S3. Total energy values (E, RI-CCSD/cc-pVTZ//RI-BP86/TZVP level of approximation), and corrected energy values ( $E+Z P E$ and $E+C P, a . u$ ) for compounds of 1'a-1'k, 1'a-2'a-TS-1'k-2'k-TS, 2'a-2'k using calculated at the RI-BP86/TZVP level of approximation zero-point energy correction (ZPE) and chemical potential (correction to Gibbs free energy, CP).

4. Table S4. Total energy values (E, RI-CCSD/cc-pVTZ//RI-BP86/TZVP level of approximation), and corrected energy values ( $E+Z P E$ and $E+C P, a . u$ ) for singlet and triplet state of carbenes 1'a-1'k using calculated at the RIBP86/TZVP level of approximation zero-point energy correction (ZPE) and chemical potential (correction to Gibbs free energy, CP).

5. Localization and Delocalization Indices for the Compounds 2'a, 2'c.

6. Cartesian coordinates and images for optimized structures $\mathbf{1}^{\prime} \mathbf{a}-\mathbf{1}^{\prime} \mathbf{k}, \mathbf{1}^{\prime} \mathbf{a}-\mathbf{2}^{\mathbf{\prime}} \mathbf{a}$ TS-1'k-2'k-TS, 2'a-2'k (RI-BP86/TZVP).

7. Cartesian coordinates and images for optimized (RI-BP86/TZVP) structures of triplete carbenes $\mathbf{1}^{\prime}$

8. Cartesian coordinates and image for optimized (RI-BP86/TZVP) structure of amidine 2'a-E

9. Thermal ellipsoid plots for crystal structures and the crystal parameters for compounds 7 and 18a. 
Table S1. Total energy values (E), zero-point energy correction (ZPE) and chemical potential (correction to Gibbs free energy, $C P)$, corrected energy values ( $E+Z P E$ and $\mathrm{E}+\mathrm{CP}, \mathrm{a.u}$.), the lowest vibration frequencies for compounds of $1 \mathbf{a}-\mathbf{1} \mathbf{k}$, calculated at the RI-BP86/TZVP level of approximation.

\begin{tabular}{|c|c|c|c|c|c|c|c|c|}
\hline Structure & E, a.u. & ZPE, a.u. & $E+Z P E$, a.u. & $\Delta \mathrm{E}, \mathrm{kcal} \cdot \mathrm{mol}^{-1}$ & $C P$, a.u. & $E+C P$, a.u. & $\Delta \mathrm{G}, \mathrm{kcal} \cdot \mathrm{mol}^{-1}$ & $v, \mathrm{~cm}^{-1}$ \\
\hline 1 'a & -676.746077 & 0.235835 & -676.510242 & 0.00 & 0.190947 & -676.555130 & 0.00 & 34.0 \\
\hline 1'a-2'a-TS & -676.734587 & 0.234732 & -676.499854 & 6.52 & 0.190726 & -676.543861 & 7.07 & -133.4 \\
\hline 2 'a & -676.764281 & 0.236445 & -676.527836 & -11.04 & 0.192839 & -676.571442 & -10.24 & 36.5 \\
\hline 1'b & -907.876144 & 0.314845 & -907.561299 & 0.00 & 0.262422 & -907.613722 & 0.00 & 20.3 \\
\hline $1^{\prime} b-2^{\prime} b-T S$ & -907.864482 & 0.313709 & -907.550773 & 6.61 & 0.261470 & -907.603012 & 6.72 & -114.2 \\
\hline 2'b & -907.894224 & 0.315061 & -907.579163 & -11.21 & 0.261131 & -907.633093 & -12.16 & 4.0 \\
\hline $1 ' c$ & -868.554323 & 0.286491 & -868.267832 & 0.00 & 0.236088 & -868.318234 & 0.00 & 23.9 \\
\hline $1^{\prime} \mathrm{c}-2^{\prime} \mathrm{c}-\mathrm{TS}$ & -868.544412 & 0.285558 & -868.258853 & 5.63 & 0.234230 & -868.310182 & 5.05 & -120.6 \\
\hline 2 'c & -868.579261 & 0.287100 & -868.292161 & -15.27 & 0.237033 & -868.342228 & -15.06 & 27.4 \\
\hline $1 ' d$ & -884.601655 & 0.275302 & -884.326353 & 0.00 & 0.225645 & -884.376010 & 0.00 & 25.5 \\
\hline $1^{\prime} \mathrm{d}-2^{\prime} \mathrm{d}-\mathrm{TS}$ & -884.592797 & 0.274438 & -884.318359 & 5.02 & 0.224647 & -884.368150 & 4.93 & -116.8 \\
\hline 2'd & -884.629434 & 0.275959 & -884.353475 & -17.02 & 0.225610 & -884.403824 & -17.45 & 19.1 \\
\hline 1 'e & -657.335635 & 0.196915 & -657.138720 & 0.00 & 0.154721 & -657.180914 & 0.00 & 43.6 \\
\hline $1^{\prime} \mathrm{e}-2^{\prime} \mathrm{e}-\mathrm{TS}$ & -657.321151 & 0.196329 & -657.124822 & 8.72 & 0.156923 & -657.164228 & 10.47 & -193.8 \\
\hline 2'e & -657.359405 & 0.197508 & -657.161898 & -23.27 & 0.155254 & -657.204151 & -14.58 & 36.8 \\
\hline 1'f & -980.307552 & 0.194336 & -980.113216 & 0.00 & 0.150467 & -980.157086 & 0.00 & 43.0 \\
\hline $\mathbf{1}^{\prime} f-2^{\prime} f-T S$ & -980.296042 & 0.193990 & -980.102052 & 7.01 & 0.151758 & -980.144284 & 8.03 & -142.0 \\
\hline $2^{\prime} f$ & -980.335050 & 0.195647 & -980.139404 & -16.43 & 0.152078 & -980.182973 & -16.24 & 23.4 \\
\hline 1 'g & -582.033891 & 0.190905 & -581.842987 & 0.00 & 0.149842 & -581.884049 & 0.00 & 46.7 \\
\hline 1'g-2'g-TS & -582.025420 & 0.189939 & -581.835481 & 4.71 & 0.148772 & -581.876648 & 4.64 & -162.2 \\
\hline 2'g & -582.072275 & 0.191490 & -581.880785 & -23.72 & 0.149785 & -581.922490 & -24.12 & 28.5 \\
\hline $1 ' h$ & -657.309494 & 0.196781 & -657.112714 & 0.00 & 0.154272 & -657.155223 & 0.00 & 32.9 \\
\hline 1'h-2'h-TS & -657.289609 & 0.195375 & -657.094235 & 11.60 & 0.152264 & -657.137345 & 11.22 & -168.0 \\
\hline 2'h & -657.324683 & 0.196669 & -657.128014 & -9.60 & 0.153955 & -657.170728 & -9.73 & 32.20 \\
\hline $1 ' i$ & -902.588570 & 0.252729 & -902.335841 & 0.00 & 0.201329 & -902.387241 & 0.00 & 42.5 \\
\hline $2 ' i$ & -902.603437 & 0.252623 & -902.350814 & -9.40 & 0.201402 & -902.402035 & -9.28 & 27.7 \\
\hline $1 ' j$ & -878.026304 & 0.288907 & -877.737398 & 0.00 & 0.240434 & -877.785870 & 0.00 & 25.8 \\
\hline $1^{\prime} j-2^{\prime} j-T S$ & -877.995079 & 0.287142 & -877.707936 & 18.49 & 0.238549 & -877.756530 & 18.41 & -241.8 \\
\hline $2 ' j$ & -878.057766 & 0.289230 & -877.768536 & -19.54 & 0.241066 & -877.816699 & -19.35 & 33.4 \\
\hline 1'k & -953.328384 & 0.294386 & -953.033998 & 0.00 & 0.244277 & -953.084106 & 0.00 & 29.8 \\
\hline $1^{\prime} k-2^{\prime} k-T S$ & -953.299728 & 0.293305 & -953.006423 & 17.30 & 0.244174 & -953.055554 & 17.92 & -161.6 \\
\hline 2'k & -953.354297 & 0.294189 & -953.060108 & -16.38 & 0.244871 & -953.109426 & -15.89 & 39.1 \\
\hline
\end{tabular}


Table S2. Total energy values (E), zero-point energy correction (ZPE) and chemical potential (correction to Gibbs free energy, $C P$ ), corrected energy values (E+ZPE and $\mathrm{E}+\mathrm{CP}, \mathrm{a} . \mathrm{u}$.), the lowest vibration frequencies for singlet and triplet state of carbenes $\mathbf{1}^{\prime} \mathbf{a}-\mathbf{1} \mathbf{1} \mathbf{k}$, calculated at the RI-BP86/TZVP level of approximation.

\begin{tabular}{|c|c|c|c|c|c|c|c|c|}
\hline Structure & E, a.u. & ZPE, a.u. & E+ZPE, a.u. & $\Delta \mathrm{E}, \mathrm{kcal} \cdot \mathrm{mol}^{-1}$ & CP, a.u. & $E+C P$, a.u. & $\Delta \mathrm{G}, \mathrm{kcal} \cdot \mathrm{mol}^{-1}$ & $\mathrm{v}, \mathrm{cm}^{-1}$ \\
\hline 1'a & -676.746077 & 0.235835 & -676.510242 & 0.00 & 0.190947 & -676.555130 & 0.00 & 34.0 \\
\hline 1'a-T & -676.706218 & 0.233898 & -676.472320 & 40.13 & 0.188783 & -676.517434 & 40.01 & 28.56 \\
\hline 1'b & -907.876144 & 0.314845 & -907.561299 & 0.00 & 0.262422 & -907.613722 & 0.00 & 20.3 \\
\hline 1'b-T & -907.843725 & 0.312532 & -907.531193 & 40.47 & 0.258793 & -907.584933 & 39.61 & 20.33 \\
\hline 1 'c & -868.554323 & 0.286491 & -868.267832 & 0.00 & 0.236088 & -868.318234 & 0.00 & 23.9 \\
\hline 1'c-T & -868.521950 & 0.285006 & -868.236945 & 40.34 & 0.233773 & -868.288178 & 39.85 & 11.40 \\
\hline 1'd & -884.601655 & 0.275302 & -884.326353 & 0.00 & 0.225645 & -884.376010 & 0.00 & 25.5 \\
\hline $1^{\prime} d-T$ & -884.574431 & 0.273662 & -884.300768 & 38.34 & 0.223135 & -884.351296 & 37.80 & 20.93 \\
\hline 1'e & -657.335635 & 0.196915 & -657.138720 & 0.00 & 0.154721 & -657.180914 & 0.00 & 43.6 \\
\hline 1'e-t & -657.281556 & 0.194634 & -657.086922 & 49.47 & 0.150444 & -657.131112 & 48.23 & 22.06 \\
\hline 1'f & -980.307552 & 0.194336 & -980.113216 & 0.00 & 0.150467 & -980.157086 & 0.00 & 43.0 \\
\hline 1'f-T & -980.274287 & 0.192370 & -980.081916 & 37.25 & 0.146692 & -980.127594 & 36.18 & 17.95 \\
\hline 1'g & -582.033891 & 0.190905 & -581.842987 & 0.00 & 0.149842 & -581.884049 & 0.00 & 46.7 \\
\hline 1'g-T & -582.008683 & 0.189693 & -581.818989 & 28.55 & 0.148368 & -581.860315 & 27.53 & 30.24 \\
\hline 1'h & -657.309494 & 0.196781 & -657.112714 & 0.00 & 0.154272 & -657.155223 & 0.00 & 32.9 \\
\hline 1'h-T & -657.255236 & 0.194850 & -657.060385 & 48.85 & 0.151148 & -657.104087 & 48.10 & 25.70 \\
\hline $1 ' i$ & -902.588570 & 0.252729 & -902.335841 & 0.00 & 0.201329 & -902.387241 & 0.00 & 42.5 \\
\hline $1^{\prime} i-T$ & -902.572895 & 0.251130 & -902.321766 & 42.11 & 0.198240 & -902.374655 & 41.44 & 25.59 \\
\hline 1 'j & -878.026304 & 0.288907 & -877.737398 & 0.00 & 0.240434 & -877.785870 & 0.00 & 25.8 \\
\hline $1^{\prime} \mathrm{j}-\mathrm{T}$ & -877.997768 & 0.286975 & -877.710794 & 40.46 & 0.237307 & -877.760461 & 39.70 & 28.08 \\
\hline 1'k & -953.328384 & 0.294386 & -953.033998 & 0.00 & 0.244277 & -953.084106 & 0.00 & 29.8 \\
\hline 1'k-T & -953.324713 & 0.292717 & -953.031996 & 39.36 & 0.242072 & -953.082641 & 38.96 & 30.60 \\
\hline
\end{tabular}


Table S3. Total energy values (E, RI-CCSD/cc-pVTZ//RI-BP86/TZVP level of approximation), and corrected energy values (E+ZPE and E+CP, a.u) for compounds of 1a$\mathbf{1 k}$ using calculated at the RI-BP86/TZVP level of approximation zero-point energy correction (ZPE) and chemical potential (correction to Gibbs free energy, CP).

\begin{tabular}{|c|c|c|c|c|c|c|c|}
\hline Structure & E, a.u. & ZPE, a.u. & $\mathrm{E}+\mathrm{ZPE}$, a.u. & $\Delta \mathrm{E}, \mathrm{kcal} \cdot \mathrm{mol}^{-1}$ & CP, a.u. & $\mathrm{E}+\mathrm{CP}$, a.u. & $\Delta \mathrm{G}, \mathrm{kcal} \cdot \mathrm{mol}^{-1}$ \\
\hline 1'a & -674.939763 & 0.235835 & -674.703928 & 0.00 & 0.190947 & -674.748816 & 0.00 \\
\hline 1'a-2'a-TS & -674.923512 & 0.234732 & -674.688779 & 9.51 & 0.190726 & -674.732786 & 10.06 \\
\hline 2'a & -674.958211 & 0.236445 & -674.721766 & -11.19 & 0.192839 & -674.765371 & -10.39 \\
\hline 1'b & -905.306880 & 0.314845 & -904.992035 & 0.00 & 0.262422 & -905.044458 & 0.00 \\
\hline $1^{\prime} b-2^{\prime} b-T S$ & -905.289236 & 0.313709 & -904.975527 & 10.36 & 0.261470 & -905.027765 & 10.47 \\
\hline 2'b & -905.322650 & 0.315061 & -905.007589 & -9.76 & 0.261131 & -905.061519 & -10.71 \\
\hline $1 ' c$ & -866.117869 & 0.286491 & -865.831379 & 0.00 & 0.236088 & -865.881781 & 0.00 \\
\hline $1^{\prime} c-2^{\prime} c-T S$ & -866.099335 & 0.285558 & -865.813777 & 11.05 & 0.234230 & -865.865106 & 10.46 \\
\hline $2 ' c$ & -866.137331 & 0.287100 & -865.850231 & -11.83 & 0.237033 & -865.900298 & -11.62 \\
\hline $1 ' d$ & -882.130329 & 0.275302 & -881.855027 & 0.00 & 0.225645 & -881.904684 & 0.00 \\
\hline $1^{\prime} d-2^{\prime} d-T S$ & -882.112545 & 0.274438 & -881.838107 & 10.62 & 0.224647 & -881.887898 & 10.53 \\
\hline $2 ' d$ & -882.148941 & 0.275959 & -881.872982 & -11.27 & 0.225610 & -881.923331 & -11.70 \\
\hline 1'e & -655.624327 & 0.196915 & -655.427411 & 0.00 & 0.154721 & -655.469606 & 0.00 \\
\hline 1'e-2'e-TS & -655.603201 & 0.196329 & -655.406873 & 12.89 & 0.156923 & -655.446279 & 14.64 \\
\hline 2'e & -655.641548 & 0.197508 & -655.444040 & -23.32 & 0.155254 & -655.486294 & -10.47 \\
\hline 1'f & -978.226053 & 0.194336 & -978.031717 & 0.00 & 0.150467 & -978.075587 & 0.00 \\
\hline 1'f-2'f-TS & -978.211335 & 0.193990 & -978.017345 & 9.02 & 0.151758 & -978.059577 & 10.05 \\
\hline $2 ' f$ & -978.253087 & 0.195647 & -978.057440 & -16.14 & 0.152078 & -978.101009 & -15.95 \\
\hline 1'g & -580.533182 & 0.190905 & -580.342277 & 0.00 & 0.149842 & -580.383340 & 0.00 \\
\hline 1'g-2'g-TS & -580.520784 & 0.189939 & -580.330845 & 7.17 & 0.148772 & -580.372012 & 7.11 \\
\hline 2'g & -580.571960 & 0.191490 & -580.380470 & -23.97 & 0.149785 & -580.422175 & -24.37 \\
\hline $1 ' h$ & -655.598518 & 0.196781 & -655.401737 & 0.00 & 0.154272 & -655.444246 & 0.00 \\
\hline 1'h-2'h-TS & -655.571831 & 0.195375 & -655.376457 & 15.86 & 0.152264 & -655.419567 & 15.49 \\
\hline 2'h & -655.610164 & 0.196669 & -655.413494 & -7.38 & 0.153955 & -655.456208 & -7.51 \\
\hline $1 ' i$ & -900.151079 & 0.252729 & -899.898350 & 0.00 & 0.201329 & -899.949750 & 0.00 \\
\hline $2 ' i$ & -900.167734 & 0.252623 & -899.915111 & -10.52 & 0.201402 & -899.966332 & -10.41 \\
\hline 1 'j & -875.718305 & 0.288907 & -875.429398 & 0.00 & 0.240434 & -875.477870 & 0.00 \\
\hline 1'j-2'j-TS & -875.675623 & 0.287142 & -875.388481 & 25.68 & 0.238549 & -875.437074 & 25.60 \\
\hline 2 'j & -875.754536 & 0.289230 & -875.465306 & -22.53 & 0.241066 & -875.513469 & -22.34 \\
\hline 1'k & -950.804751 & 0.294386 & -950.510365 & 0.00 & 0.244277 & -950.560473 & 0.00 \\
\hline 1'k-2'k-TS & -950.766779 & 0.293305 & -950.473474 & 23.15 & 0.244174 & -950.522605 & 23.76 \\
\hline 2'k & -950.829768 & 0.294189 & -950.535579 & -15.82 & 0.244871 & -950.584896 & -15.33 \\
\hline
\end{tabular}


Table S4. Total energy values (E, RI-CCSD/cc-pVTZ//RI-BP86/TZVP level of approximation), and corrected energy values (E+ZPE and E+CP, a.u) for singlet and triplet state of carbenes $\mathbf{1}^{\prime} \mathbf{a}-\mathbf{1}^{\prime} \mathbf{k}$ using calculated at the RI-BP86/TZVP level of approximation zero-point energy correction (ZPE) and chemical potential (correction to Gibbs free energy, CP).

\begin{tabular}{|c|c|c|c|c|c|c|c|}
\hline Structure & E, a.u. & ZPE, a.u. & E+ZPE, a.u. & $\Delta \mathrm{E}, \mathrm{kcal} \cdot \mathrm{mol}^{-1}$ & CP, a.u. & $E+C P$, a.u. & $\Delta \mathrm{G}, \mathrm{kcal} \cdot \mathrm{mol}^{-1}$ \\
\hline 1 'a & -674.939763 & 0.235835 & -674.703928 & 0.00 & 0.190947 & -674.748816 & 0.00 \\
\hline 1'a-T & -674.873439 & 0.233898 & -674.639541 & 40.63 & 0.188783 & -674.684656 & 40.51 \\
\hline 1'b & -905.306880 & 0.314845 & -904.992035 & 0.00 & 0.262422 & -905.044458 & 0.00 \\
\hline 1'b-T & -905.234060 & 0.312532 & -904.921528 & 44.44 & 0.258793 & -904.975267 & 43.59 \\
\hline $1 ' c$ & -866.117869 & 0.286491 & -865.831379 & 0.00 & 0.236088 & -865.881781 & 0.00 \\
\hline $1^{\prime} c-T$ & -866.044065 & 0.285006 & -865.759059 & 45.53 & 0.233773 & -865.810292 & 45.04 \\
\hline 1'd & -882.130329 & 0.275302 & -881.855027 & 0.00 & 0.225645 & -881.904684 & 0.00 \\
\hline $1^{\prime} d-T$ & -882.059516 & 0.273662 & -881.785854 & 43.58 & 0.223135 & -881.836381 & 43.03 \\
\hline 1'e & -655.624327 & 0.196915 & -655.427411 & 0.00 & 0.154721 & -655.469606 & 0.00 \\
\hline 1'e-T & -655.535858 & 0.194634 & -655.341223 & 54.32 & 0.150444 & -655.385414 & 53.08 \\
\hline 1'f & -978.226053 & 0.194336 & -978.031717 & 0.00 & 0.150467 & -978.075587 & 0.00 \\
\hline $1^{\prime} f-T$ & -978.161031 & 0.192370 & -977.968661 & 39.86 & 0.146692 & -978.014339 & 38.79 \\
\hline 1'g & -580.533182 & 0.190905 & -580.342277 & 0.00 & 0.149842 & -580.383340 & 0.00 \\
\hline 1'g-T & -580.486102 & 0.189693 & -580.296408 & 29.14 & 0.148368 & -580.337734 & 28.12 \\
\hline 1'h & -655.598518 & 0.196781 & -655.401737 & 0.00 & 0.154272 & -655.444246 & 0.00 \\
\hline 1'h-T & -655.511782 & 0.194850 & -655.316931 & 53.45 & 0.151148 & -655.360633 & 52.70 \\
\hline $1 ' i$ & -900.151079 & 0.252729 & -899.898350 & 0.00 & 0.201329 & -899.949750 & 0.00 \\
\hline 1'i-T & -900.081429 & 0.251130 & -899.830299 & 42.69 & 0.198240 & -899.883188 & 42.02 \\
\hline $1 ' j$ & -875.718305 & 0.288907 & -875.429398 & 0.00 & 0.240434 & -875.477870 & 0.00 \\
\hline 1'j-T & -875.650941 & 0.286975 & -875.363967 & 41.10 & 0.237307 & -875.413634 & 40.34 \\
\hline 1'k & -950.804751 & 0.294386 & -950.510365 & 0.00 & 0.244277 & -950.560473 & 0.00 \\
\hline 1'k-T & -950.736966 & 0.292717 & -950.444249 & 41.33 & 0.242072 & -950.494894 & 40.93 \\
\hline
\end{tabular}



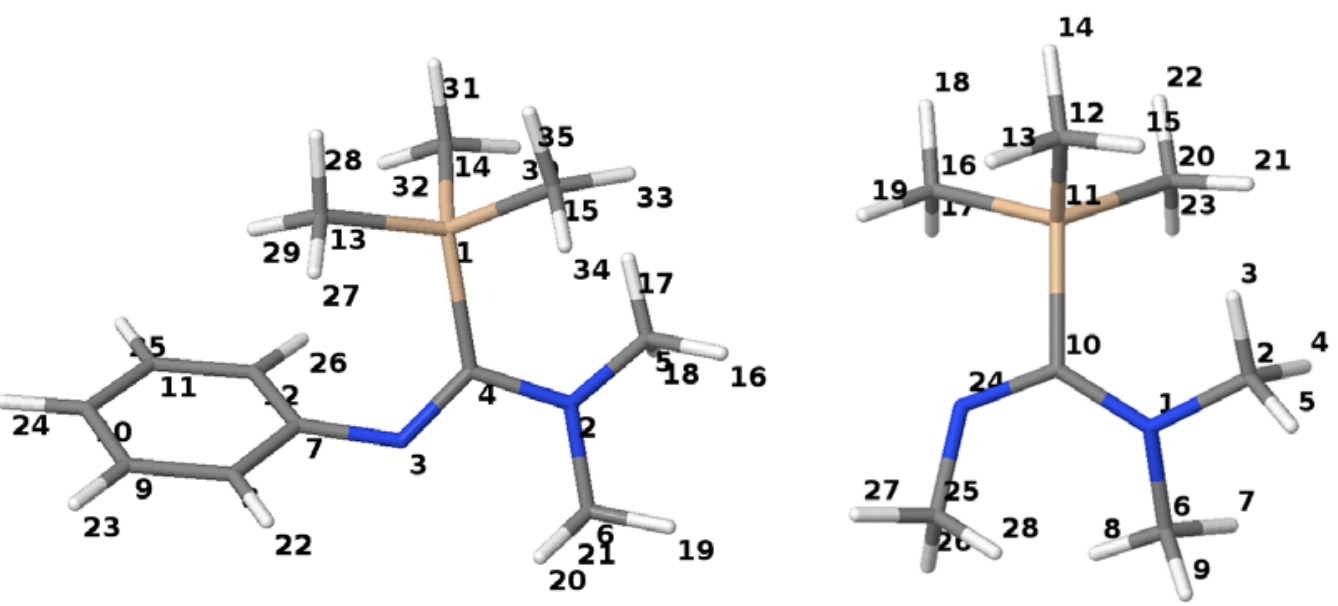

Fig. S1. Atom numbering for 2'c (left) and 2'a (right).

We have employed delocalization and localization indices (DIs and LIS) for the analysis of the electron distribution in the compounds of interest. As can be seen from Table S5, DI between MeN and Si atoms (highlighted in green) reaches maximum for 2'a-E, which has LP of nitrogen atom in the position unfavorable for supposed hyperconjugation. Additionally, three-center indices for MeN-C-Si moiety do not support hypothesis of hyperconjugation, as the largest index is observed for 2'a-E. Therefore, elongation of C-Si bond in the compounds 2'c and 2'a has different nature from LP $\rightarrow \sigma^{*}$ interaction.

Analysis of the difference of LIs between $Z$ and $E$ conformer shows that on changing conformation electron depletion occurs for carbon and hydrogens of NMe groups, and C-Si moiety is enriched in electrons (Table S6, Fig. 4). This may lead to the observed bond elongation. Total LI difference for hydrogen atoms of -0.0120 shows that $Z \rightarrow E$ conformational change leads to the electron transfer to heavy atoms, thus stabilizing $Z$ conformation (see also ${ }^{[1]}$ ).

Table S5. Delocalization indices for the compounds 2'c and 2'a (HF/SVP//RI-BP86/TZVP)

\begin{tabular}{|c|c|c|c|c|}
\hline Atom (number) & \multicolumn{3}{|c|}{ Delocalization indices } & \multirow{2}{*}{$\begin{array}{c}\text { Multicenter index } \\
\text { (MeN-C-Si) } \\
0.0320 \\
\end{array}$} \\
\hline $2 ' c$ & $\mathrm{~N}-\mathrm{Me}(3)$ & $C(4)$ & $\mathrm{Si}(1)$ & \\
\hline \multicolumn{5}{|l|}{$\mathrm{N}-\mathrm{Me}(3)$} \\
\hline$C(4)$ & 1.7500 & & & \\
\hline Si (1) & 0.1211 & 0.9363 & & \\
\hline $\mathrm{NMe}_{2}(2)$ & 0.1637 & 1.2594 & 0.0844 & \\
\hline 2'a-E & $\mathrm{N}-\mathrm{Me}(24)$ & $\mathrm{C}(10)$ & Si (11) & 0.0472 \\
\hline \multicolumn{5}{|l|}{$\mathrm{N}-\mathrm{Me}(24)$} \\
\hline C (10) & 1.8162 & & & \\
\hline Si (11) & 0.1945 & 0.9543 & & \\
\hline $\mathrm{NMe}_{2}(1)$ & 0.1418 & 1.2350 & 0.0757 & \\
\hline 2’a-Z & N-Me (24) & $C(10)$ & Si (11) & 0.0339 \\
\hline \multicolumn{5}{|l|}{$\mathrm{N}-\mathrm{Me}(24)$} \\
\hline C (10) & 1.8347 & & & \\
\hline Si (11) & 0.1267 & 0.9450 & & \\
\hline $\mathrm{NMe}_{2}(1)$ & 0.1568 & 1.2056 & 0.0927 & \\
\hline
\end{tabular}

[1] D. Ferro-Costas, A. Vila, R. A. Mosquera, J. Phys. Chem. A 2013, 117, 1641-1650. 
Table S6. Localization indices for the E and Z conformations of the compound 2'a and their difference

(HF/SVP//RI-BP86/TZVP)

\begin{tabular}{|c|c|c|c|c|c|c|}
\hline \multicolumn{7}{|c|}{ 2'a-E } \\
\hline $1(\mathrm{~N})$ & 5.3060 & 2(C) & \begin{tabular}{|l|l|l|}
4.2890 & $3(\mathrm{H})$
\end{tabular} & 0.2850 & $4(\mathrm{H})$ & 0.3090 \\
\hline $5(H)$ & 0.3100 & $6(C)$ & \begin{tabular}{|l|l|l}
4.2980 & $(H)$
\end{tabular} & 0.3120 & $8(\mathrm{H})$ & 0.2810 \\
\hline $9(H)$ & 0.3120 & 10 (C) & \begin{tabular}{l|l|l}
3.5980 & $11(\mathrm{Si})$
\end{tabular} & 11.8050 & $12(\mathrm{C})$ & 4.4000 \\
\hline $13(\mathrm{H})$ & 0.3020 & $14(\mathrm{H})$ & \begin{tabular}{l|l|}
0.3100 & $15(\mathrm{H})$
\end{tabular} & 0.3080 & $16(C)$ & 4.3860 \\
\hline $17(H)$ & 0.3040 & $18(H)$ & \begin{tabular}{l|l|l}
0.3150 & $19(\mathrm{H})$
\end{tabular} & 0.2890 & $20(C)$ & 4.4060 \\
\hline $21(H)$ & 0.3020 & $22(\mathrm{H})$ & \begin{tabular}{|l|l|}
0.3090 & $23(\mathrm{H})$
\end{tabular} & 0.3050 & $24(N)$ & 5.5840 \\
\hline $25(C)$ & 4.2980 & $26(\mathrm{H})$ & \begin{tabular}{|l|l|}
0.3100 & $27(\mathrm{H})$
\end{tabular} & 0.3150 & $28(\mathrm{H})$ & 0.2950 \\
\hline \multicolumn{7}{|c|}{ 2'a-z } \\
\hline $1(\mathrm{~N})$ & 5.3170 & 2(C) & $\begin{array}{ll}4.2920 & 3(\mathrm{H}) \\
\end{array}$ & 0.2810 & $4(\mathrm{H})$ & 0.3090 \\
\hline $5(H)$ & 0.3140 & 6(C) & $4.2830 \mid 7(\mathrm{H})$ & 0.3140 & $8(\mathrm{H})$ & 0.2850 \\
\hline $9(\mathrm{H})$ & 0.3060 & $10(C)$ & \begin{tabular}{|l|l|}
3.6130 & $11(\mathrm{Si})$
\end{tabular} & 11.8220 & 12(C) & 4.4000 \\
\hline $13(H)$ & 0.3020 & $14(\mathrm{H})$ & $\begin{array}{lll}0.3080 & 15(\mathrm{H})\end{array}$ & 0.3060 & $16(C)$ & 4.4030 \\
\hline $17(H)$ & 0.3010 & $18(\mathrm{H})$ & 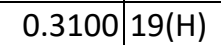 & 0.2970 & $20(C)$ & 4.4050 \\
\hline $21(\mathrm{H})$ & 0.3040 & $22(\mathrm{H})$ & \begin{tabular}{l|l|}
0.3080 & $23(\mathrm{H})$ \\
\end{tabular} & 0.2940 & $24(N)$ & 5.5810 \\
\hline $25(C)$ & 4.2860 & 26(H) & \begin{tabular}{l|l|}
0.3140 & $27(\mathrm{H})$
\end{tabular} & 0.3090 & $28(\mathrm{H})$ & 0.2990 \\
\hline \multicolumn{7}{|c|}{ Difference } \\
\hline $1(\mathrm{~N})$ & 0.0110 & 2(C) & \begin{tabular}{l|l|}
0.0030 & $3(\mathrm{H})$ \\
\end{tabular} & -0.0040 & $4(\mathrm{H})$ & 0.0000 \\
\hline $5(\mathrm{H})$ & 0.0040 & $6(C)$ & \begin{tabular}{ll|l}
-0.0150 & $7(\mathrm{H})$ \\
\end{tabular} & 0.0020 & $8(\mathrm{H})$ & 0.0040 \\
\hline $9(\mathrm{H})$ & -0.0060 & $10(C)$ & \begin{tabular}{|l|l}
0.0150 & $11(\mathrm{Si})$
\end{tabular} & 0.0170 & $12(C)$ & 0.0000 \\
\hline $13(\mathrm{H})$ & 0.0000 & 14(H) & \begin{tabular}{l|l}
-0.0020 & $15(\mathrm{H})$ \\
\end{tabular} & -0.0020 & $16(C)$ & 0.0170 \\
\hline 17(H) & -0.0030 & $18(\mathrm{H})$ & \begin{tabular}{l|l|}
-0.0050 & $19(\mathrm{H})$
\end{tabular} & 0.0080 & $20(C)$ & -0.0010 \\
\hline $21(\mathrm{H})$ & 0.0020 & $22(\mathrm{H})$ & \begin{tabular}{l|l}
-0.0010 & $23(\mathrm{H})$ \\
\end{tabular} & -0.0110 & $24(N)$ & -0.0030 \\
\hline $25(C)$ & -0.0120 & 26(H) & \begin{tabular}{|l|l|}
0.0040 & $27(\mathrm{H})$ \\
\end{tabular} & -0.0060 & $28(\mathrm{H})$ & 0.0040 \\
\hline
\end{tabular}


Cartesian coordinates for the equilibrium structures 1a-1k (RI-BP86/TZVP).

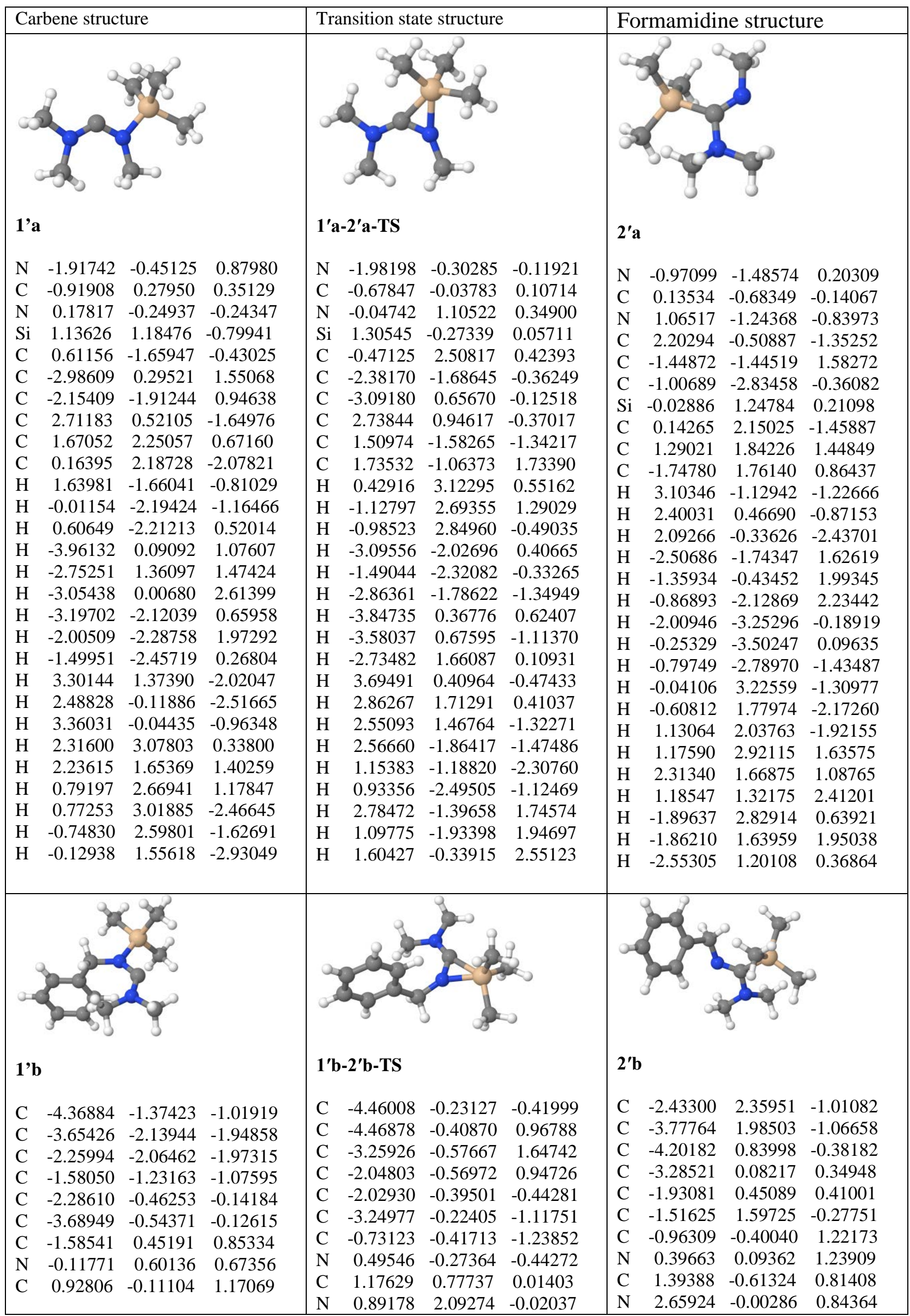




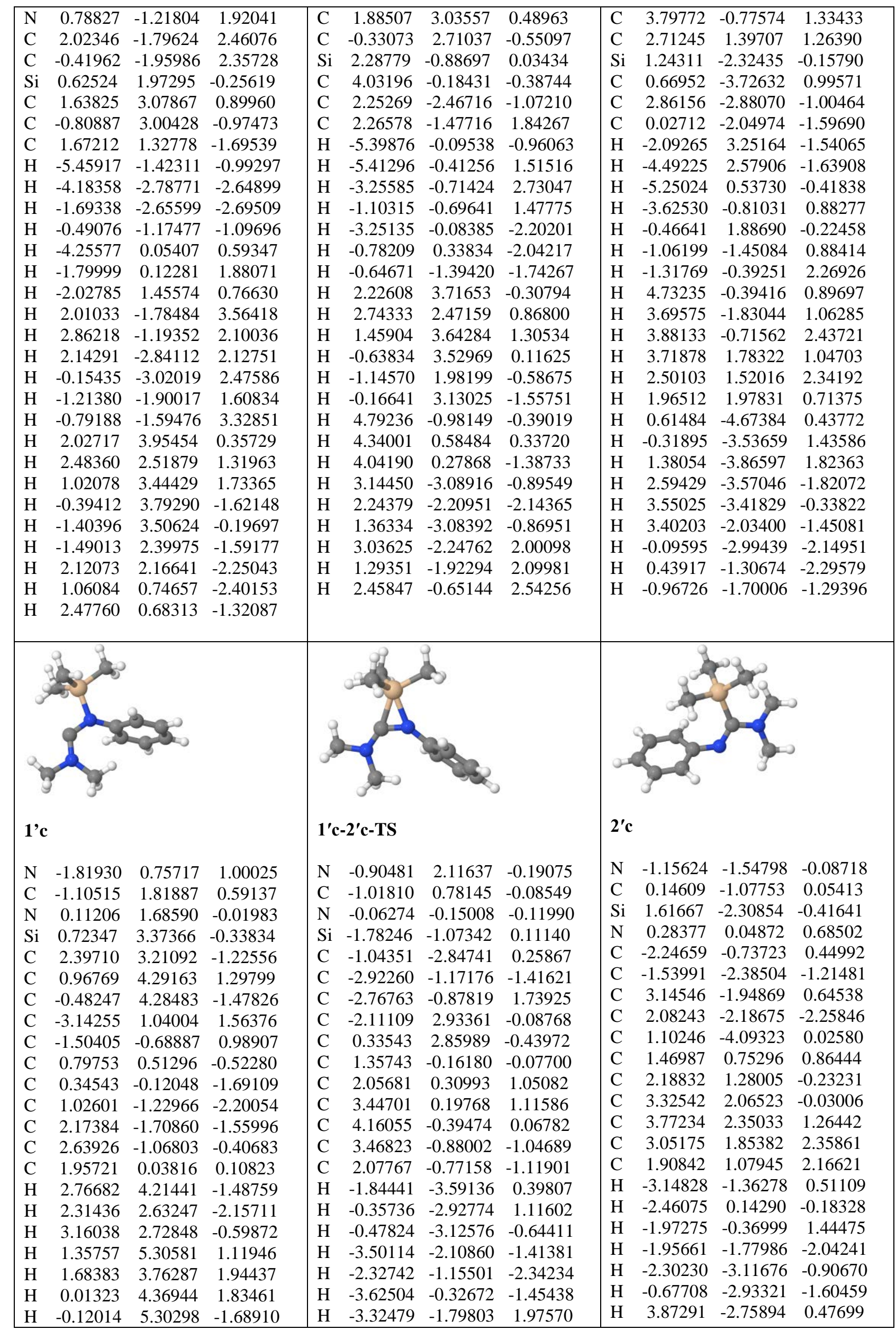




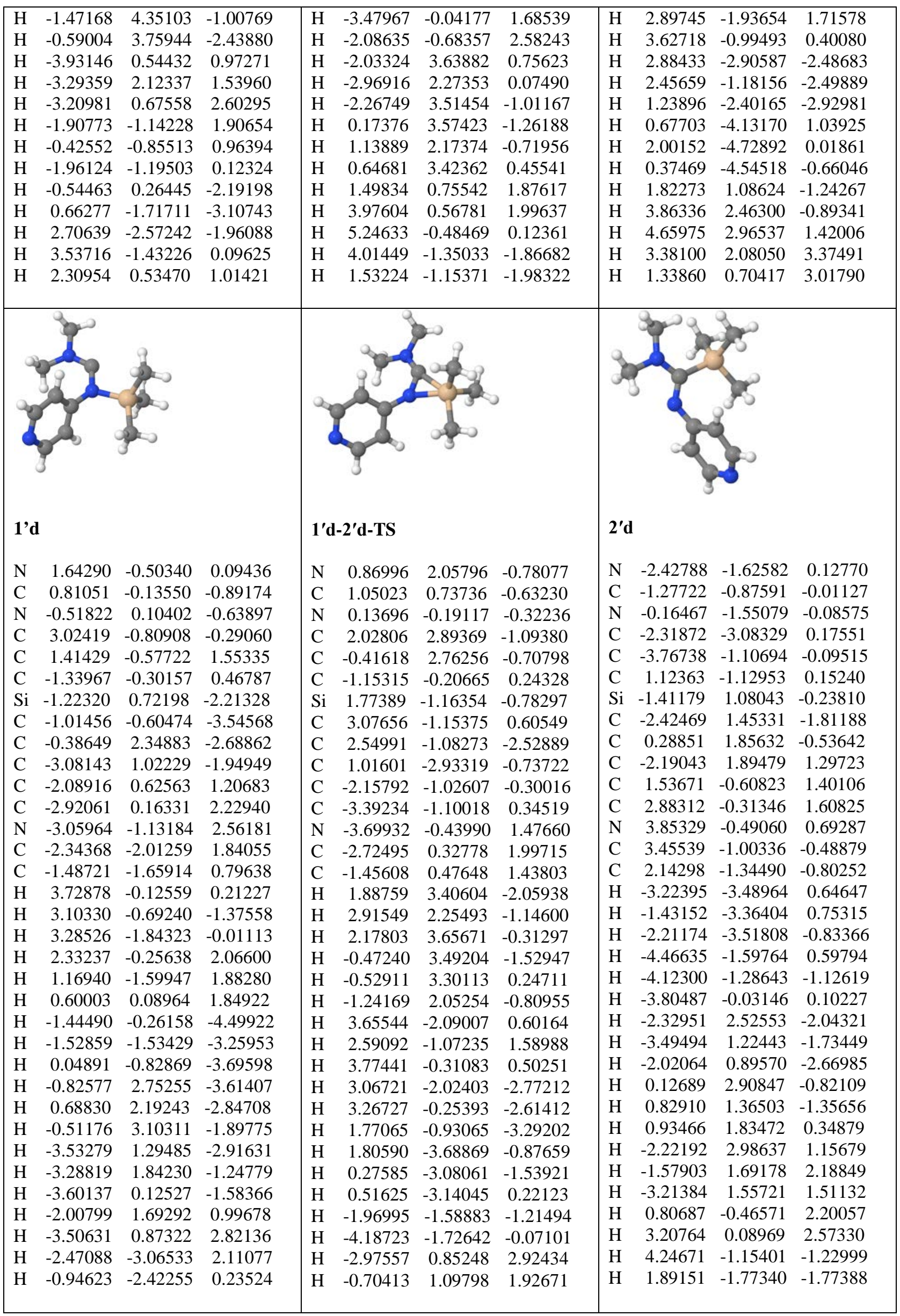




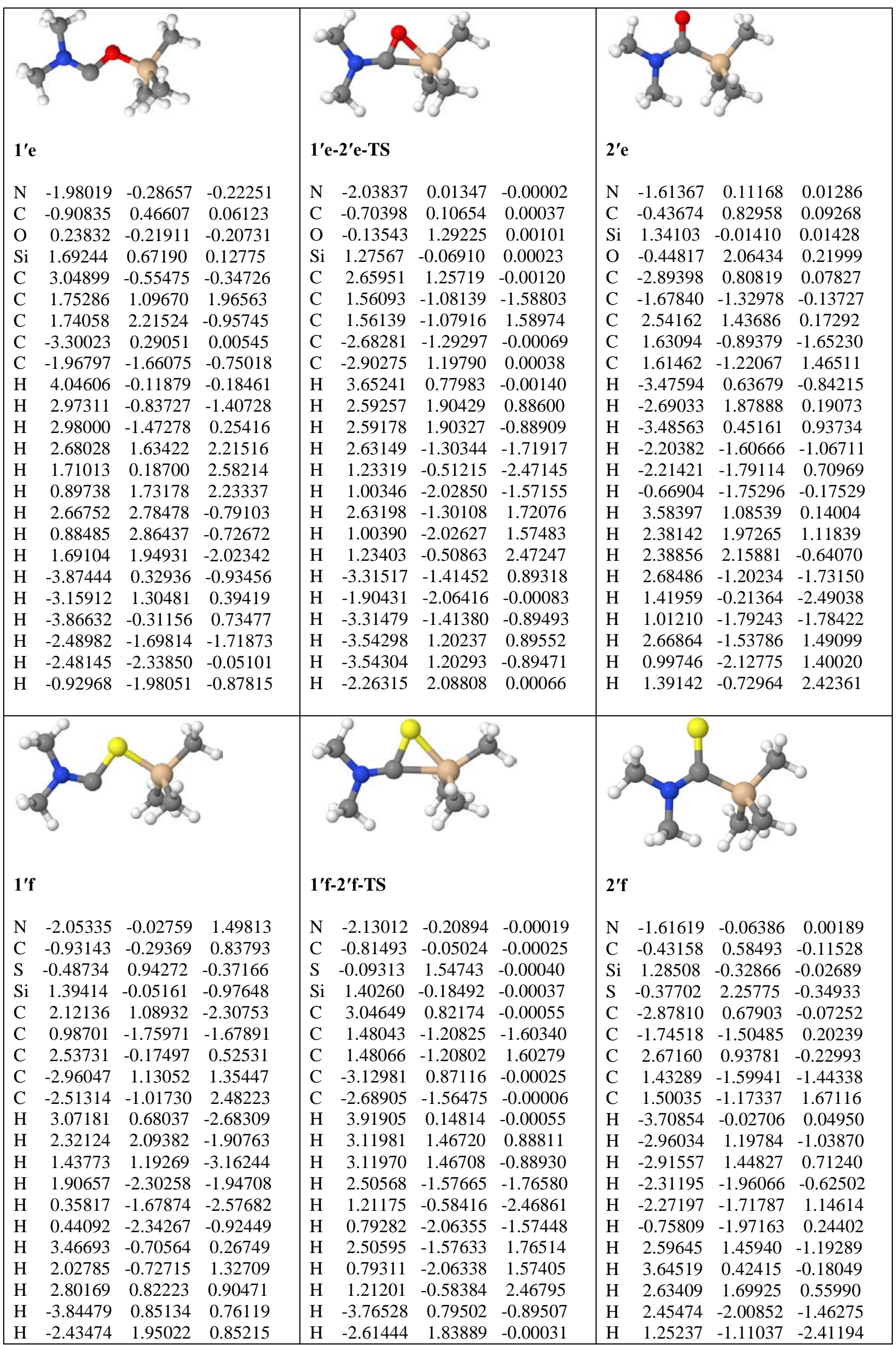




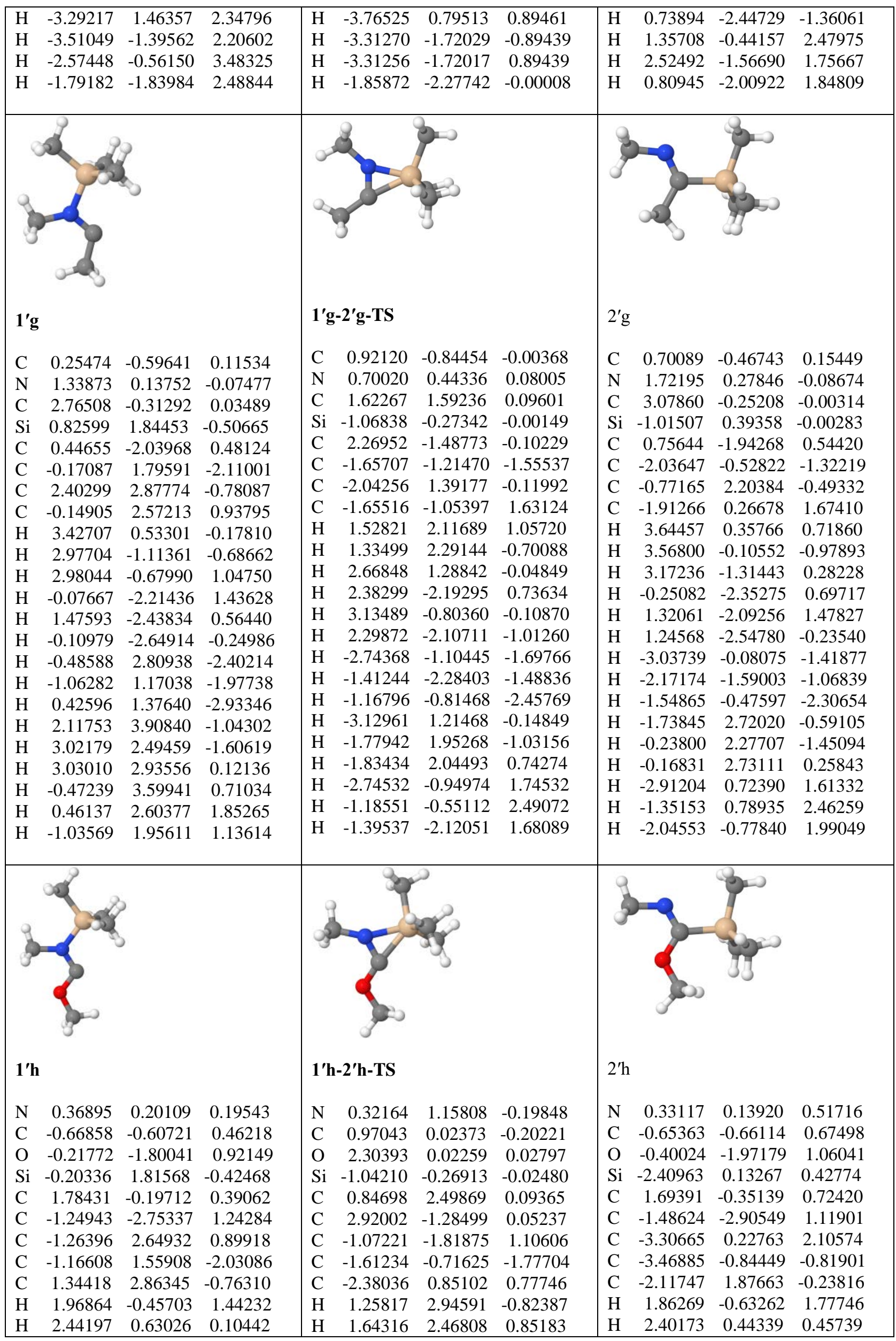




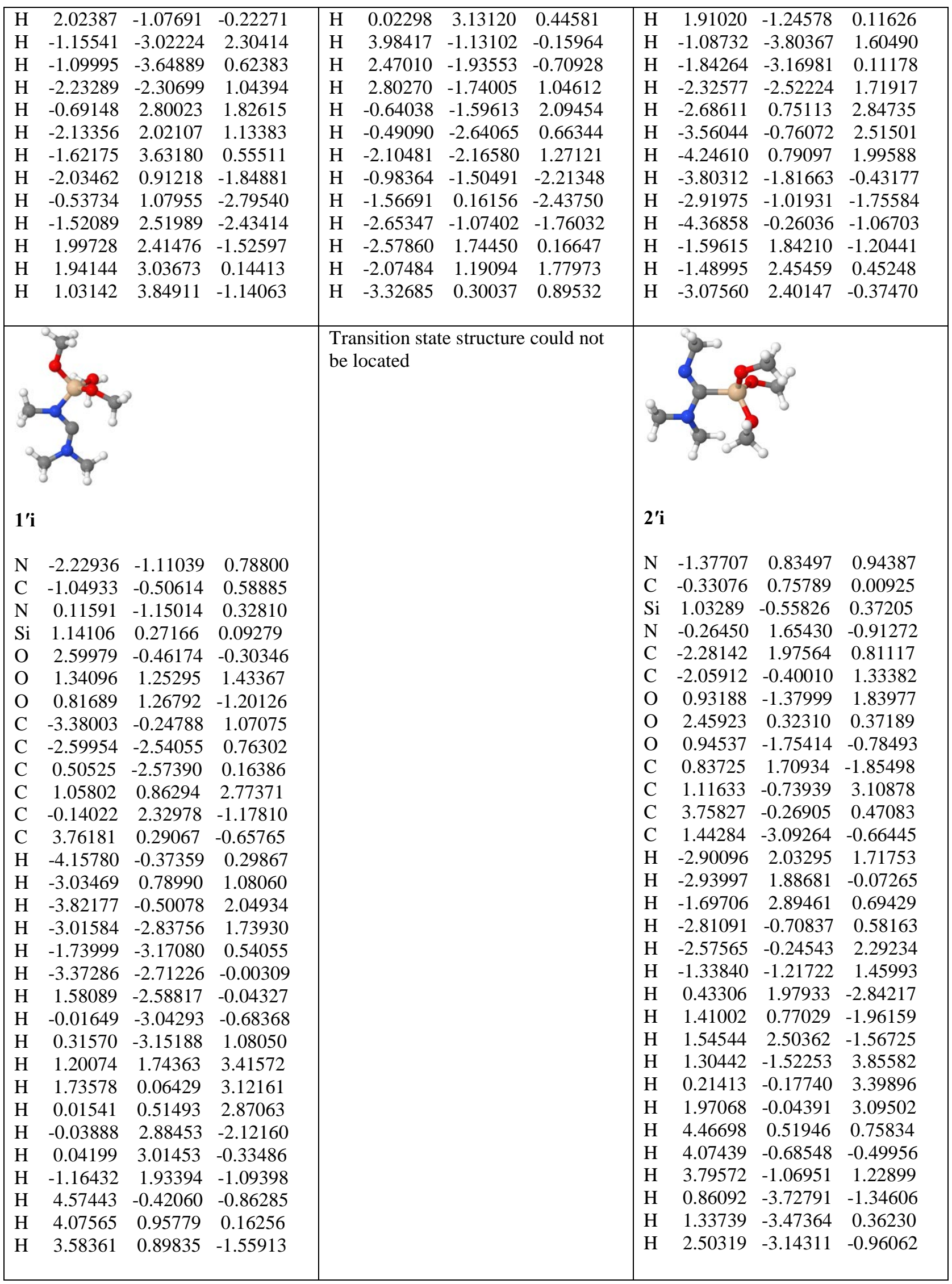




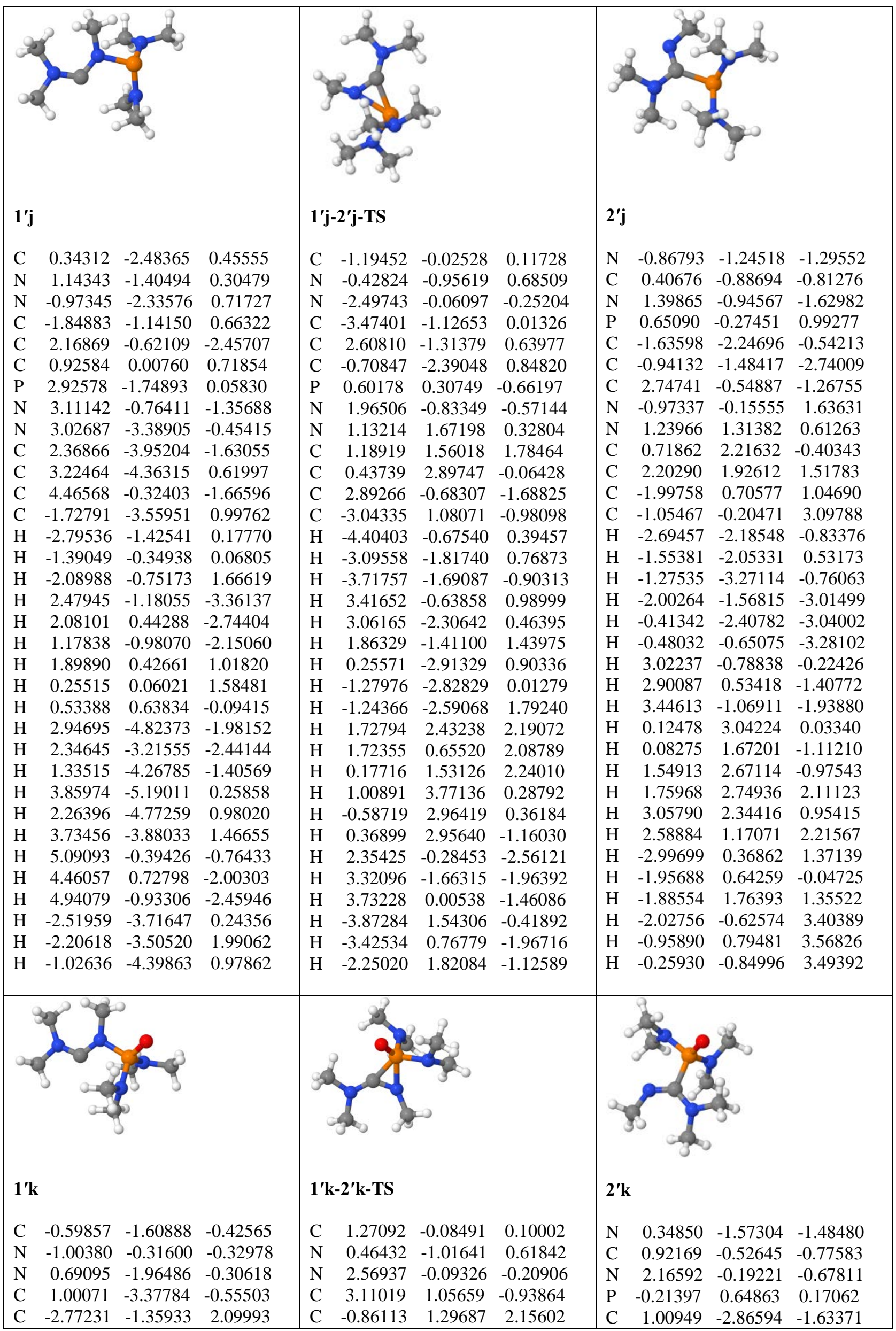




\begin{tabular}{|c|c|c|c|c|c|c|c|c|c|c|c|}
\hline C & -0.24313 & 0.96089 & -0.18460 & C & 0.64437 & -2.46082 & 0.82223 & C & -1.04871 & -1.55935 & -1.91700 \\
\hline $\mathrm{P}$ & -2.75954 & -0.11387 & -0.41791 & $\mathrm{P}$ & -0.56075 & 0.25315 & -0.40081 & C & 3.19346 & -0.76145 & -1.53981 \\
\hline $\mathrm{N}$ & -3.30584 & -0.37711 & 1.16447 & $\mathrm{~N}$ & -1.04052 & 1.50413 & 0.72028 & $\mathrm{~N}$ & 0.73122 & 1.78534 & 1.01674 \\
\hline $\mathrm{N}$ & -3.42283 & -1.31046 & -1.41799 & $\mathrm{~N}$ & -1.92828 & -0.84483 & -0.48192 & $\mathrm{~N}$ & -0.96999 & -0.36377 & 1.31494 \\
\hline C & -3.70224 & -2.66588 & -0.95441 & C & -2.58972 & -1.25852 & 0.74976 & C & -0.35448 & -1.53378 & 1.92254 \\
\hline C & -3.08323 & -1.22723 & -2.83971 & C & -2.90067 & -0.59193 & -1.55014 & C & -2.13813 & 0.16552 & 2.01560 \\
\hline C & -4.56863 & 0.24392 & 1.56226 & C & -0.55909 & 2.83098 & 0.31724 & C & 1.62700 & 1.36735 & 2.09155 \\
\hline C & 1.91591 & -1.18828 & 0.00951 & C & 3.53473 & -1.15785 & 0.09441 & C & 1.19929 & 2.96276 & 0.27995 \\
\hline $\mathrm{O}$ & -3.07780 & 1.27655 & -0.92580 & $\mathrm{O}$ & -0.43149 & 0.76347 & -1.83584 & $\mathrm{O}$ & -1.19660 & 1.37320 & -0.73600 \\
\hline $\mathrm{H}$ & 1.69750 & -3.47770 & -1.40458 & $\mathrm{H}$ & 3.83355 & 1.60871 & -0.31651 & $\mathrm{H}$ & 0.34363 & -3.65831 & -1.24896 \\
\hline $\mathrm{H}$ & 0.06567 & -3.89463 & -0.78832 & $\mathrm{H}$ & 2.27868 & 1.70883 & -1.22370 & $\mathrm{H}$ & 1.93871 & -2.89526 & -1.05744 \\
\hline $\mathrm{H}$ & 1.47093 & -3.83521 & 0.33192 & $\mathrm{H}$ & 3.61888 & 0.71332 & -1.85281 & $\mathrm{H}$ & 1.23851 & -3.09913 & -2.68759 \\
\hline $\mathrm{H}$ & -3.50985 & -2.15585 & 2.31506 & $\mathrm{H}$ & -1.39828 & 2.09201 & 2.69696 & $\mathrm{H}$ & -1.65937 & -2.26167 & -1.32209 \\
\hline $\mathrm{H}$ & -2.51299 & -0.87317 & 3.05701 & $\mathrm{H}$ & 0.20552 & 1.33662 & 2.46214 & $\mathrm{H}$ & -1.10278 & -1.86673 & -2.97466 \\
\hline $\mathrm{H}$ & -1.87547 & -1.83279 & 1.68292 & $\mathrm{H}$ & -1.27265 & 0.33382 & 2.47364 & $\mathrm{H}$ & -1.46281 & -0.54875 & -1.82335 \\
\hline $\mathrm{H}$ & -0.93386 & 1.77405 & -0.43795 & $\mathrm{H}$ & -0.34047 & -2.93474 & 0.73108 & $\mathrm{H}$ & 2.81132 & -1.14536 & -2.50226 \\
\hline $\mathrm{H}$ & 0.59850 & 0.98371 & -0.88712 & $\mathrm{H}$ & 1.03760 & -2.67121 & 1.82986 & $\mathrm{H}$ & 3.74548 & -1.57576 & -1.03923 \\
\hline $\mathrm{H}$ & 0.11786 & 1.10929 & 0.84398 & $\mathrm{H}$ & 1.30789 & -2.91260 & 0.07013 & $\mathrm{H}$ & 3.93076 & 0.02758 & -1.74864 \\
\hline $\mathrm{H}$ & -4.52996 & -3.07873 & -1.55441 & $\mathrm{H}$ & -3.26701 & -0.47843 & 1.15507 & $\mathrm{H}$ & -0.05462 & -1.35159 & 2.97276 \\
\hline $\mathrm{H}$ & -4.02192 & -2.64861 & 0.09387 & $\mathrm{H}$ & -1.85529 & -1.50842 & 1.52581 & $\mathrm{H}$ & 0.54192 & -1.83025 & 1.36358 \\
\hline $\mathrm{H}$ & -2.81706 & -3.31960 & -1.04192 & $\mathrm{H}$ & -3.19622 & -2.15966 & 0.55622 & $\mathrm{H}$ & -1.06151 & -2.38187 & 1.91900 \\
\hline $\mathrm{H}$ & -3.91254 & -1.64315 & -3.43364 & $\mathrm{H}$ & -3.62708 & 0.20203 & -1.28197 & $\mathrm{H}$ & -1.88858 & 0.51833 & 3.03361 \\
\hline $\mathrm{H}$ & -2.16108 & -1.79216 & -3.07365 & $\mathrm{H}$ & -3.46490 & -1.51987 & -1.74465 & $\mathrm{H}$ & -2.90998 & -0.61786 & 2.09913 \\
\hline $\mathrm{H}$ & -2.94997 & -0.17633 & -3.12427 & $\mathrm{H}$ & -2.37088 & -0.28928 & -2.45780 & $\mathrm{H}$ & -2.55795 & 1.00151 & 1.44204 \\
\hline $\mathrm{H}$ & -4.83679 & 1.02300 & 0.83901 & $\mathrm{H}$ & -0.67316 & 2.95205 & -0.76552 & $\mathrm{H}$ & 2.60800 & 1.03732 & 1.70621 \\
\hline $\mathrm{H}$ & -4.45598 & 0.71419 & 2.55376 & $\mathrm{H}$ & 0.50644 & 2.99616 & 0.58404 & $\mathrm{H}$ & 1.18004 & 0.54458 & 2.66341 \\
\hline $\mathrm{H}$ & -5.39008 & -0.49436 & 1.62086 & $\mathrm{H}$ & -1.16239 & 3.59553 & 0.83165 & $\mathrm{H}$ & 1.77444 & 2.21302 & 2.78276 \\
\hline $\mathrm{H}$ & 2.48413 & -0.94854 & -0.90401 & $\mathrm{H}$ & 4.45008 & -0.70647 & 0.50648 & $\mathrm{H}$ & 2.19581 & 2.79716 & -0.16741 \\
\hline $\mathrm{H}$ & 2.55977 & -1.80097 & 0.65777 & $\mathrm{H}$ & 3.80463 & -1.72249 & -0.81279 & $\mathrm{H}$ & 1.25813 & 3.81998 & 0.97067 \\
\hline $\mathrm{H}$ & 1.67797 & -0.26730 & 0.54060 & $\mathrm{H}$ & 3.12528 & -1.84540 & 0.83909 & $\mathrm{H}$ & 0.47989 & 3.20250 & -0.51142 \\
\hline
\end{tabular}

\section{Cartesian coordinates and images for optimized (RI-BP86/TZVP) structures of triplete carbenes 1'}

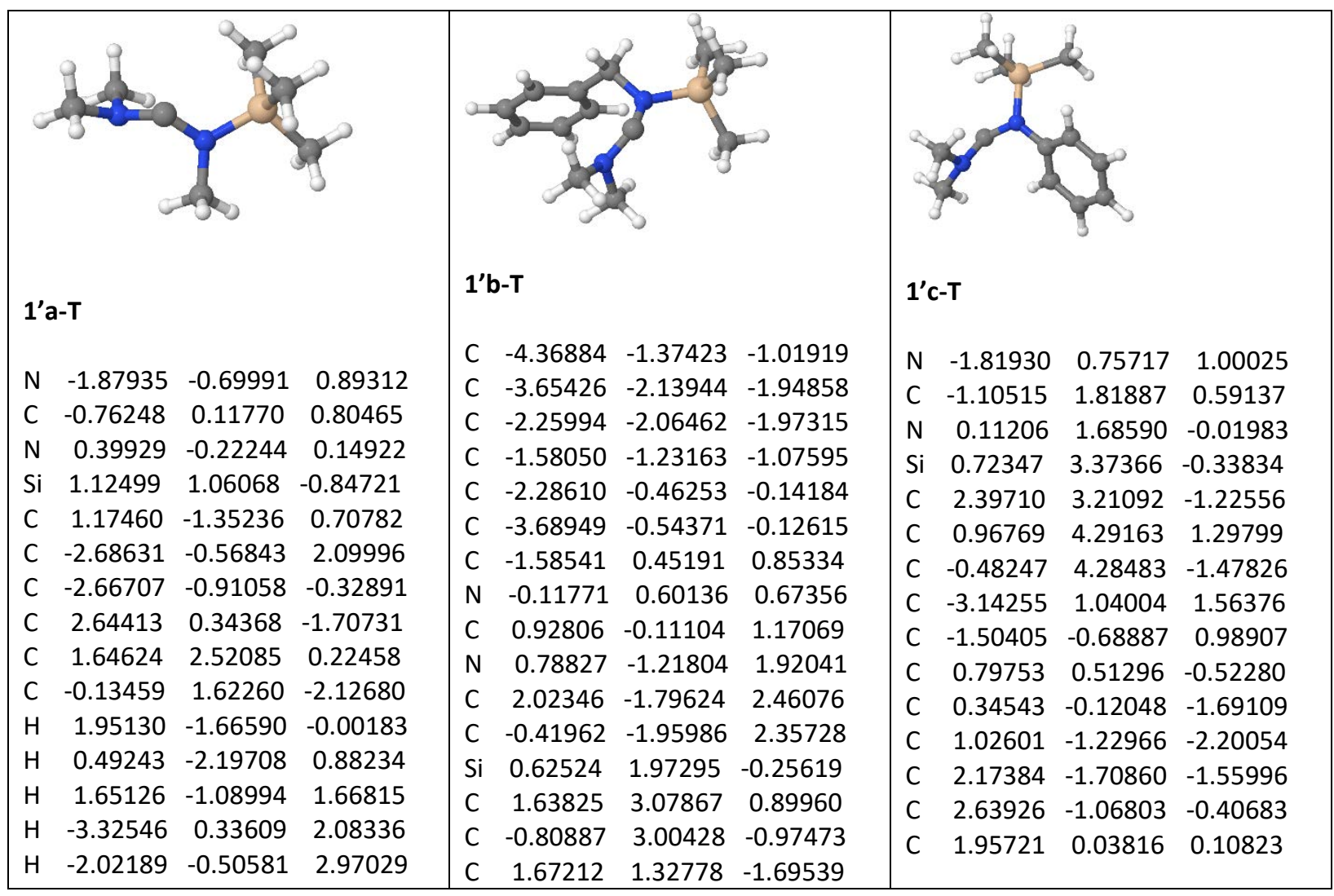




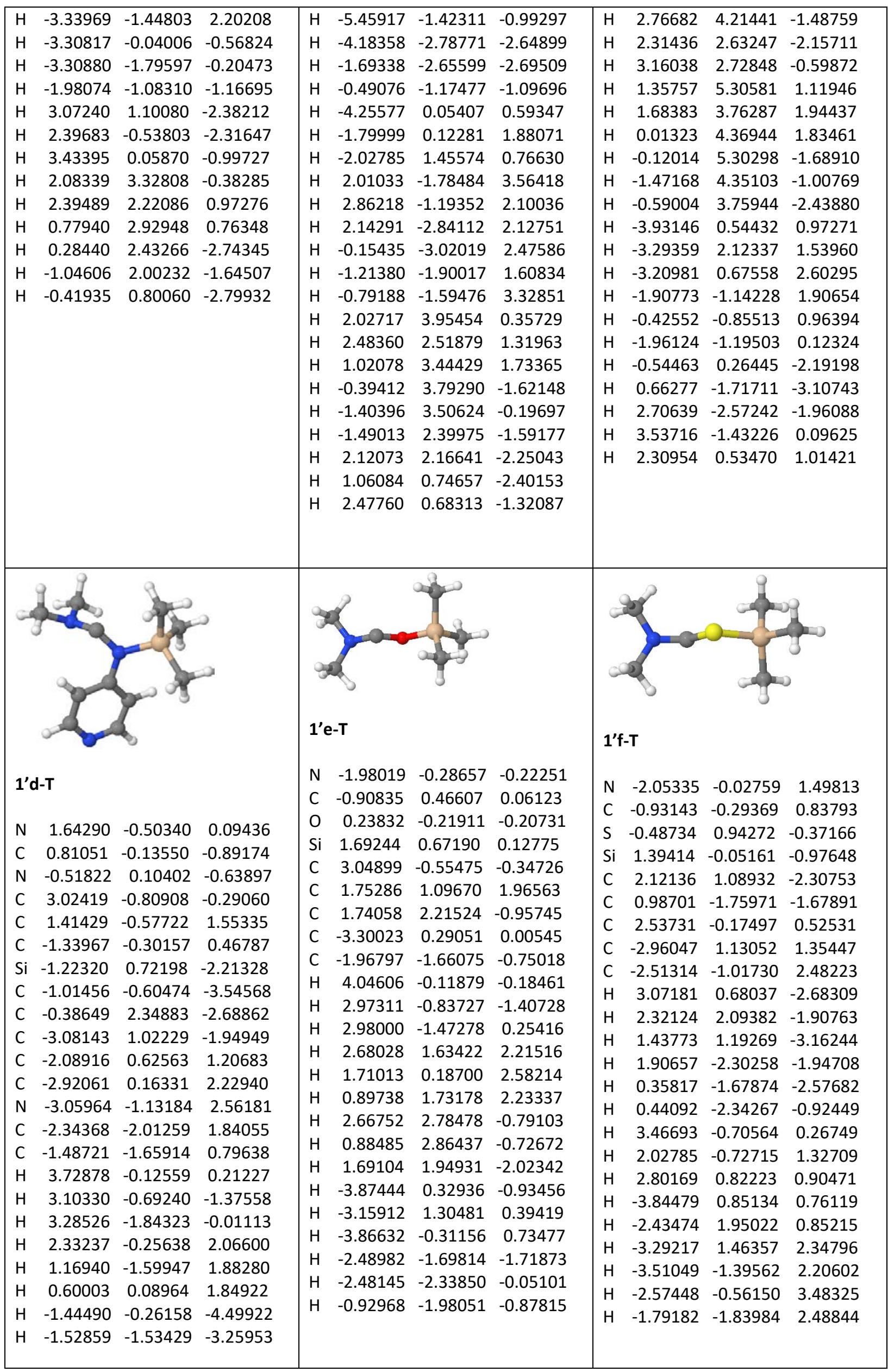




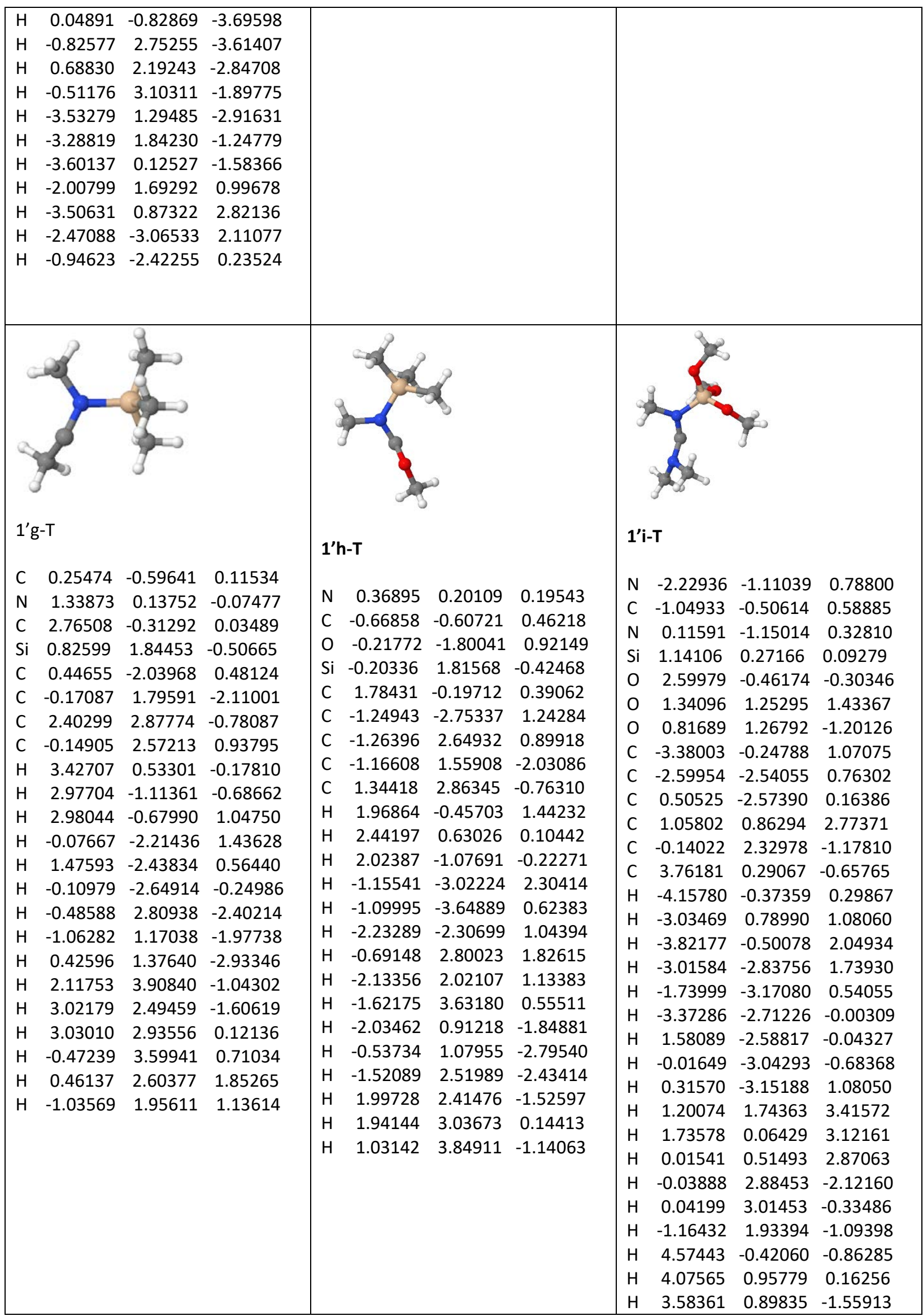




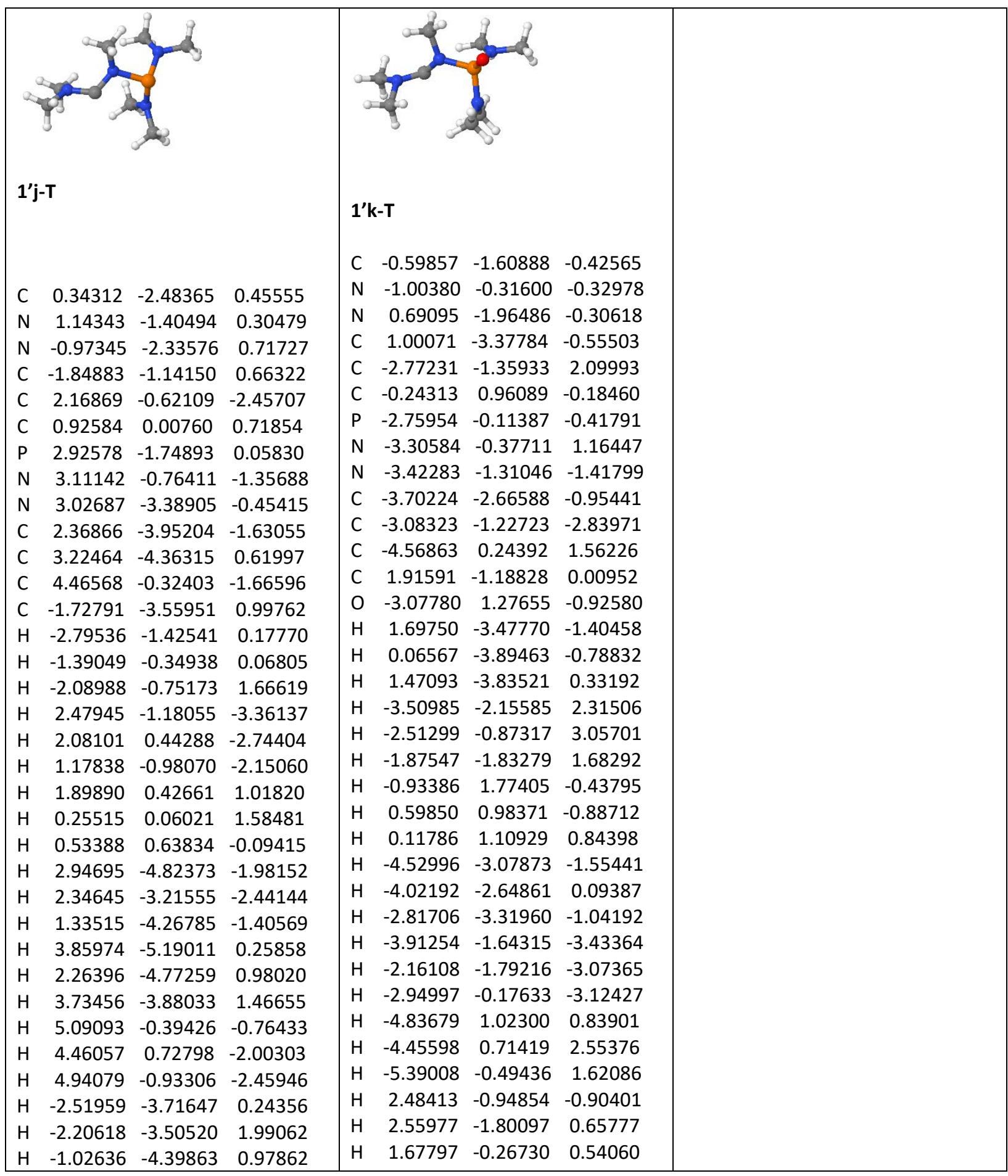

\section{Cartesian coordinates and image for optimized (RI-BP86/TZVP) structure of amidine 2'a-E}

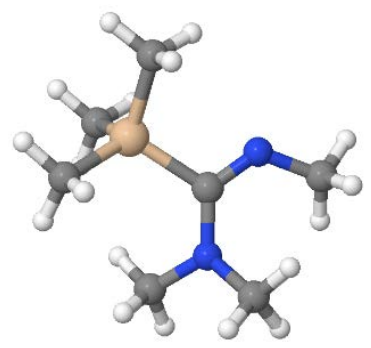

N $\quad-1.75381 \quad 0.07316 \quad 0.36355$ 


$\begin{array}{rrrr}\text { C } & -3.08350 & 0.66329 & 0.34475 \\ \text { H } & -3.14626 & 1.51396 & 1.04738 \\ \text { H } & -3.86781 & -0.06410 & 0.65081 \\ \text { H } & -3.33332 & 0.98321 & -0.68524 \\ \text { C } & -1.72189 & -1.32975 & 0.76006 \\ H & -2.19232 & -1.43702 & 1.76091 \\ \text { H } & -0.73695 & -1.77289 & 0.87423 \\ \text { H } & -2.29852 & -1.93812 & 0.03059 \\ \text { C } & -0.60200 & 0.89142 & -0.00495 \\ \text { Si } & -0.69785 & 2.76899 & -0.12702 \\ \text { C } & -1.94536 & 3.38690 & -1.37881 \\ \text { H } & -1.72960 & 2.93758 & -2.36980 \\ \text { H } & -1.86047 & 4.49045 & -1.45243 \\ \text { H } & -2.97925 & 3.14449 & -1.07012 \\ \text { C } & 0.97201 & 3.40597 & -0.68312 \\ \text { H } & 1.74914 & 3.09986 & 0.04700 \\ \text { H } & 0.94251 & 4.51308 & -0.74623 \\ \text { H } & 1.21533 & 2.98713 & -1.68124 \\ \text { C } & -1.04439 & 3.54262 & 1.54163 \\ \text { H } & -2.03818 & 3.24546 & 1.92573 \\ \text { H } & -1.01762 & 4.64700 & 1.44008 \\ \text { H } & -0.26316 & 3.22349 & 2.26144 \\ \text { N } & 0.57665 & 0.44776 & -0.32459 \\ \text { C } & 1.11817 & -0.88567 & -0.40001 \\ \text { H } & 1.27853 & -1.30030 & 0.61603 \\ \text { H } & 2.12690 & -0.82411 & -0.85709 \\ \text { H } & 0.49060 & -1.52946 & -1.05175\end{array}$




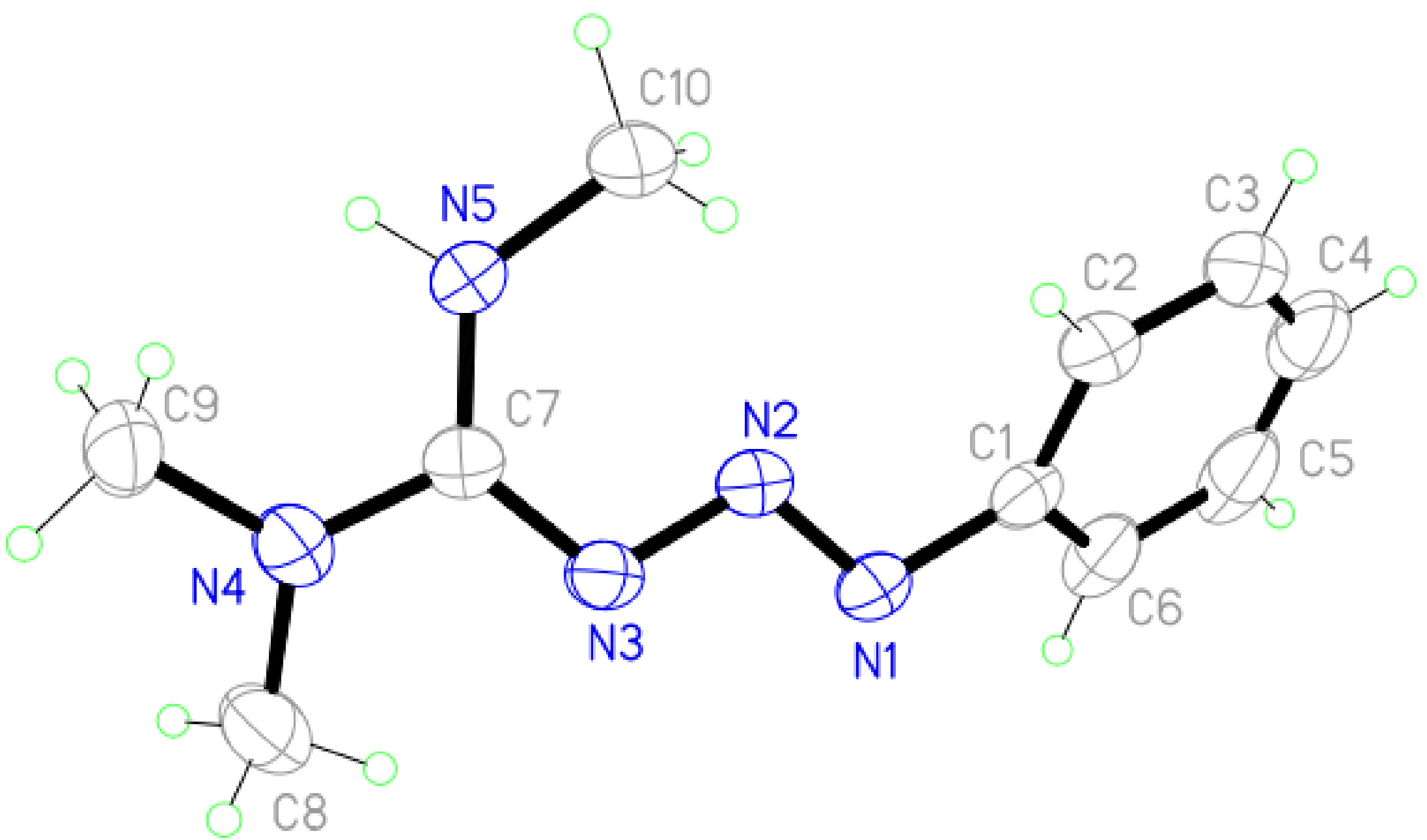

Figure S5. Molecular structure of compound $\mathbf{7}$ according to X-ray diffraction study. Thermal ellipsoids are shown with $50 \%$ probability level 
Crystal data adduct 7: $\mathrm{C}_{10} \mathrm{H}_{15} \mathrm{~N}_{5}, \mathrm{M}=205.27$, monoclinic, space group $P 2{ }_{1} / c, a=14.3044(5), b=6.7615(3), \quad c=$ 11.9313(4) $\AA, \beta=102.139(2)^{\circ}, \mathrm{V}=1128.18(7) \AA^{3}, \mathrm{Z}=4, \mathrm{~d}_{\mathrm{c}}=1.209 \mathrm{~g} \cdot \mathrm{cm}^{-3}, \mu=0.079 \mathrm{~mm}^{-1}, \mathrm{~F}(000)=440$, crystal size ca. $0.24 \times 0.26 \times 0.40 \mathrm{~mm}$. All crystallographic measurements were performed at ambient temperature on a Bruker Smart Apex II diffractometer operating in the $\omega$ scans mode. The intensity data were collected within the range of $2.9 \leq \theta \leq$ $26.34^{\circ}$ using $\mathrm{Mo}-\mathrm{K}_{\alpha}$ radiation $(\lambda=0.71078 \AA$ ). The intensities of 10949 reflections were collected (22982 unique reflections, $\mathrm{R}_{\mathrm{merg}}=0.0381$ ). The structure were solved by direct methods and refined by the full-matrix least-squares technique in the anisotropic approximation for non-hydrogen atoms using the Bruker SHELXTL program package. ${ }^{1}$ All $\mathrm{CH}$ hydrogen atoms were placed at calculated positions and refined as 'riding' model. Convergence was obtained at R1 = 0.0490 and $w R 2=0.11199$ for 1704 observed reflections with $\mathrm{I} \geq 2 \sigma(\mathrm{I}), \mathrm{R} 1=0.0690$ and $\mathrm{wR} 2=0.1323, \mathrm{GOF}=1.023$ for 2298 independent reflections, 143 parameters, the largest and minimal peaks in the final difference map 0.20 and $0.17 \mathrm{e} / \AA^{3}$. 


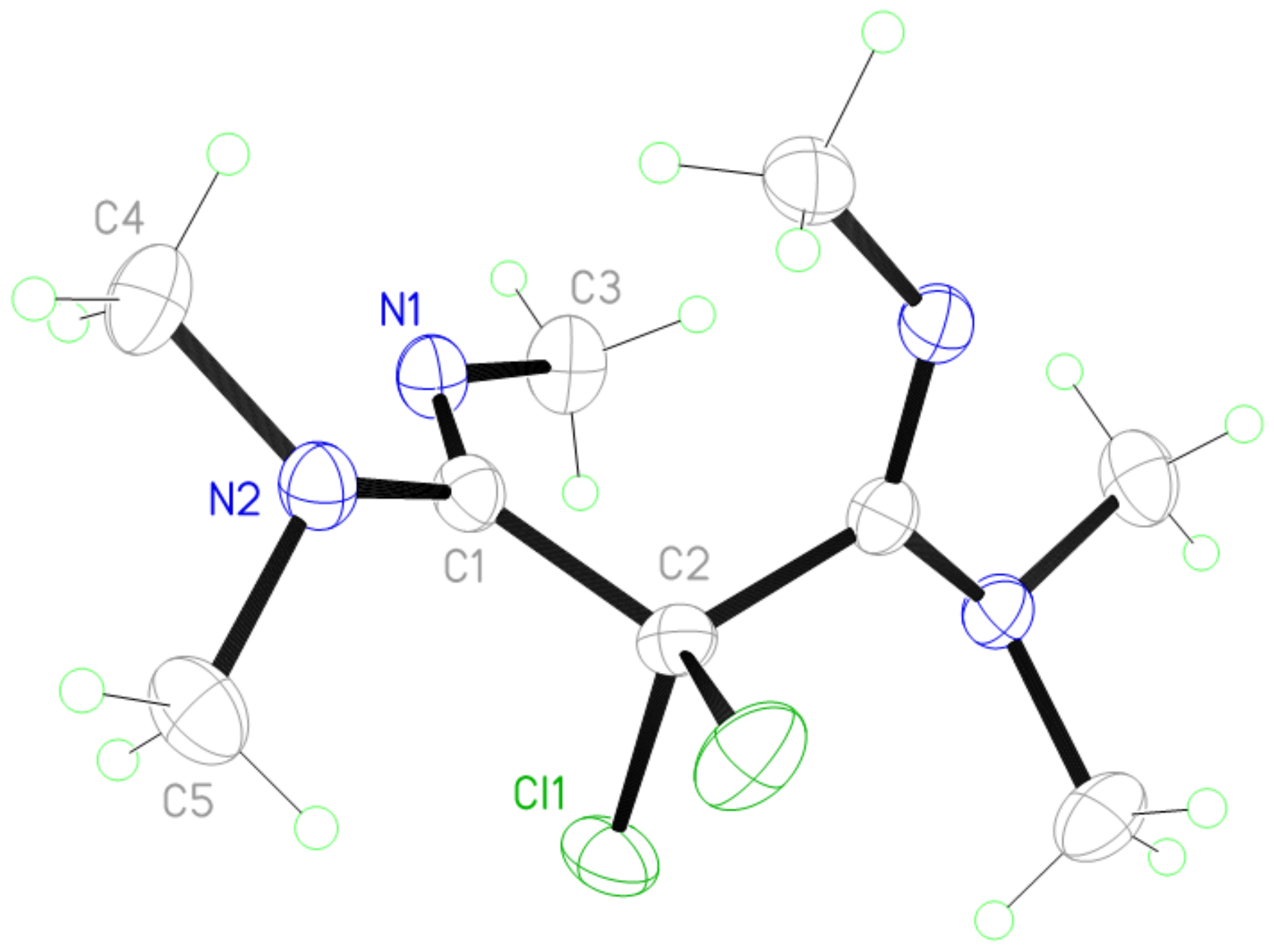

Figure S6. Molecular structure of compound 18a according to X-ray diffraction study. Thermal ellipsoids are shown with $50 \%$ probability level. 
Crystal data for compound 18a: $\mathrm{C}_{9} \mathrm{H}_{38} \mathrm{Cl}_{2} \mathrm{~N}_{4}, \mathrm{M}=253.17$, orthorhombic, space group $C 222_{1}, a=12.879(2), b=24.758(4)$, $c=7.8446(10) \AA, \mathrm{V}=2501.3(7) \AA^{3}, \mathrm{Z}=8, \mathrm{~d}_{\mathrm{c}}=1.345 \mathrm{~g} \cdot \mathrm{cm}^{-3}, \mu=0.495 \mathrm{~mm}^{-1}, \mathrm{~F}(000)=1072$, crystal size ca. $0.33 \times 0.46$ $\times 0.48 \mathrm{~mm}$. All crystallographic measurements were performed at ambient temperature on a Bruker Smart Apex II diffractometer operating in the $\omega$ scans mode. The intensity data were collected within the range of $1.65 \leq \theta \leq 26.81^{\circ}$ using Mo-K $\mathrm{K}_{\alpha}$ radiation $\left(\lambda=0.71078 \AA\right.$ ). The intensities of 13150 reflections were collected (2633 unique reflections, $R_{\text {merg }}=$ 0.0341). The structure were solved by direct methods and refined by the full-matrix least-squares technique in the anisotropic approximation for non-hydrogen atoms using the Bruker SHELXTL program package. ${ }^{58}$ All CH hydrogen atoms were placed at calculated positions and refined as 'riding' model. Convergence was obtained at R1 $=0.0750$ and $\mathrm{WR} 2=0.2080$ for 2336 observed reflections with $\mathrm{I} \geq 2 \sigma(\mathrm{I}), \mathrm{R} 1=0.0849$ and $\mathrm{wR} 2=0.2218$, GOF $=1.064$ for 2633 independent reflections, 137 parameters, Flack parameter $-0.00(2)$, the largest and minimal peaks in the final difference map 1.51 and $-0.73 \mathrm{e} / \AA^{3}$. 


\section{PHKG006_C13}

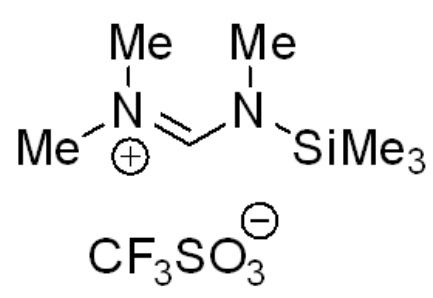

2

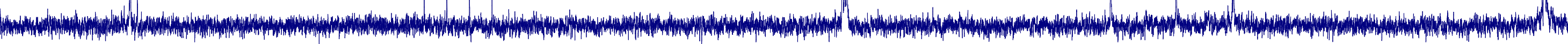

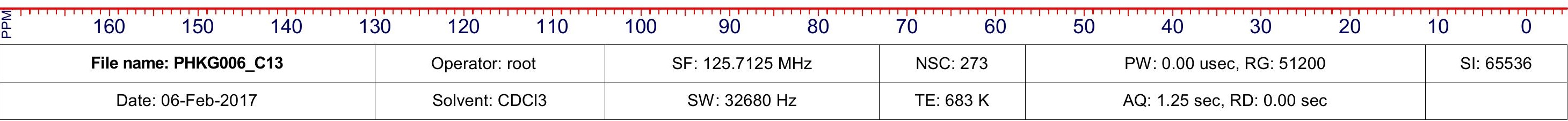




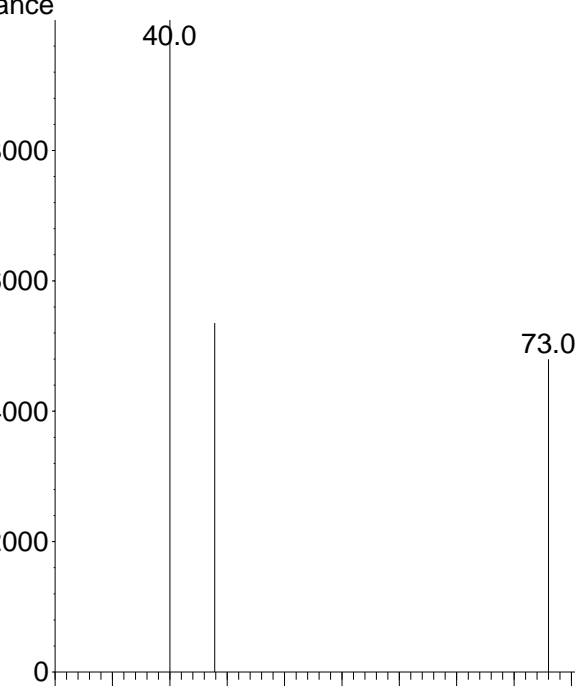

$\mathrm{m} / \mathrm{z-->}$ $30 \quad 3540 \quad 45 \quad 50 \quad 5560 \quad 6570 \quad 7580 \quad 8590 \quad 95100105110115120125130135140145150155160165170175180185190195200205210215220225$

Data File: C: \msdchem \1\data \11_05\IOH49984.D Sample : IOH49984

Peak Number: 1 at $3.557 \mathrm{~min}$ Area: 21467 Area 0.04

The 3 best hits from each library. The 3 best hits from each library. Ref $\backslash \# \quad$ CAS $\backslash \#$ Qual The 3 best hits from each library. Ref $\backslash \#$ CAS $\backslash \#$ Qual The 3 best hits from each library. Ref $\backslash \#$ CAS $\backslash \#$ Qual C: \Database $\backslash$ EMPTY.L No hits were retrieved.

S27

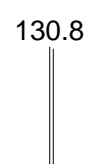

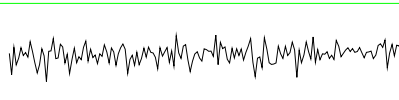

$\begin{array}{llllll}3.20 & 3.40 & 3.60 & 3.80\end{array}$ $\mathrm{m} / \mathrm{z} 219.00 \quad 50.05 \%$ 219.0
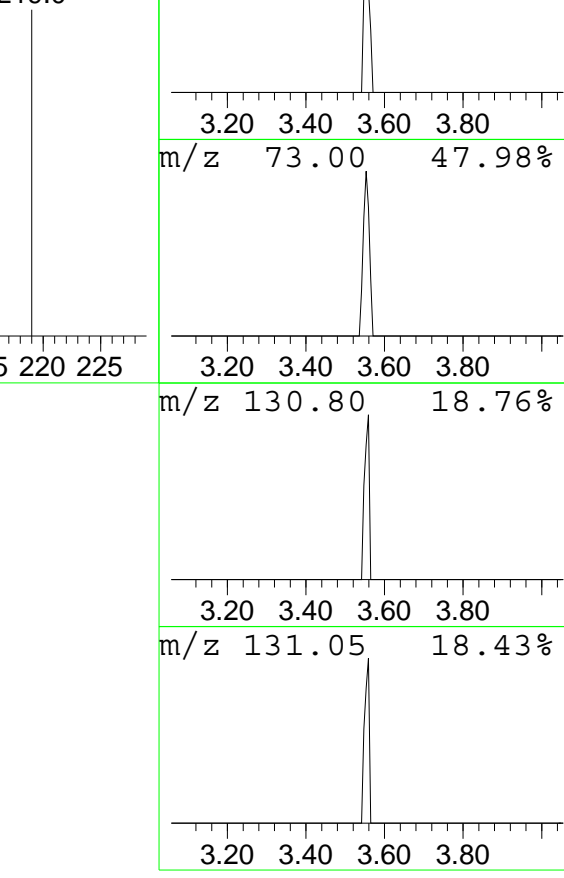

$\begin{array}{llll}3.20 & 3.40 & 3.60 & 3.80\end{array}$

$\mathrm{m} / \mathrm{z} \quad 73.00 \quad 47.98 \%$

$\begin{array}{llll}3.20 & 3.40 & 3.60 & 3.80\end{array}$ 


\section{phkg005.fid}

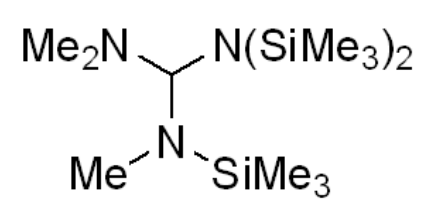

3

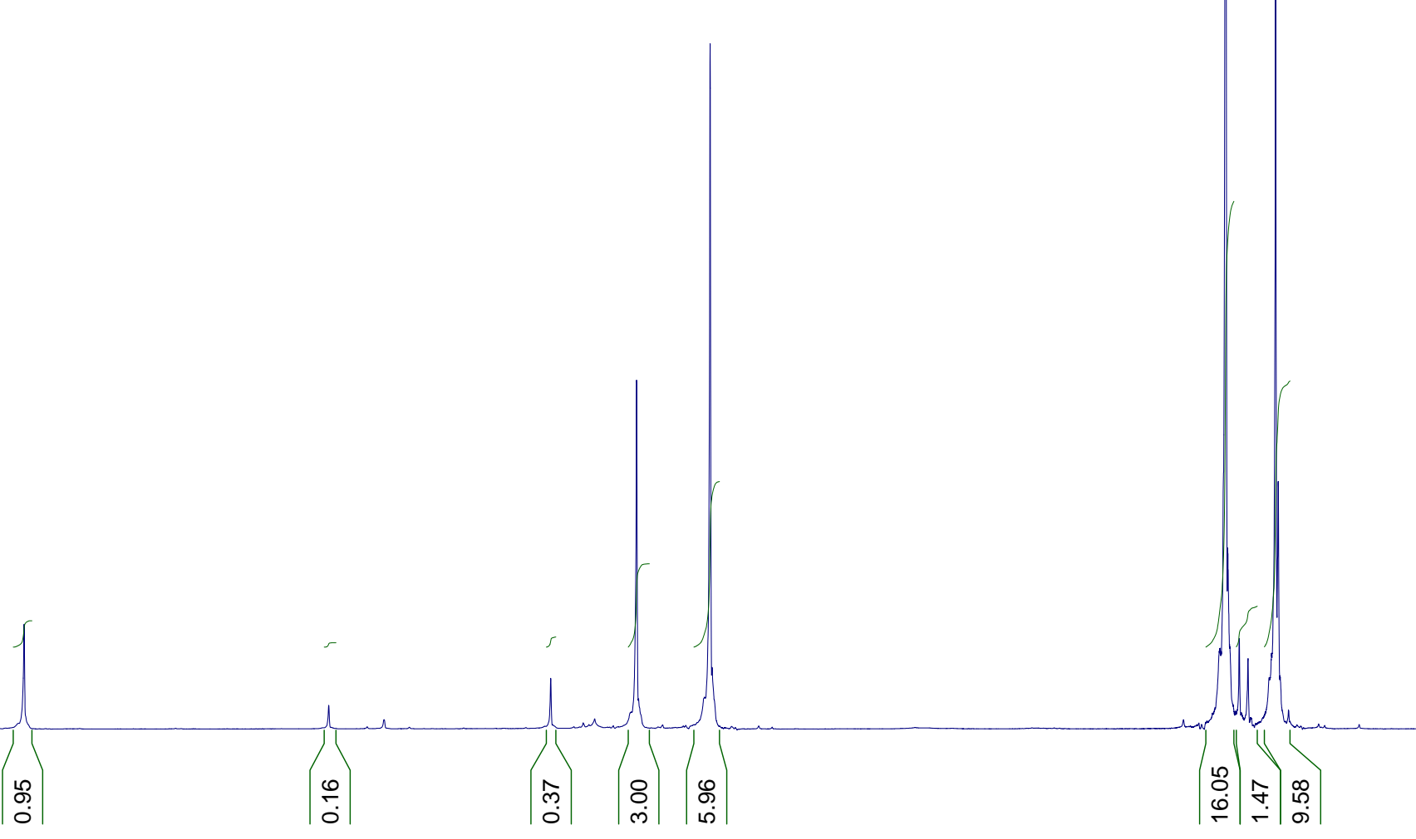

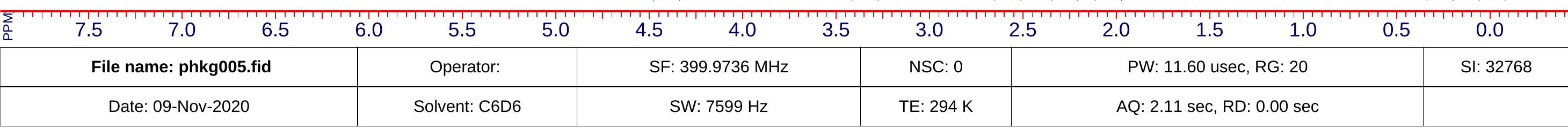




\section{PHKG005_C13}

$\mathrm{Me}_{2} \mathrm{~N} Y \mathrm{~N}\left(\mathrm{SiMe}_{3}\right)_{2}$

$\mathrm{Me}^{-\mathrm{N}}-\mathrm{SiMe}_{3}$

3

$\sum_{0}^{5}$

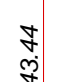

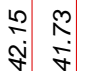

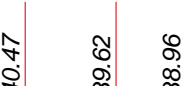

ने ले ले

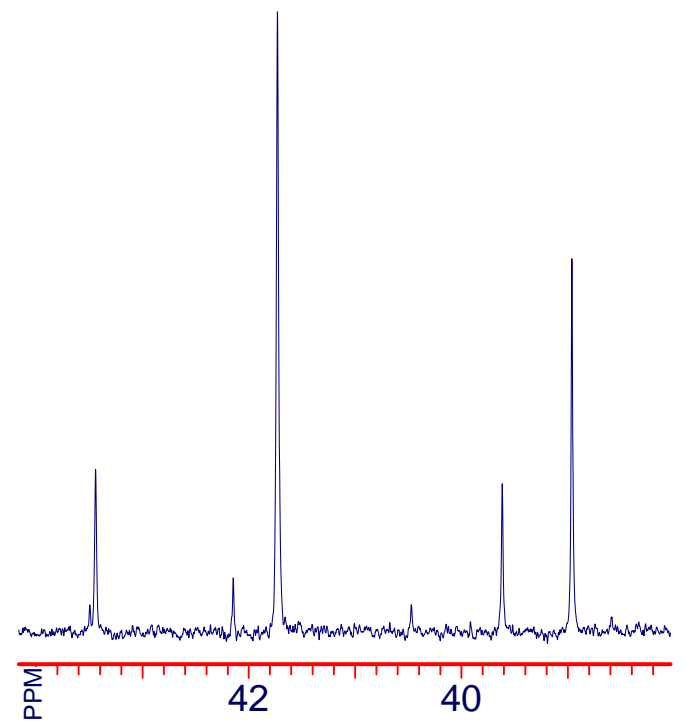

$\sum_{0}^{\infty}$

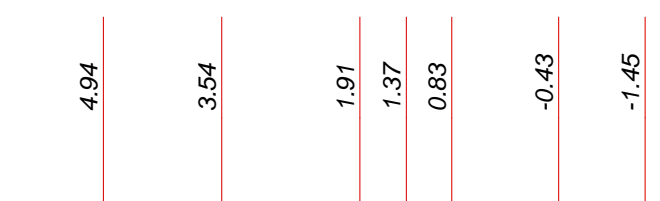

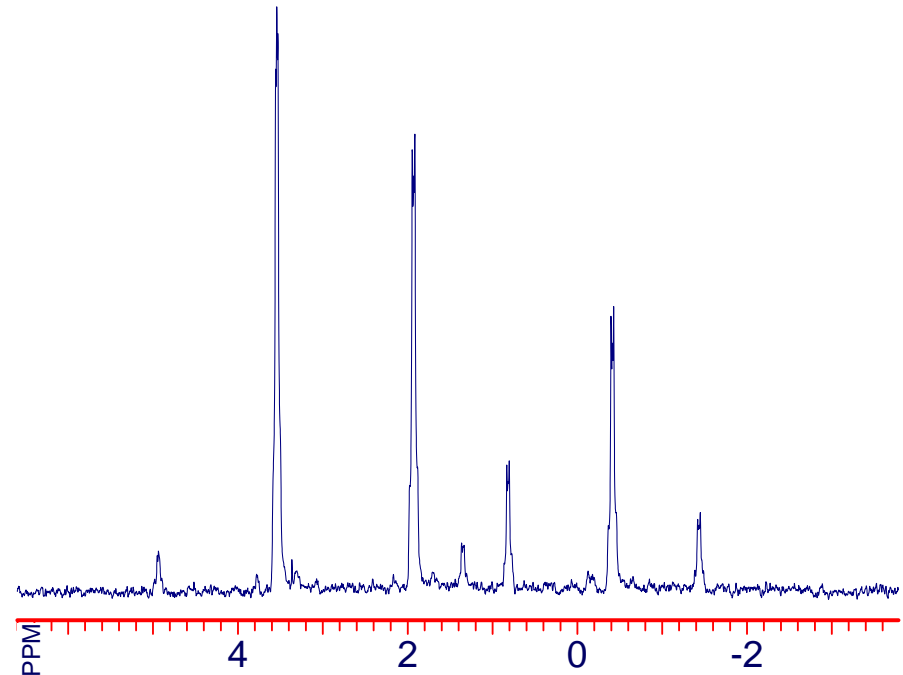

n

$\begin{array}{lllll}170 & 160 & 150 & 140\end{array}$

120

$110 \quad 100$

$90 \quad 80$

70

60

50

4030

$30 \quad 20$

$20 \quad 10$

0

File name: PHKG005_C13

Operator: root

SF: $125.6681 \mathrm{MHz}$

PW: 0.00 usec, RG: 51200

SI: 131072 


\title{
PHKG005_C13APT
}

\author{
$\mathrm{Me}_{2} \mathrm{~N}{ }_{Y} \mathrm{~N}\left(\mathrm{SiMe}_{3}\right)_{2}$ \\ $\mathrm{Me}^{-\mathrm{N}} \cdot \mathrm{SiMe}_{3}$
}

3

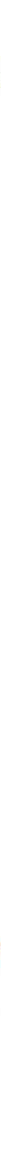




\section{KG003}

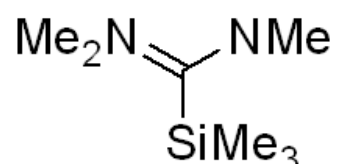

4

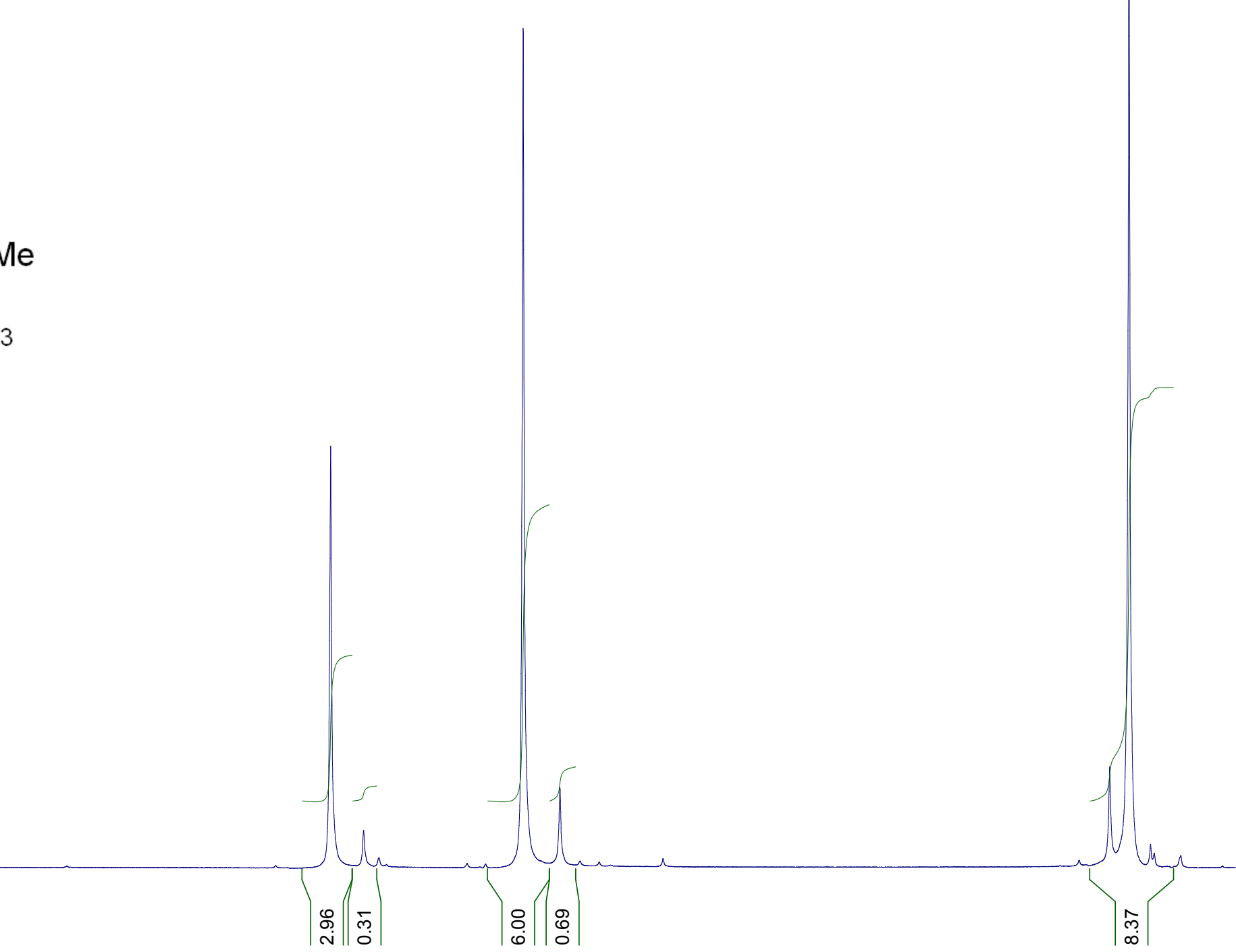

$\begin{array}{lllllllllll}7.0 & 6.5 & 6.0 & 5.5 & 5.0 & 4.5 & 4.0 & 3.5 & 3.0 & 2.5\end{array}$

PW: 4.00 usec, RG: 0

$1.0 \quad 0.5$

0.0

File name: KG003

Operator:

SF: $299.9430 \mathrm{MHz}$

NSC: 2

Q: $1.71 \mathrm{sec}, \mathrm{RD}: 2.00 \mathrm{sec}$ 


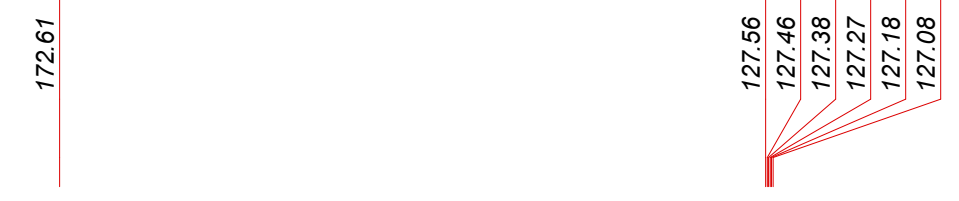

\section{PHkg003 C13}

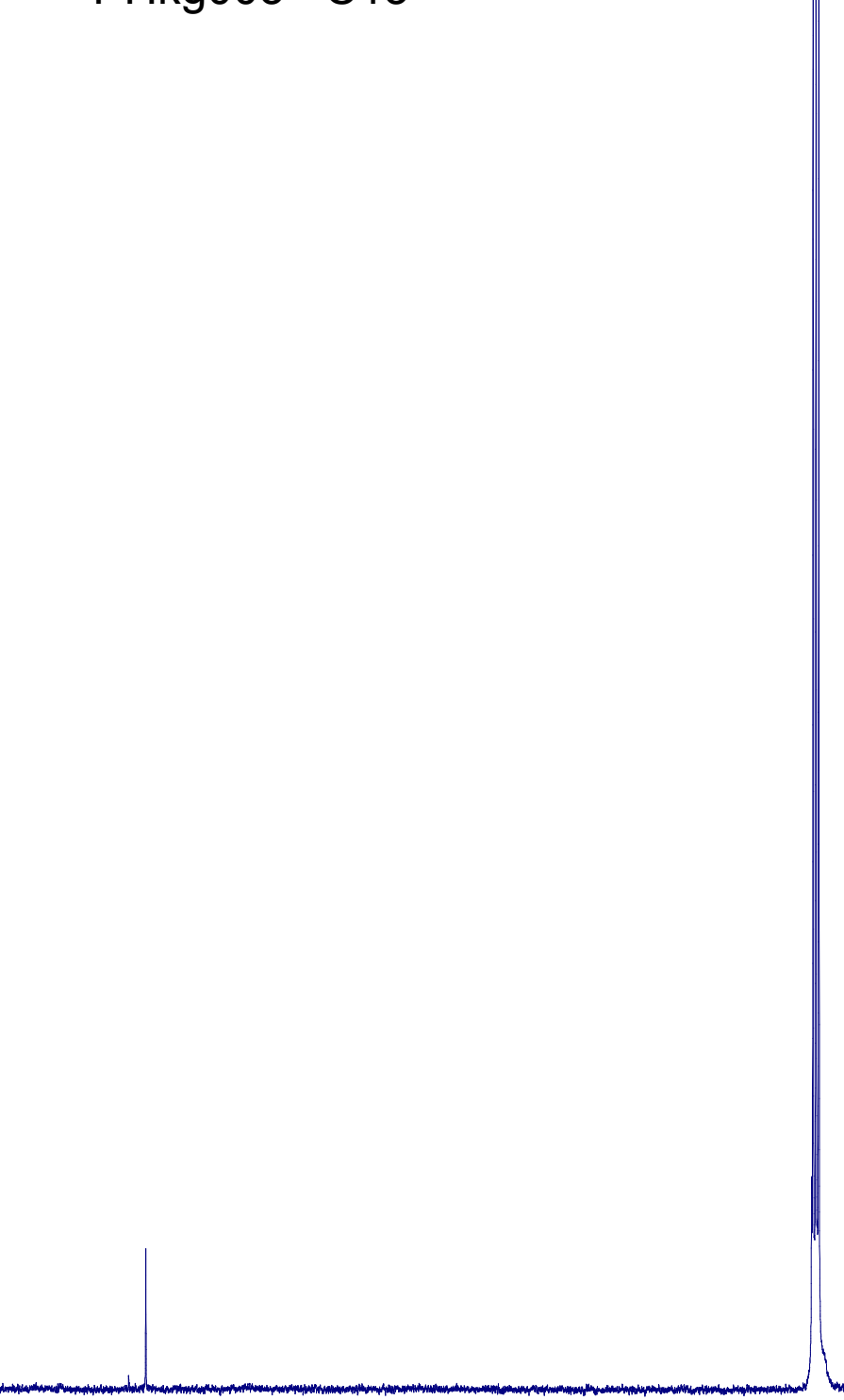

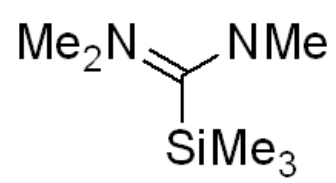

4

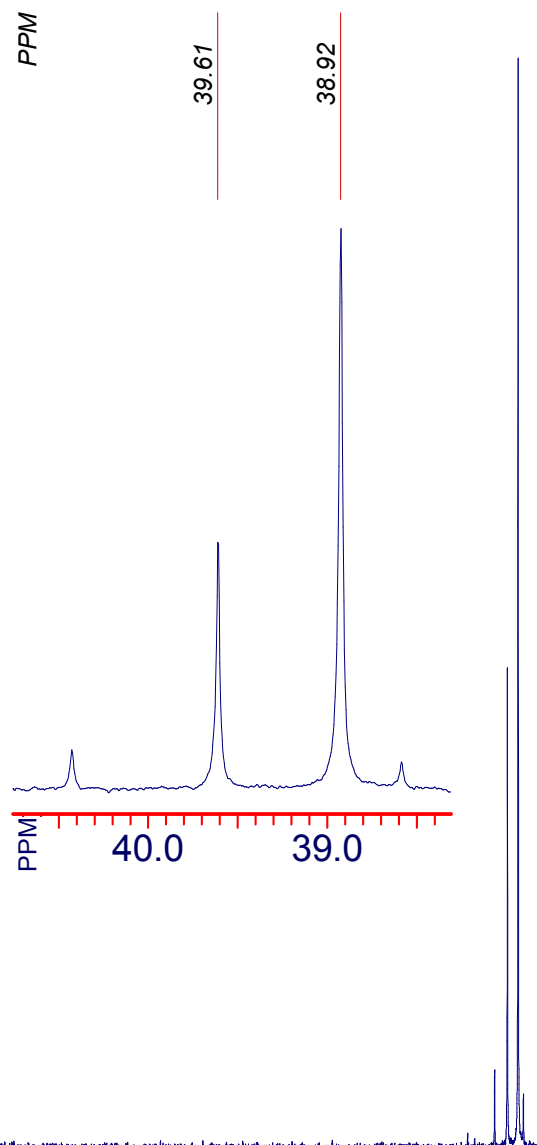

170

150

140

130

120

110

100

90

80

70

60

40

30

20

SI: 65536 
phkg043.fid

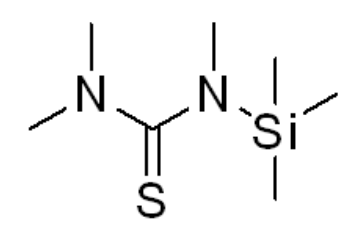

$5 a$

$\left|\begin{array}{l|l}0 \\ \stackrel{N}{0} \\ \dot{0}\end{array}\right|$

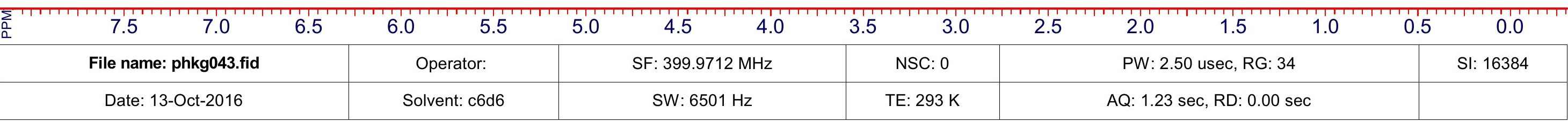




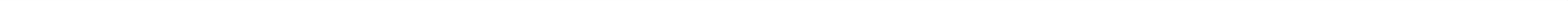




\section{PHKG010_C13}

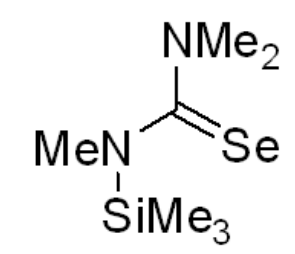

$5 b$

$\begin{array}{llllllll}\sum_{0} & 200 & 190 & 180 & 170 & 160 & 150 & 140\end{array}$

File name: PHKG010_C13

Operator: root

130

120

$110 \quad 100$

$00 \quad 90$

$90 \quad 80$

80

60

Solvent: C6D6

SF: $125.7125 \mathrm{MHz}$

NSC: 46

TE: $683 \mathrm{~K}$

$A Q: 1.25 \mathrm{sec}, \mathrm{RD}: 0.00 \mathrm{sec}$ 



\section{phkg001.fid}<smiles>CNC(=S)N(C)C</smiles>

$6 a$ 


\section{phkg001_C13}<smiles>CNC(=S)N(C)C</smiles>

$6 a$

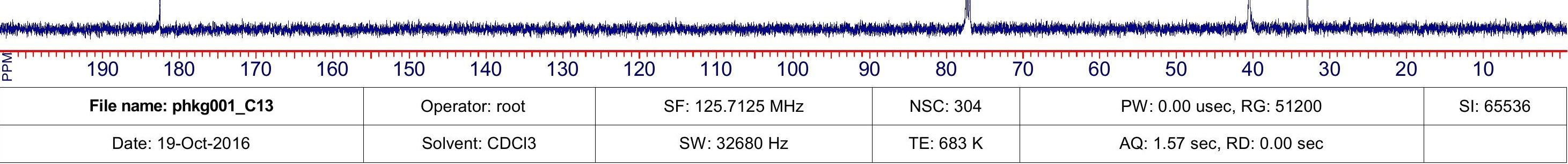




\section{phkg011.fid}

NHMe<smiles>CC(=[GeH2])N(C)C</smiles>

$6 b$

옹
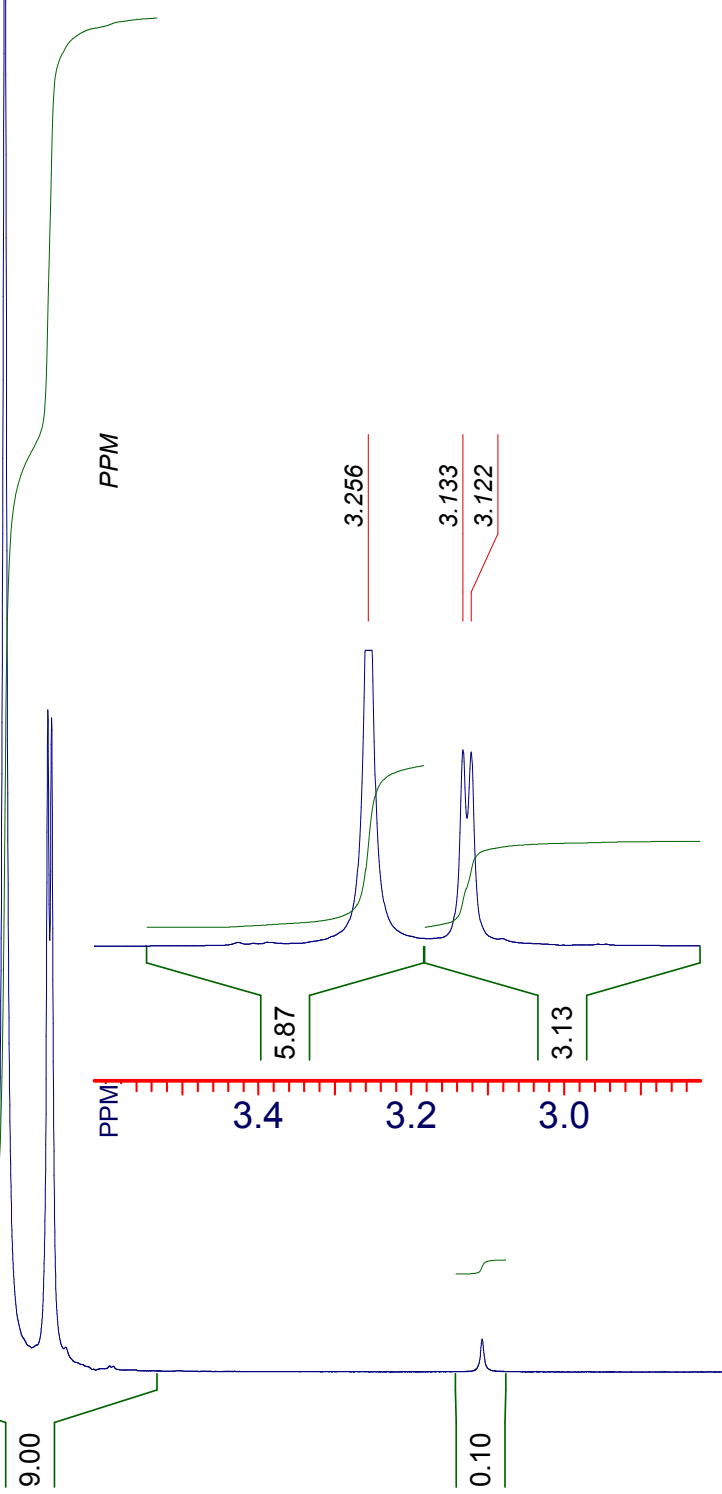

$\begin{array}{ll}7.5 & 7.0 \\ \text { File name: } & \text { phkg011.fid }\end{array}$

6.5

$6.0 \quad 5.5$

5.0

4.5

4.0

3.5

3.0

2.5

2.01 .5

1.0

PW: 7.50 usec, RG: 28

AQ: $1.20 \mathrm{sec}, \mathrm{RD}: 0.00 \mathrm{sec}$ 


\section{PHKG011_C13}<smiles>CNC(=[GeH2])NC</smiles>

6b

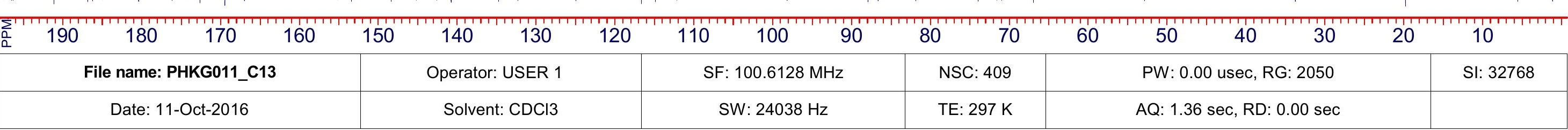


MaxPeak: $100.00 \%$

Ret_Time: $0.797 \mathrm{~min}$

\section{Mol Wt}

\section{Exact Mass}

\# Time Area\%

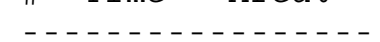

$10.797 \quad 100.00$

\section{IOH50197}

DAD1 A, Sig=215,16 Ref=off (D:IDI11_20IL308470DI005D6B-C4IOH50197.D)

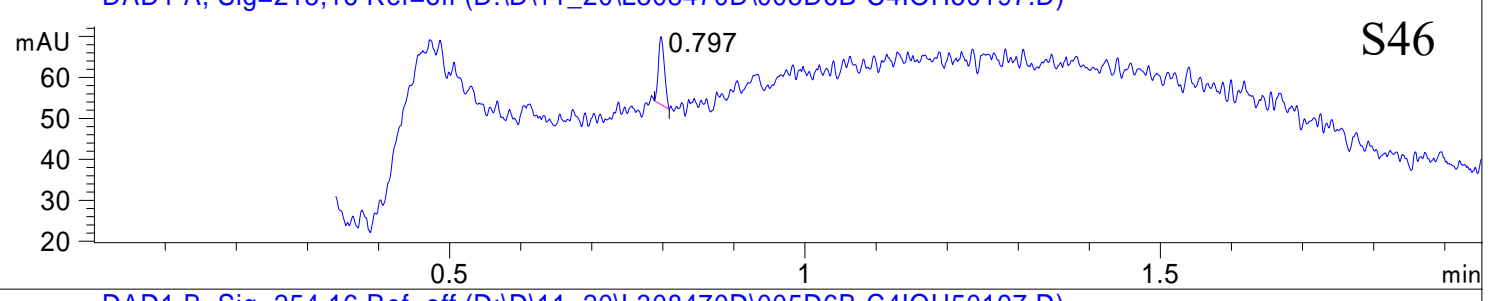

DAD1 B, Sig=254,16 Ref=off (D:IDI11_20IL308470DI005D6B-C4IOH50197.D)

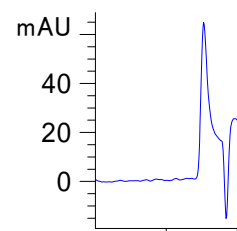

0

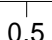

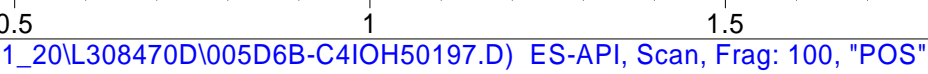
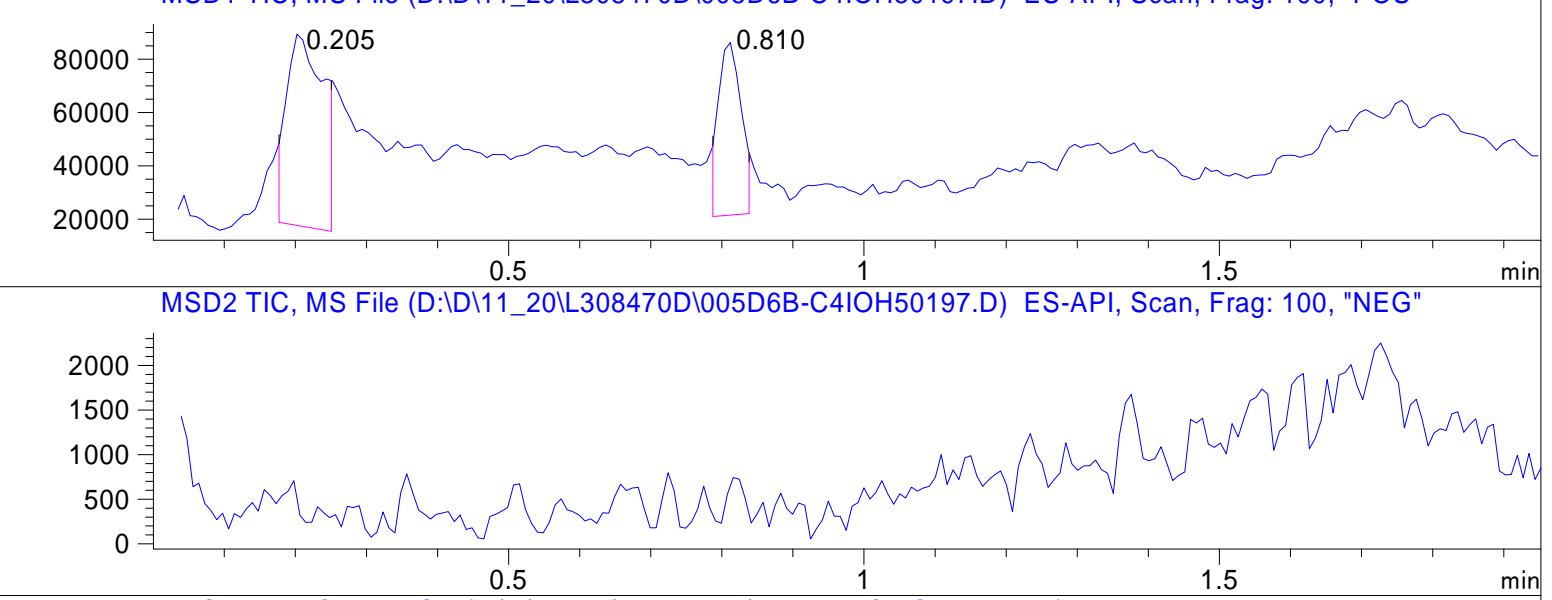

ADC1 A, ADC1A, ELSD (D:IDI11_20IL308470DI005D6B-C4IOH50197.D)

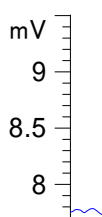

0.5

1.5

*MSD1 SPC, time=0.202 of D:IDI11_20IL308470DI005D6B-C4IOH50197.D ES-API, Scan, Frag: 100, "POS"

RT $\quad 0.205$

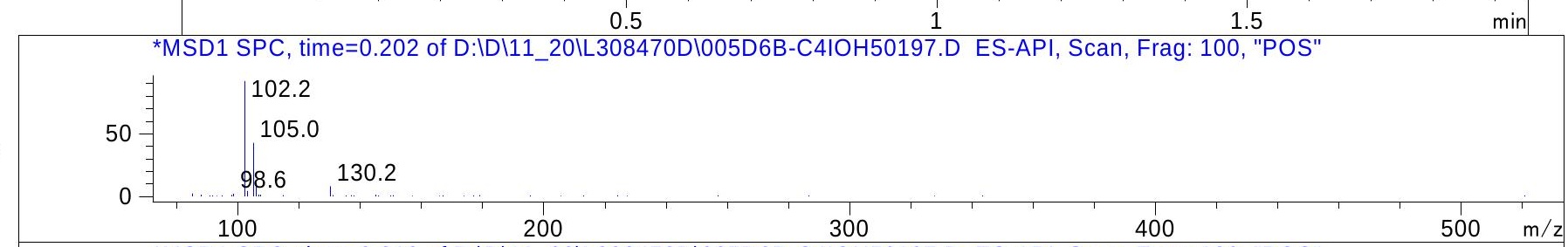

RT $\quad 0.810$

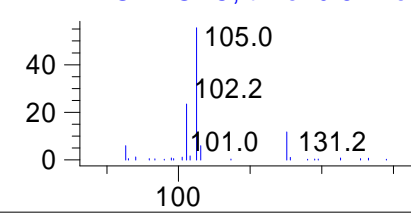

206.0

207.2

$200+300$

400

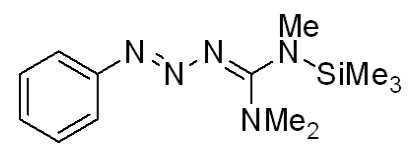


<smiles>CN/C(=N\N=Nc1ccccc1)N(C)C</smiles>

7

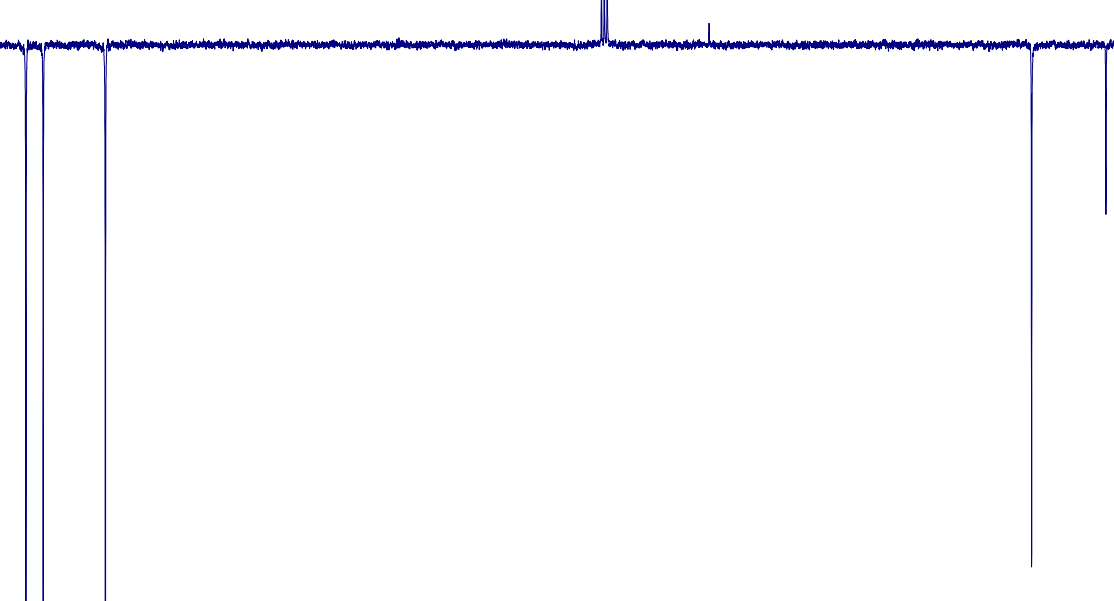

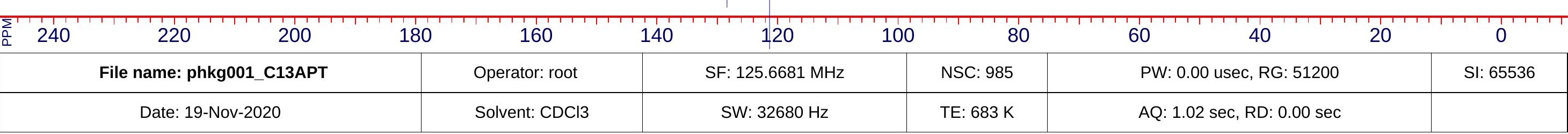


PHkg007_C13

$\mathrm{Me}^{-\mathrm{N}_{-} \mathrm{SiMe}_{3}} \mathrm{Cl}_{2} \mathrm{HC}$

8 


\section{PHkg007_APTC13}

$8 \quad \mathrm{Me}_{2}^{-\mathrm{N}} \mathrm{Cl}_{-\mathrm{SiMe}_{3}} \mathrm{NMe}_{2}$

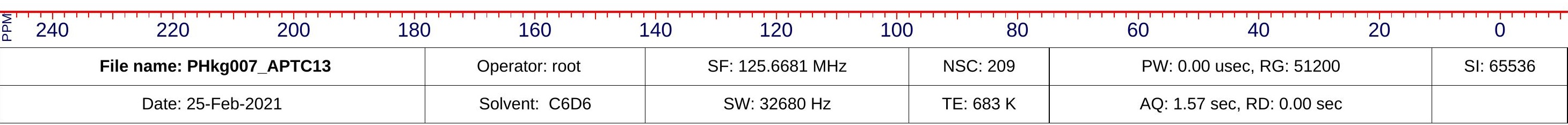




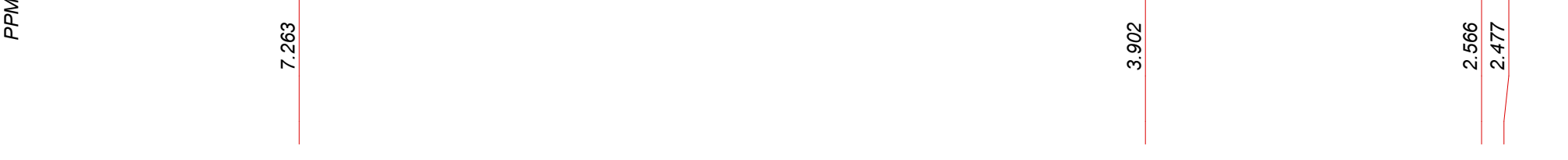

\section{S53}

\section{kg001.fid}<smiles>CN(C)C(N(C)[SiH3])C(Cl)(Cl)Cl</smiles>

9a

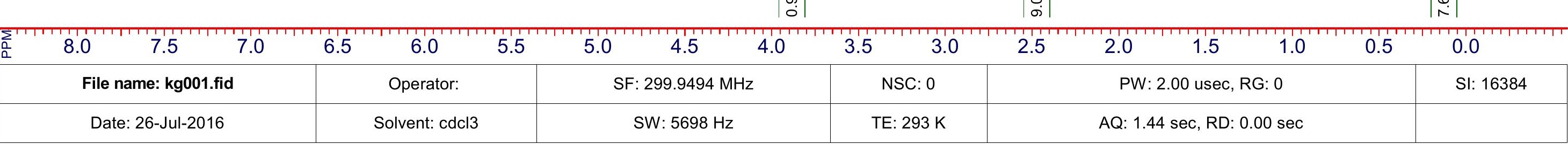




\section{PHkg011_C13}

$$
\mathrm{Cl}_{3} \mathrm{C}-\mathrm{K}_{\mathrm{N}-\mathrm{SiMe}_{3}}^{\mathrm{N}-}
$$

9a

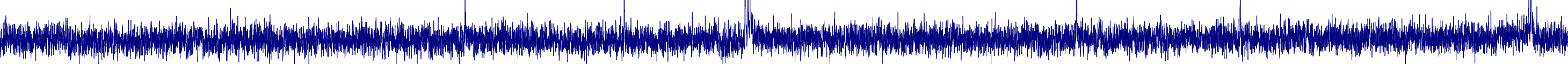

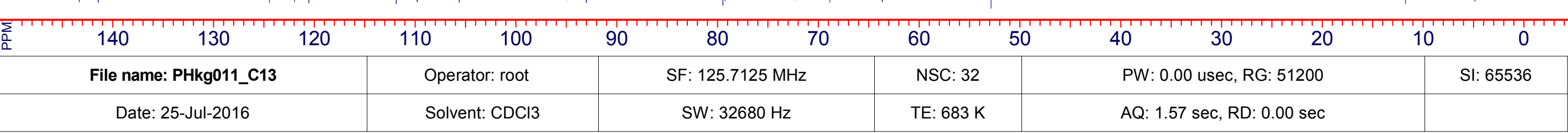




\section{PHkg011_APTC13}

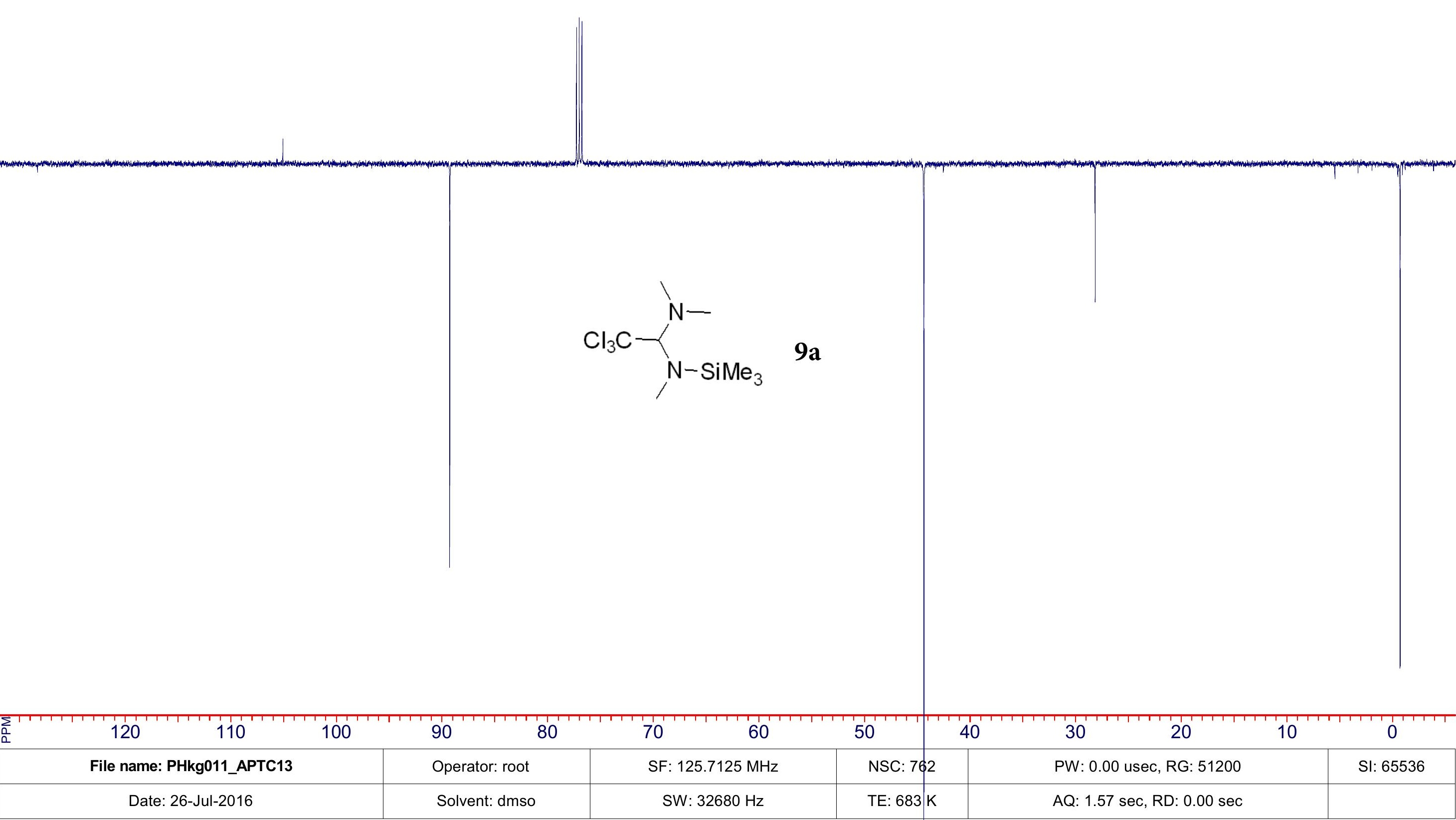




\section{PHKG003.fid}

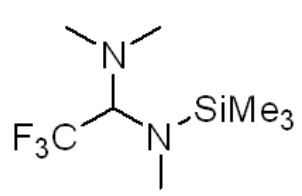

$9 b$ 


\section{PHkg003 13C}<smiles>CN(C)C(N(C)C)N(C)C(F)(F)F</smiles>

9b

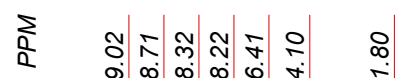

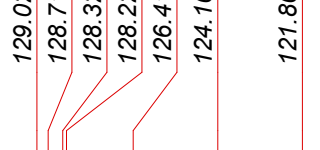

$\mid$

हn 125

120
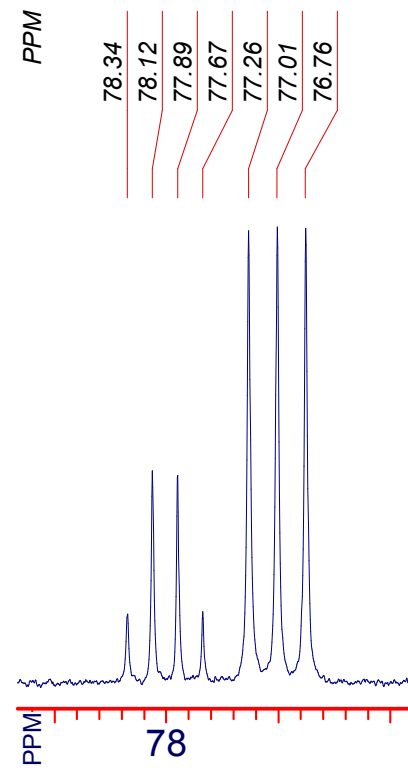

160

150

140

130

120

110

100

90

80

70

60

Operator: root

SF: $125.7125 \mathrm{MHz}$ Solvent: DMSO NSC: 418
50
40
30
20

PW: 0.00 usec, RG: 51200

$\mathrm{AQ}: 1.57 \mathrm{sec}, \mathrm{RD}: 0.00 \mathrm{sec}$ 


\section{PHkg003 APT}

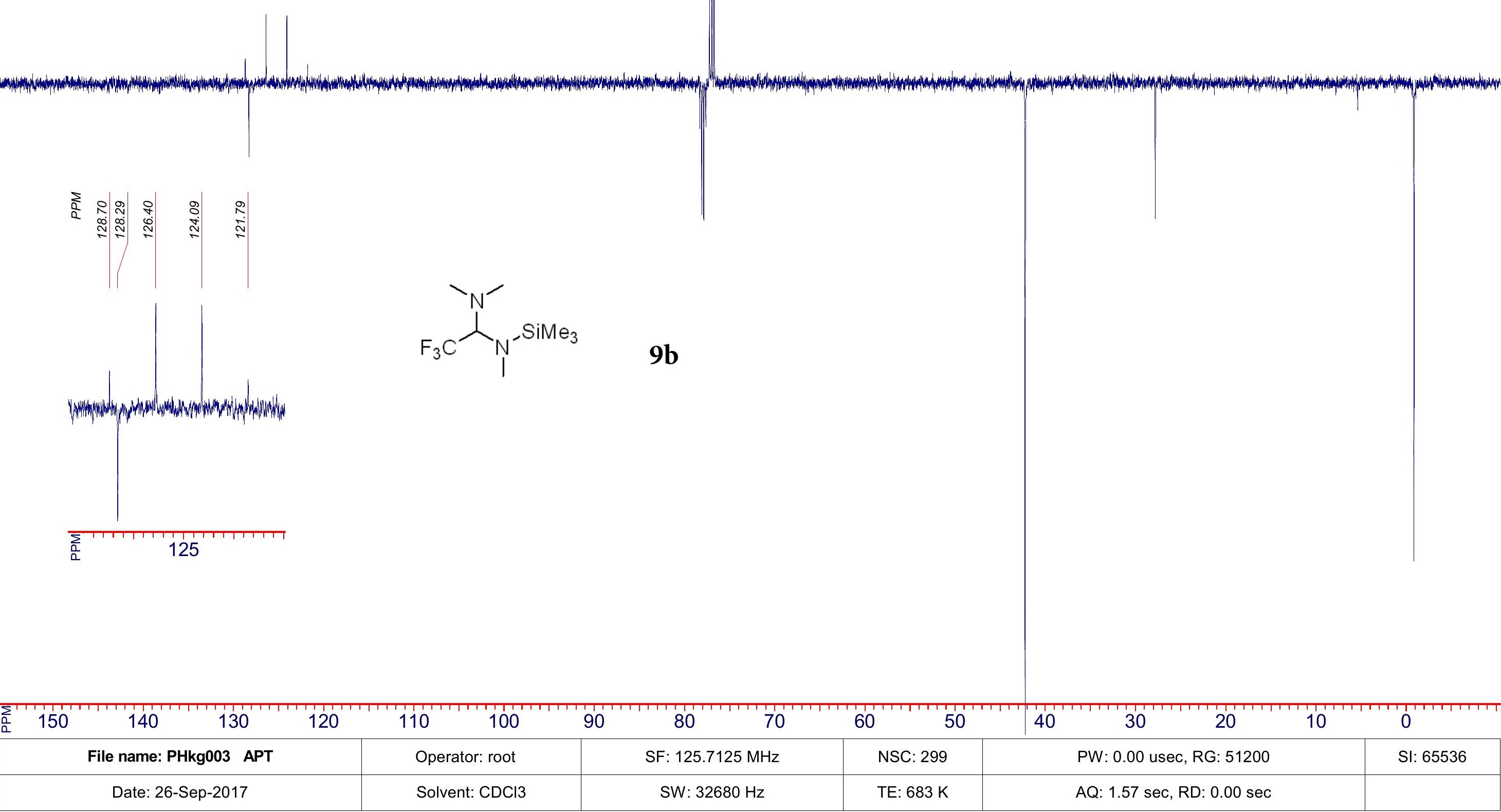


PHkg003 19F $\{1 \mathrm{H}\}$

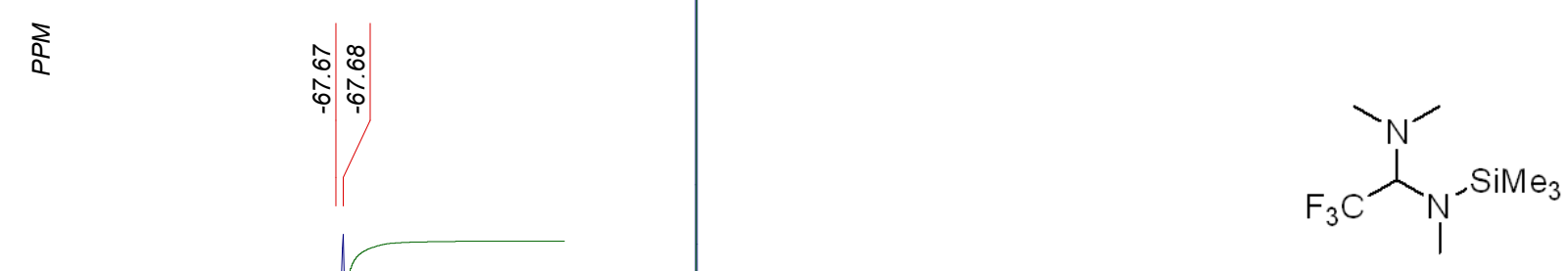

9b

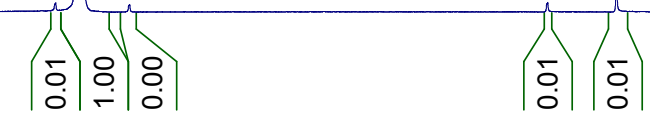

$-54$

$-56$

$-58$

$-60$

$-62$

$-64$

$-66 \quad-68$

$-70$

$-72$

$-74$

$-76$

$-78$

$-80$

SF: $376.4986 \mathrm{MHz}$

NSC: $4 \quad$ PW: 0.00 usec, RG: 2050 SW: $138889 \mathrm{~Hz}$ 


\section{PHKG003}

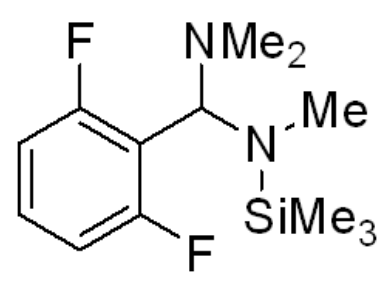

\section{$11 \mathbf{a}$}

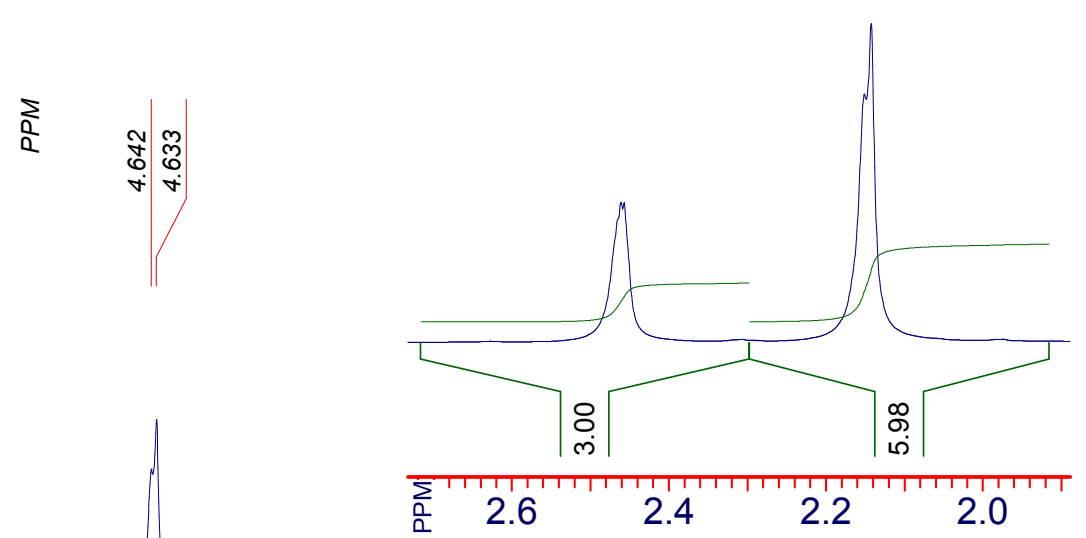

$\begin{array}{lllll}75 & 70 & 65 & 60 & 5.5\end{array}$
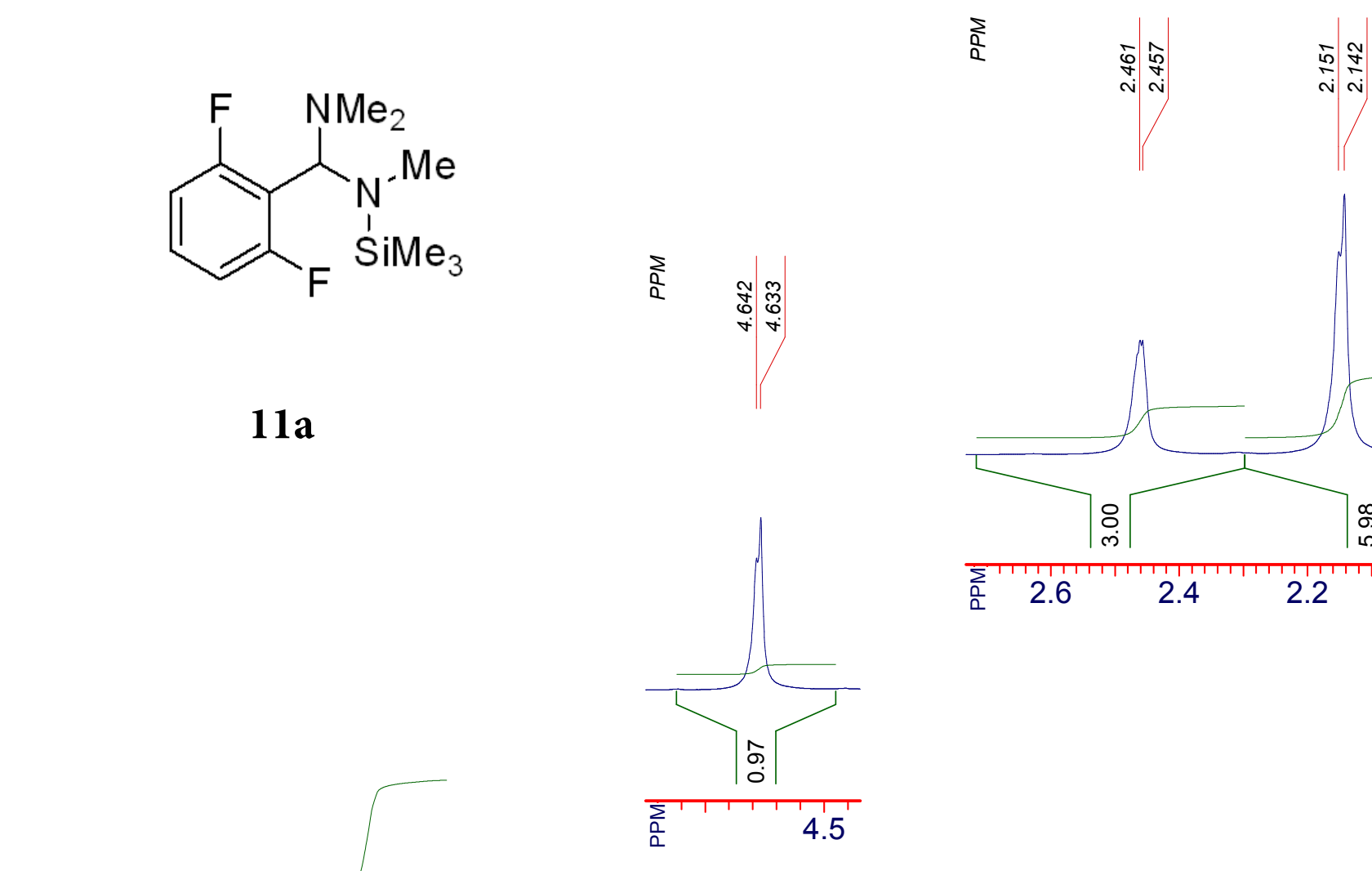

\begin{tabular}{llllll}
\hline & 4 & 4 & 3.5 & 3.0 & 2.5
\end{tabular}


phkg006.fid
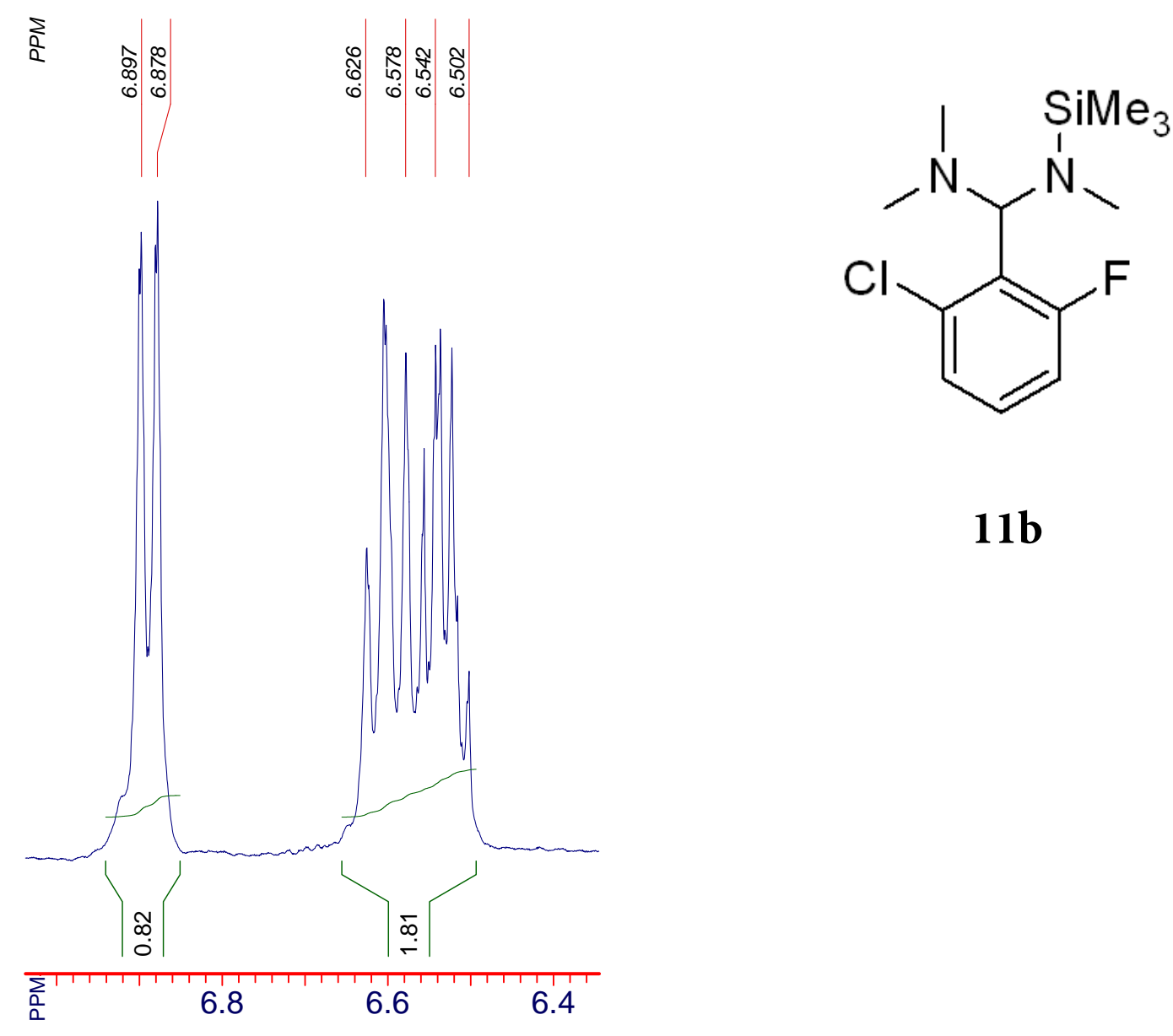

$11 b$

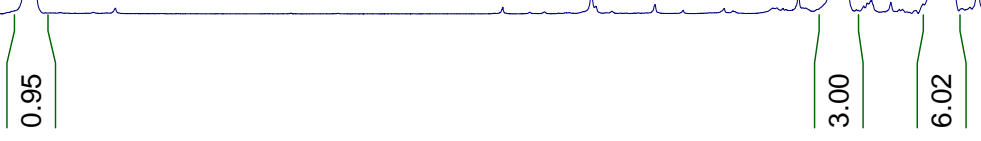

$\sum_{0}$

6.5

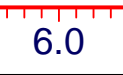

5.5

5.0

$\begin{array}{lll}4.5 & 4.0 & 3.5\end{array}$

3.0

2.5

2.0

1.5

1.0

0.5

0.0

File name: phkg006.fid

Operator:

SF: $399.9736 \mathrm{MHz}$

NSC: 0

PW: 11.60 usec, RG: 30

SI: 32768 
phkg006_C13.fid

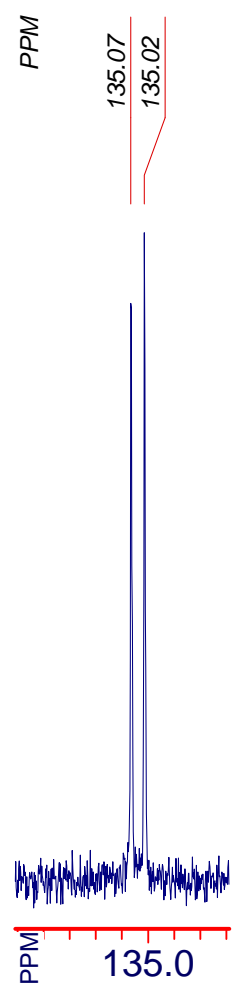

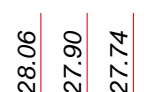

ㄱ ㄱ
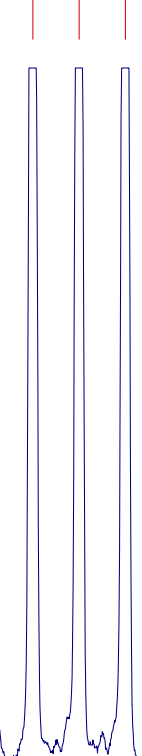

128.0
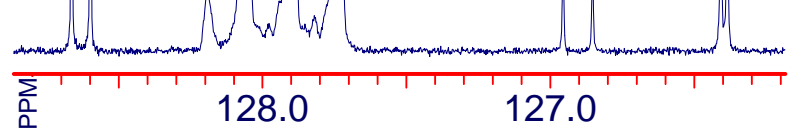

홍

일
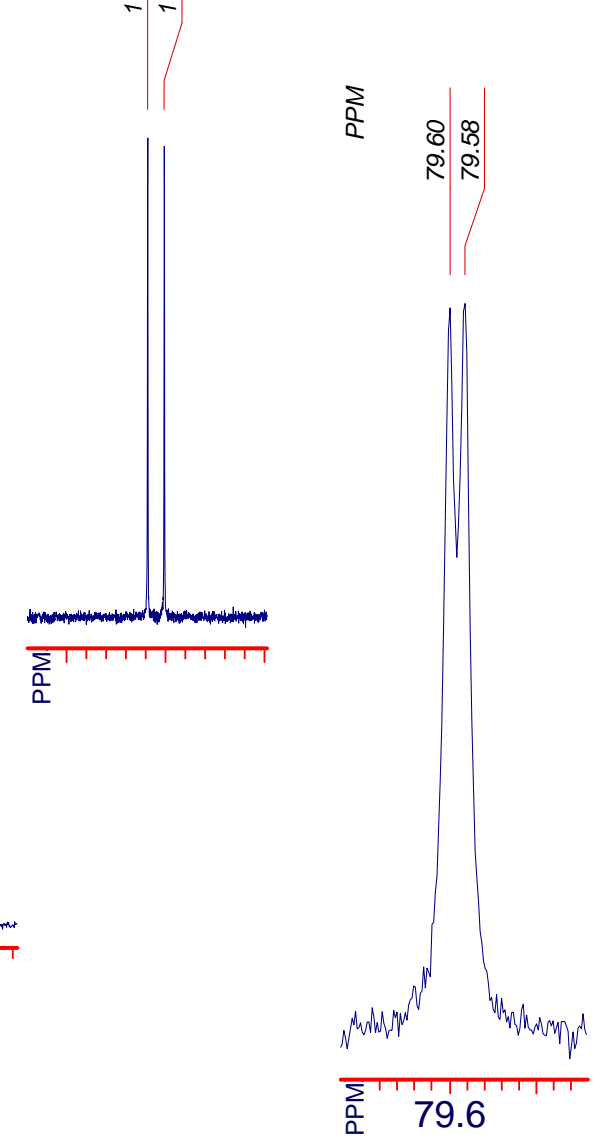<smiles>CN(C)C(c1c(F)cccc1Cl)N(C)C</smiles>

$11 b$

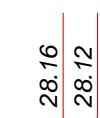

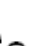

3 
phkg006_C13APT.fid<smiles>CN(C)C(c1c(F)cccc1Cl)N(C)C</smiles>

$11 b$

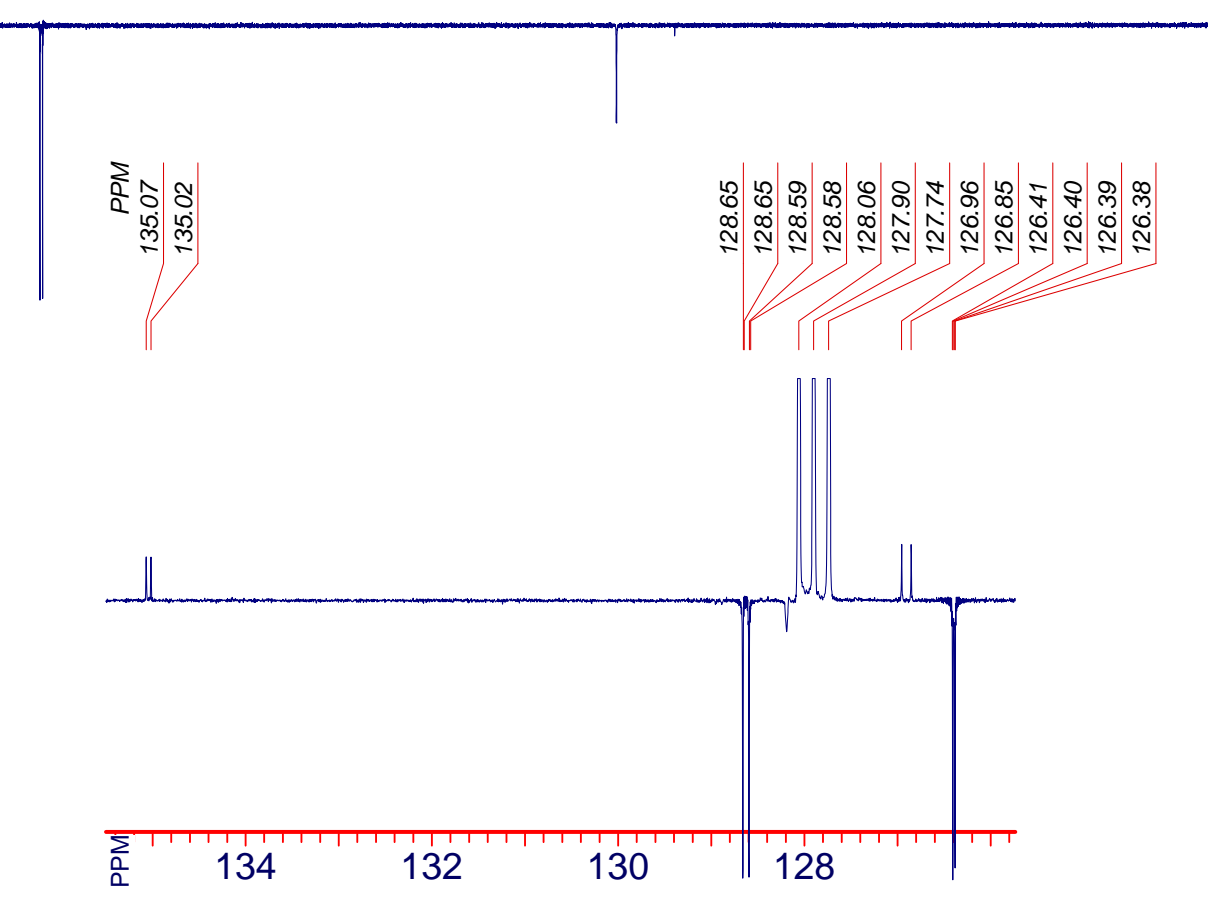

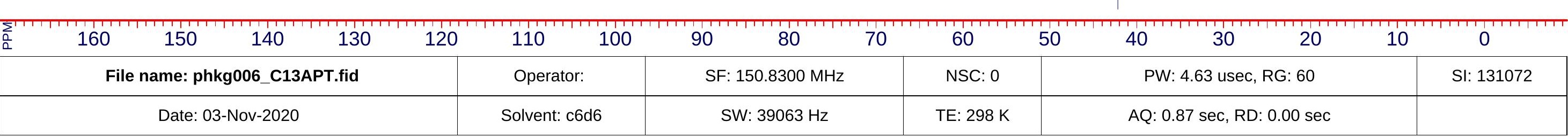




\section{phkg013.fid}
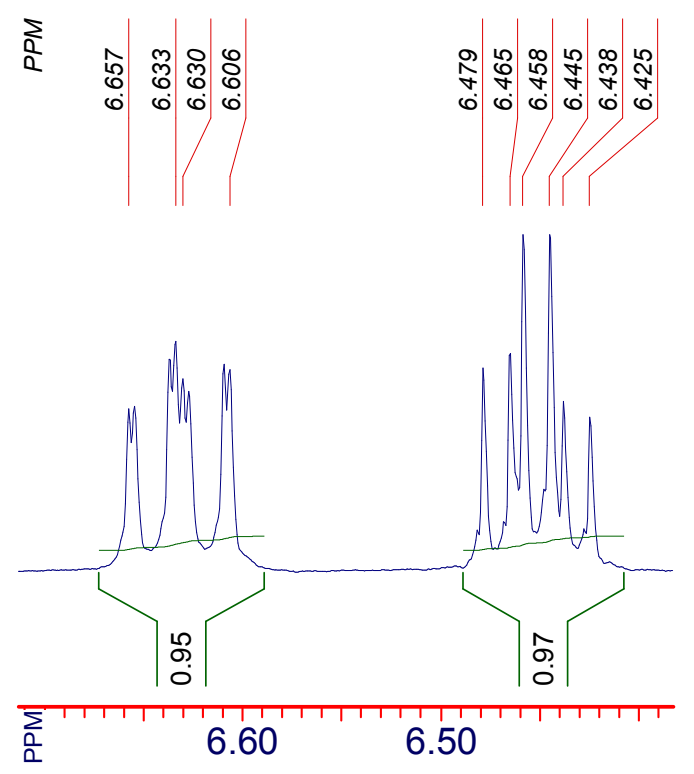

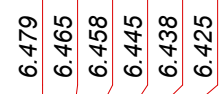

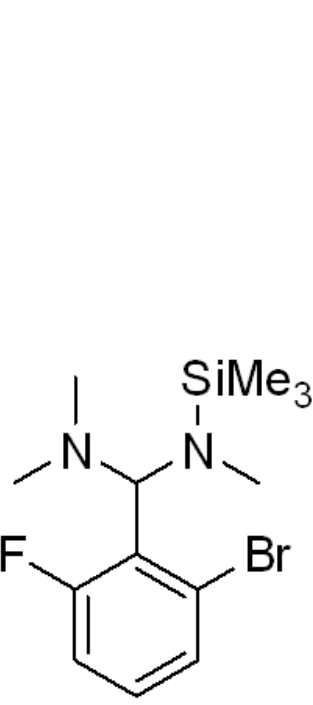

$11 b$

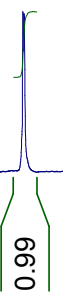

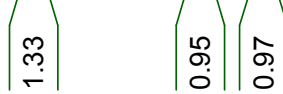

\begin{tabular}{l|l|l|}
8 & 8 \\
$\dot{m}$ & 0 \\
\hline 0
\end{tabular}

6.5

$6.0 \quad 5.5$

$5.0 \quad 4.5$

SF: $399.9736 \mathrm{MHz}$

Operator:

SW: $7599 \mathrm{~Hz}$

TE: $294 \mathrm{~K}$

PW: 11.60 usec, RG: 20

AQ: $2.11 \mathrm{sec}, \mathrm{RD}: 0.00 \mathrm{sec}$ 


\section{phkg013_C13}

$\sum_{\substack{0 \\ 0}}^{2}$
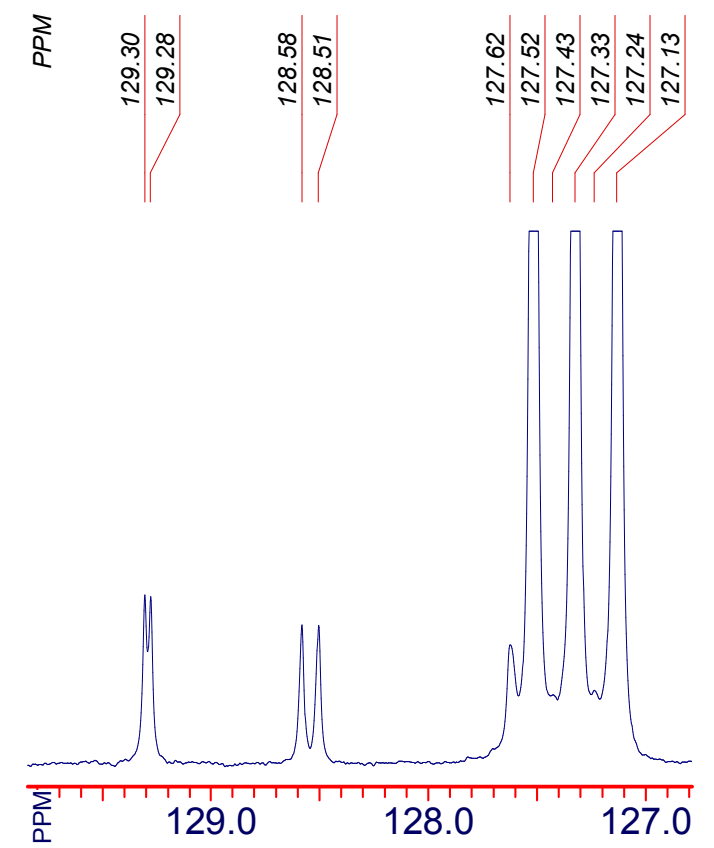

$\sum_{0}^{2}$
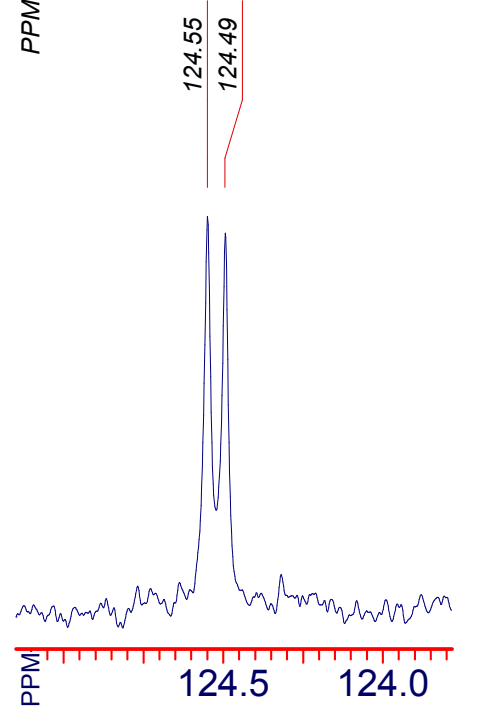<smiles>CN(C)C(c1c(F)cccc1Br)N(C)C</smiles>

11c $\sum_{0}^{\infty}$

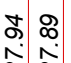

ลิ

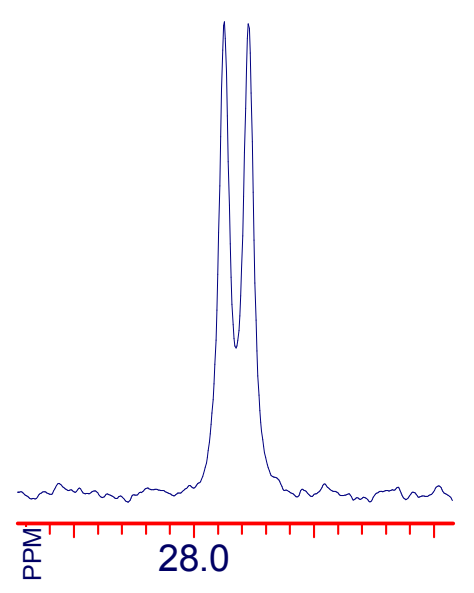

$$
=
$$

$160 \quad 150$

File name: phkg013_C13
$140 \quad 130$

30

120

90

Operator: root

SF: $125.6681 \mathrm{MHz}$

80

Solvent: C6D6

SW: $32680 \mathrm{~Hz}$

NSC: 238

60

50

30

20

10

0 



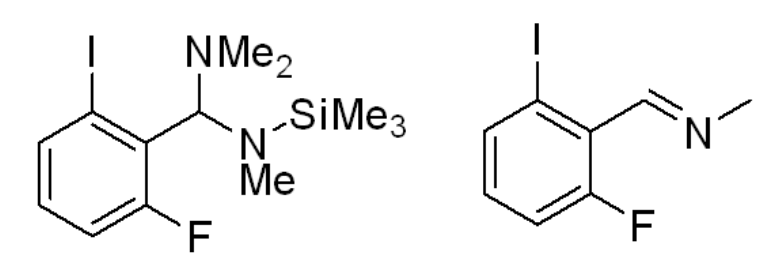<smiles>C/N=C\c1c(F)cccc1I</smiles>

\section{$11 d$}

$\frac{a}{0}$

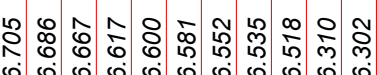
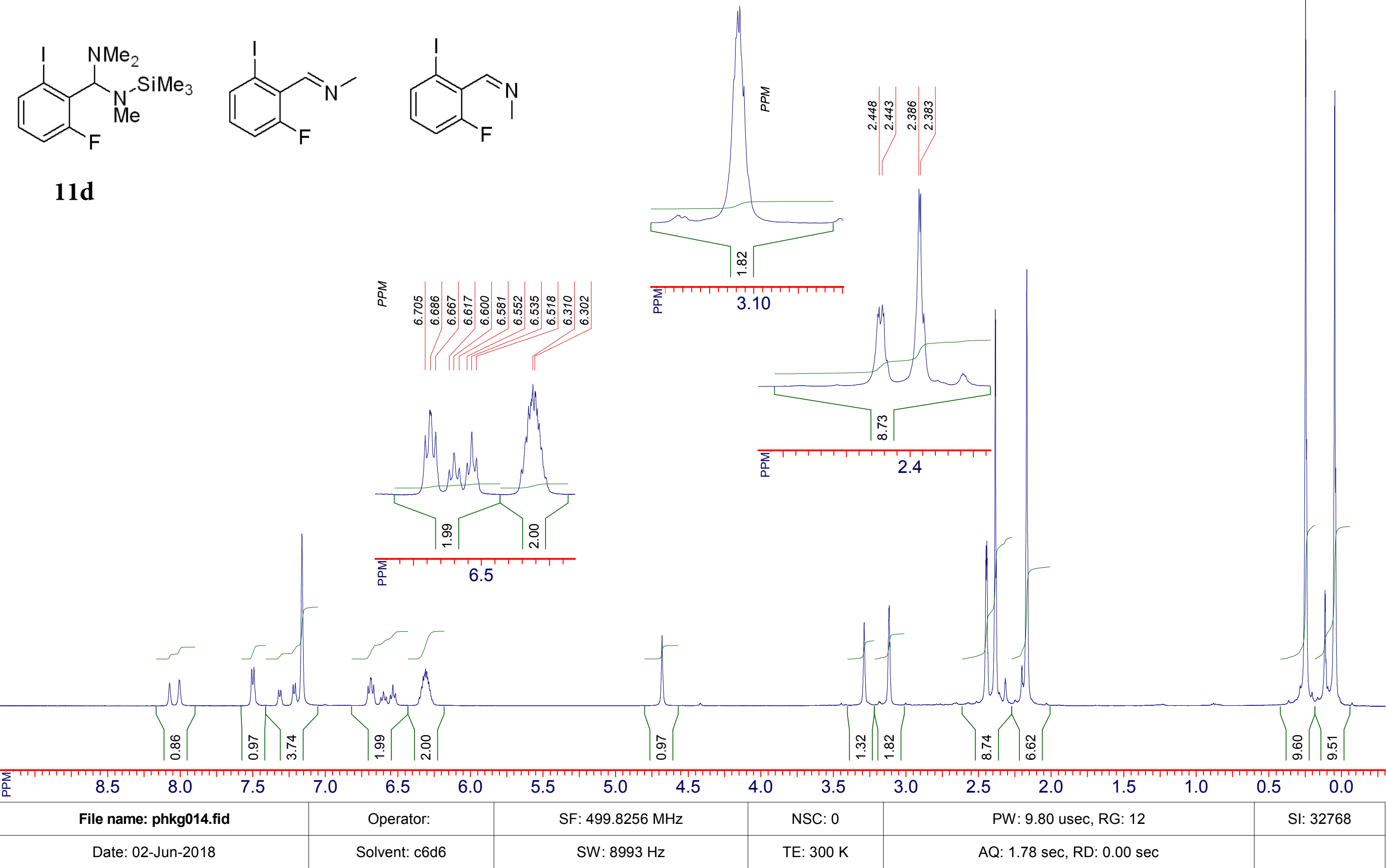

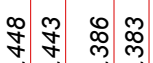

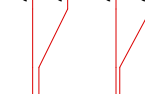




\section{phkg014_C13APT.fid}
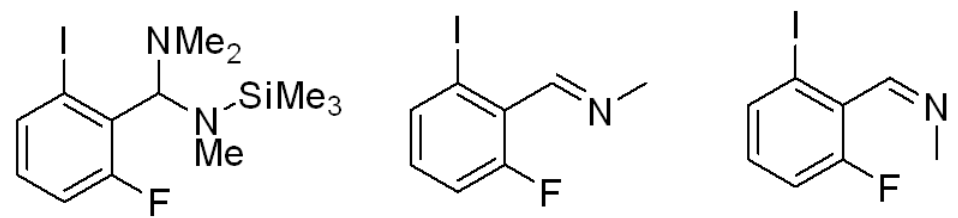

\section{1d}

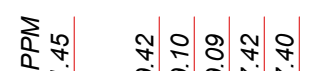

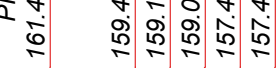

$1 /$

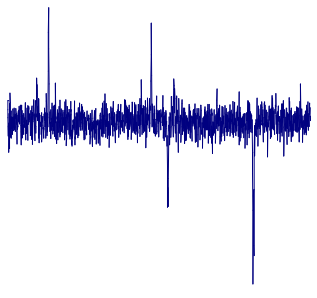

$\sum_{0} 160$

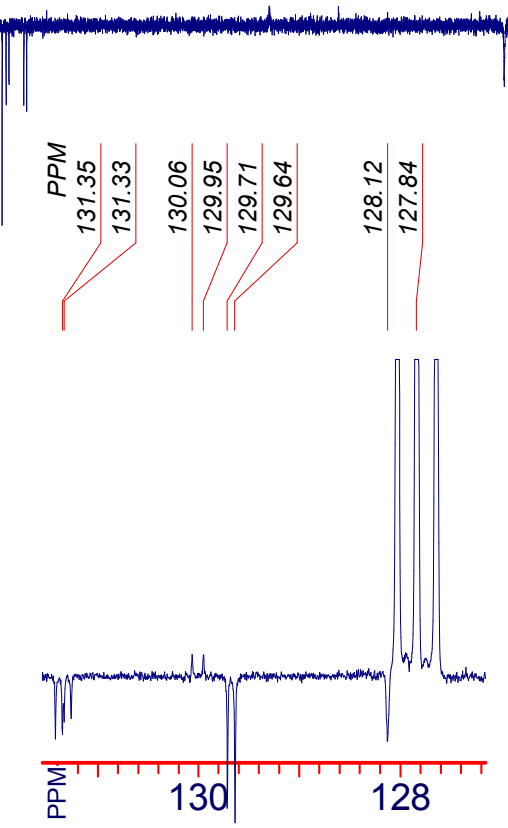

11

160

150

140

130

120

$110 \quad 100$

80

60

50

SF: $125.6954 \mathrm{MHz}$

NSC: 0

PW: 5.00 usec, RG: 60

40

30

20

10 


\section{phkg006}
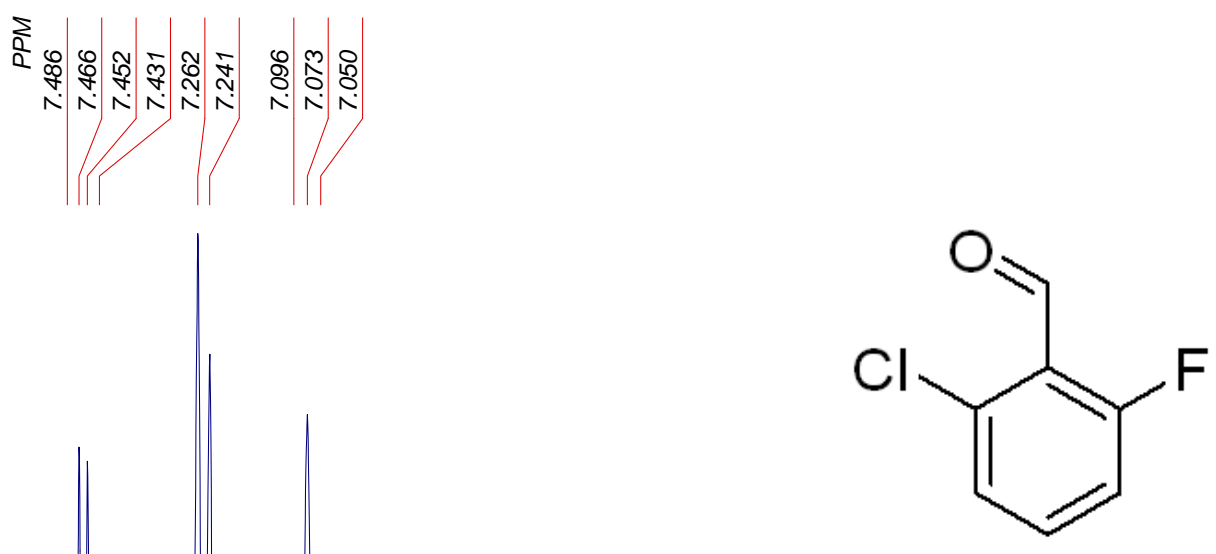

$12 b$

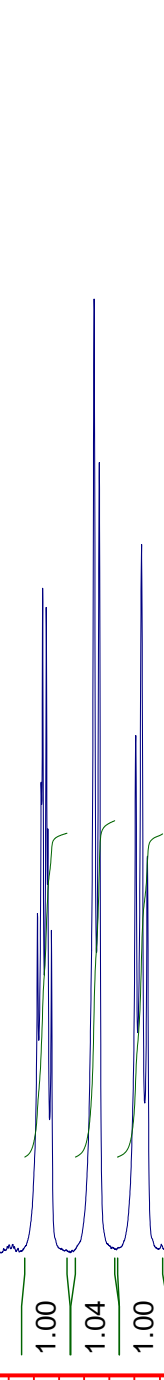

$\left|\begin{array}{l|l}8 \\ 8 \\ 4\end{array}\right| \begin{aligned} & 8 \\ & 0\end{aligned} \mid$

$\sum_{\mathrm{a}}$

7.0

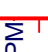

$11.0 \quad 10.0$

9.0

8.0

7.0

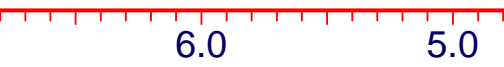

5.0

NSC: 1

TE: $297 \mathrm{~K}$

SW: $8224 \mathrm{~Hz}$

\begin{tabular}{|l} 
NSC: 1 \\
\hline$z$
\end{tabular}




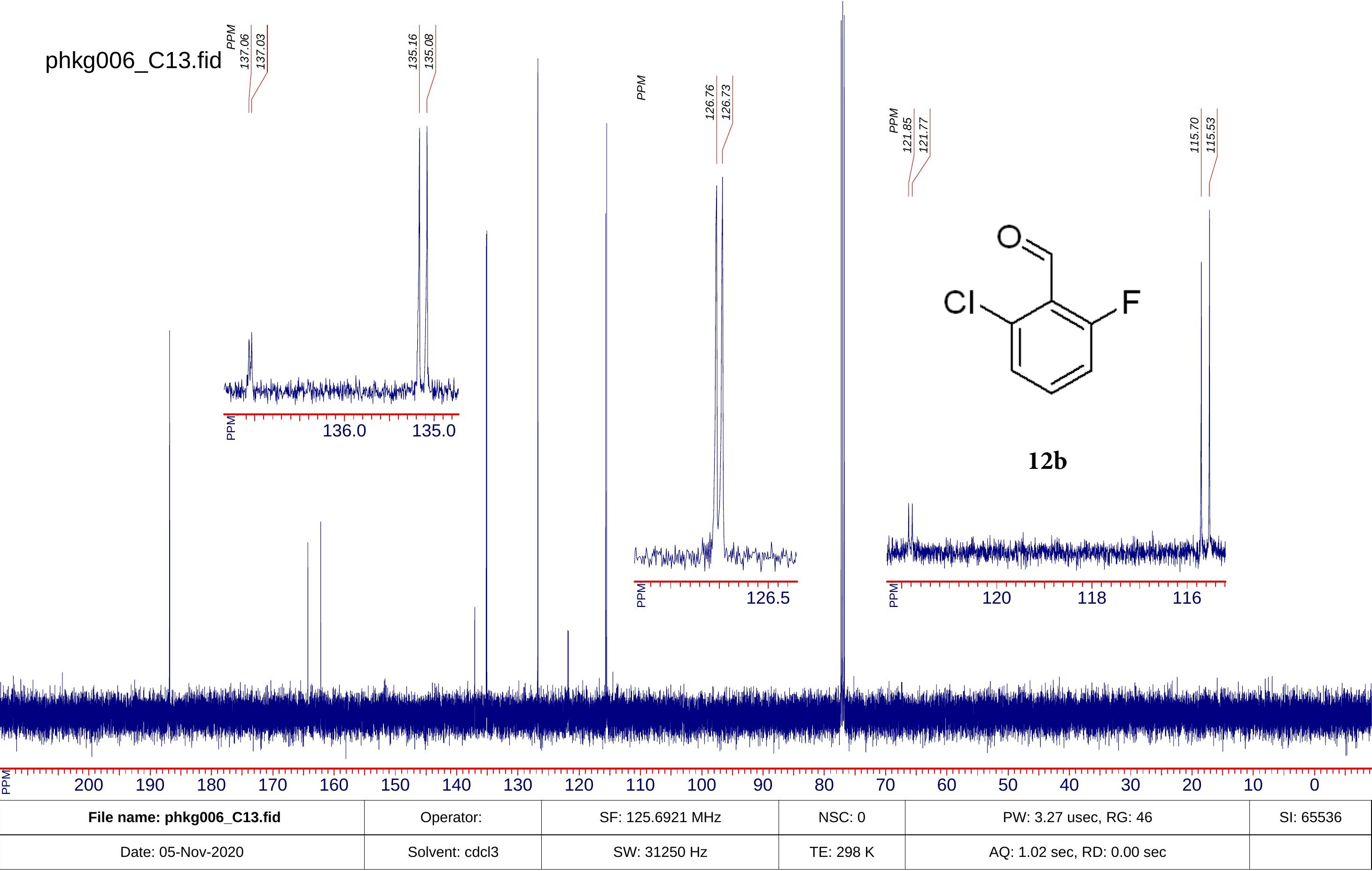




\section{phkg006_C13APT.fid}

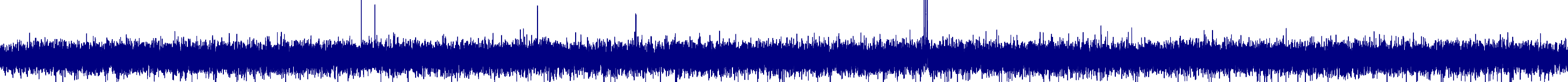

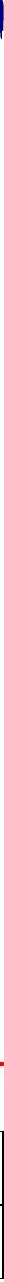




\section{phkg007}

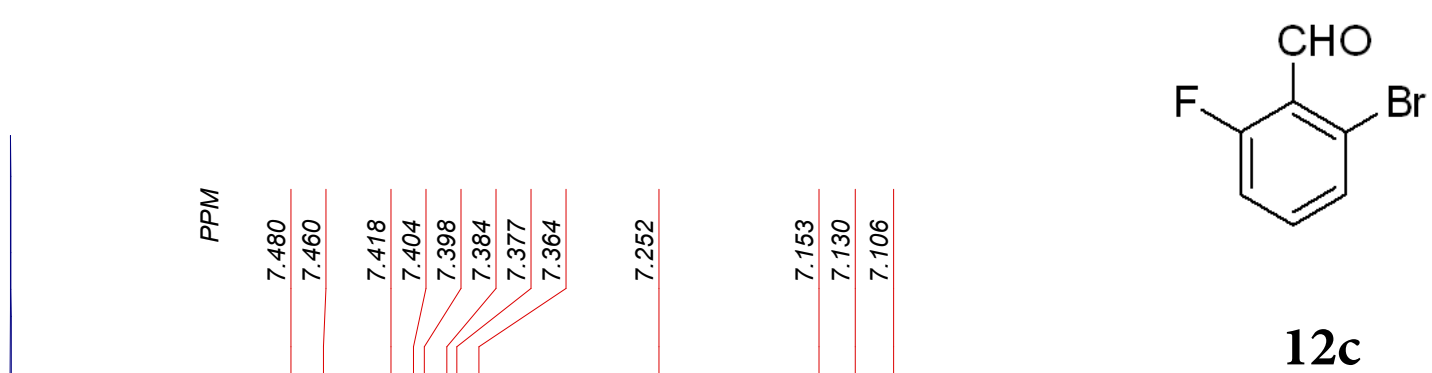

$\left|\begin{array}{l}\infty \\ \infty \\ 0\end{array}\right|$

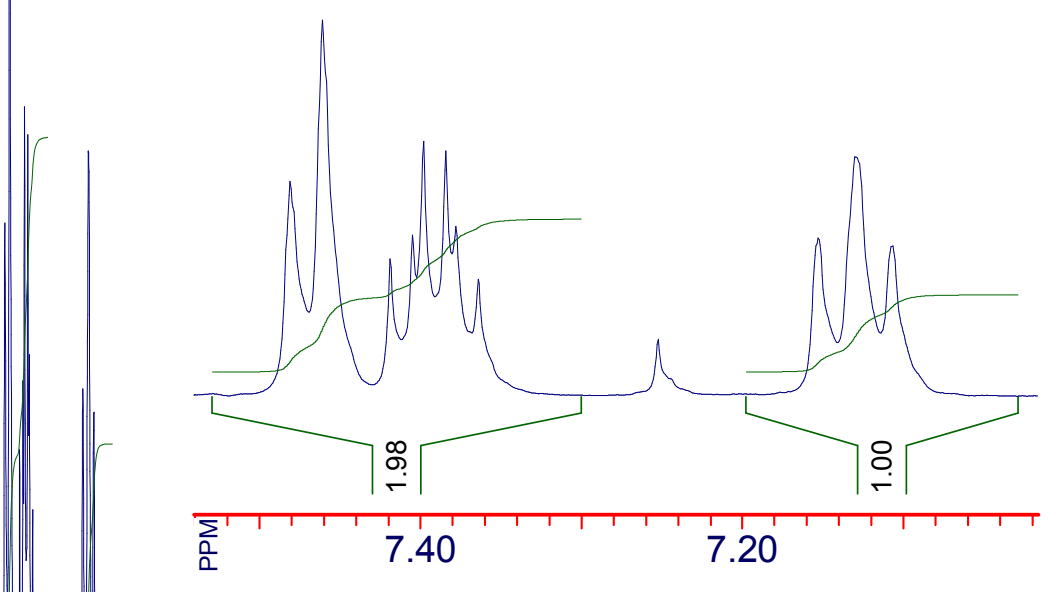

$12 \mathrm{c}$

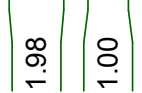

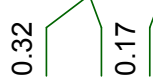

11.0

10.0

9.0

8.0

7.0

6.0

5.0

4.0

3.0

NSC: 1

PW: 0.00 usec, RG: 25

2.0

1.0 


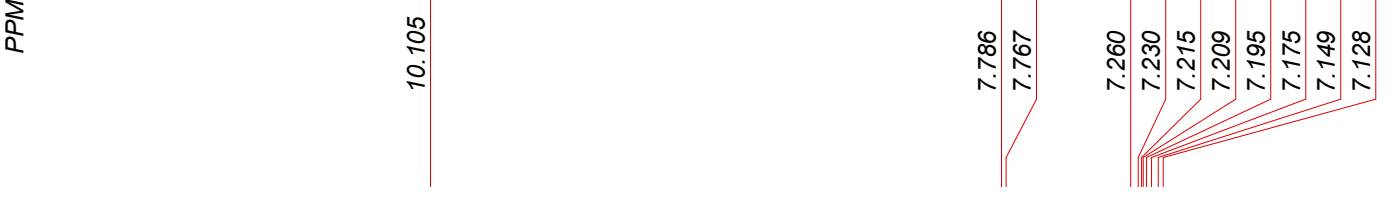

\section{PHKG016}

$\frac{10}{5}$

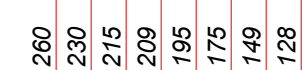

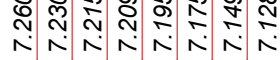

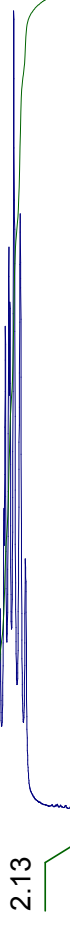

10.0

9.0

8.0

7.0

6.0

5.0

Operator: USER 1

SF: $400.1300 \mathrm{MHz}$

SW: $8224 \mathrm{~Hz}$

NSC: 1

4.0

3.0

PW: 0.00 usec, RG: 22

AQ: $1.99 \mathrm{sec}, \mathrm{RD}: 0.00 \mathrm{sec}$ 


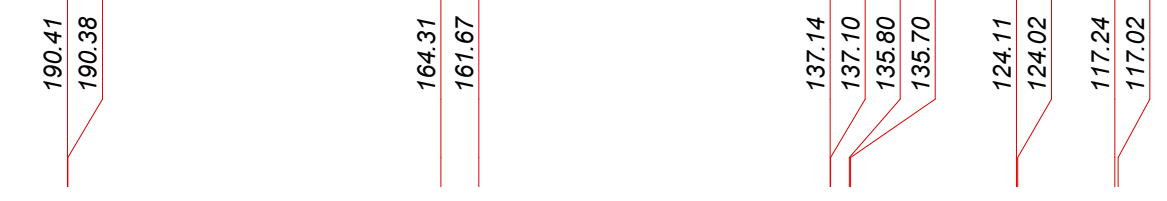

\section{PHKG016_C13}

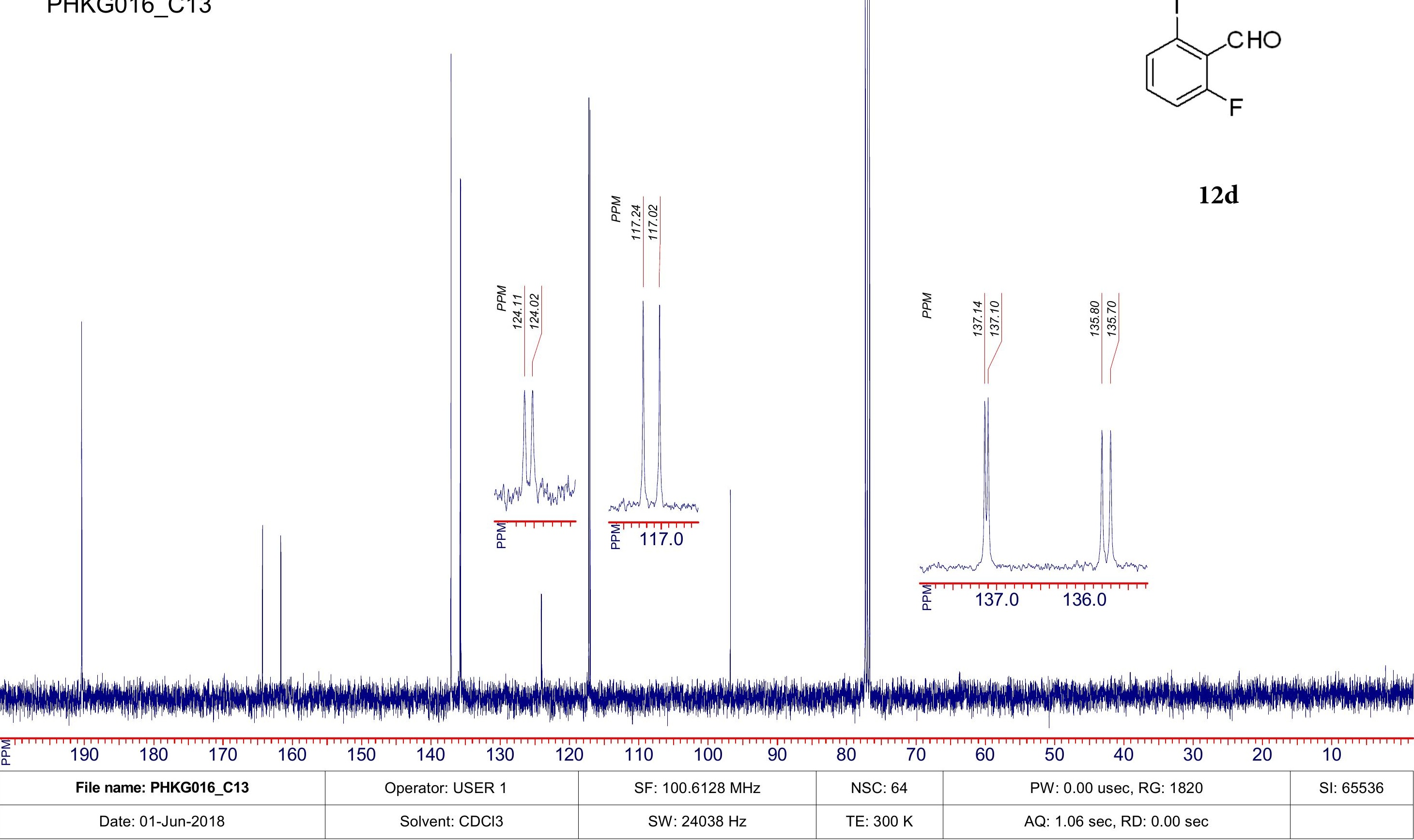




\section{PHKG016_C13APT}
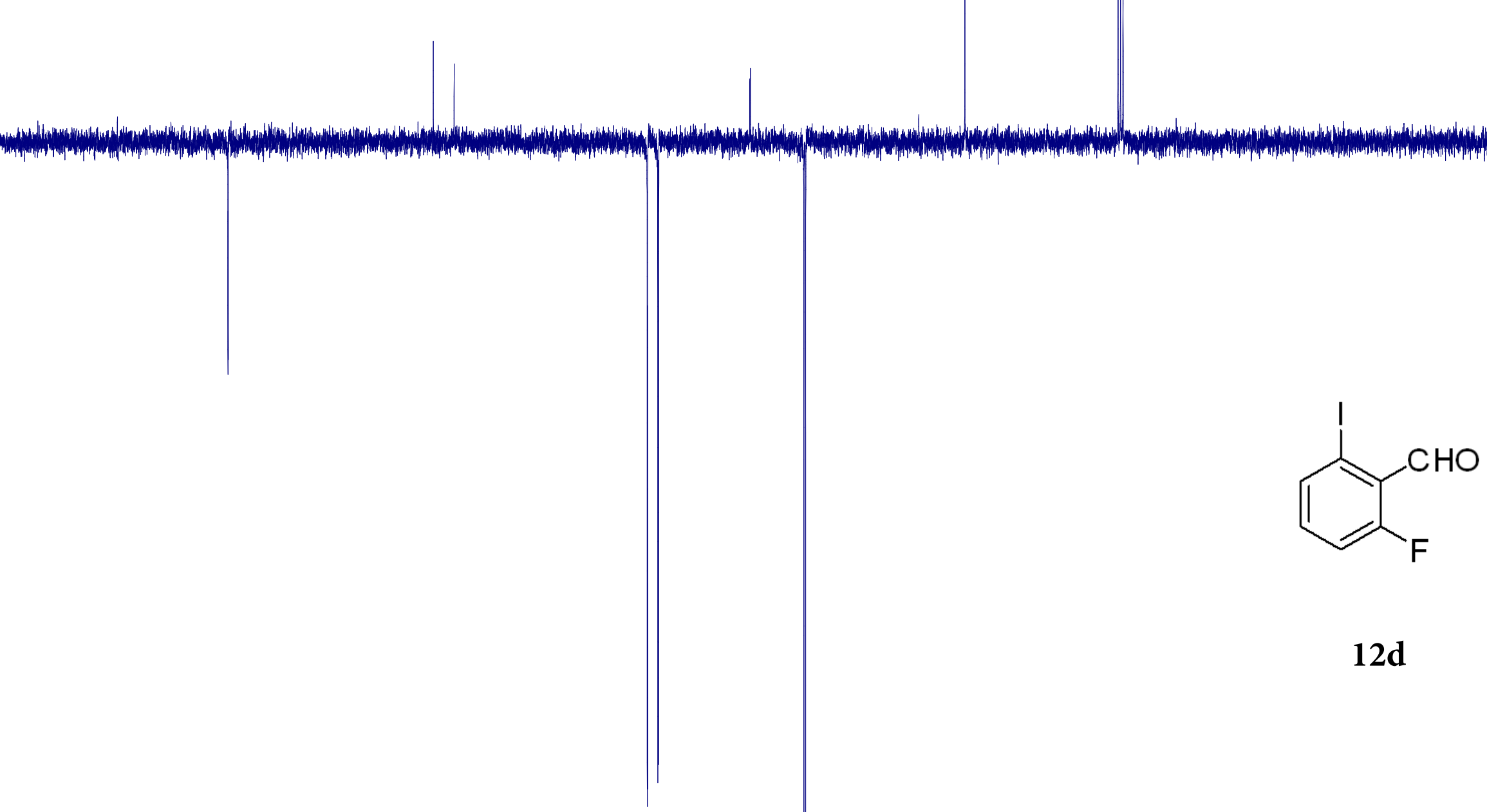

12d

$\begin{array}{ccc}200 & 180 \\ & \text { File name: PHKG016_C13APT }\end{array}$

160

140

+120

PT

0

Operator: USER 1

120

100
SF: $100.6128 \mathrm{MHz}$

80

NSC: 202

$60^{1}$

$\begin{array}{cc}40 & 20 \\ \text { PW: } 0.00 \mathrm{usec}, \mathrm{RG}: 2050 \\ \text { AQ: } 1.36 \mathrm{sec}, \mathrm{RD}: 0.00 \mathrm{sec}\end{array}$


PHKG016_F19

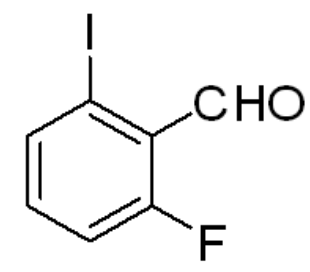

12d

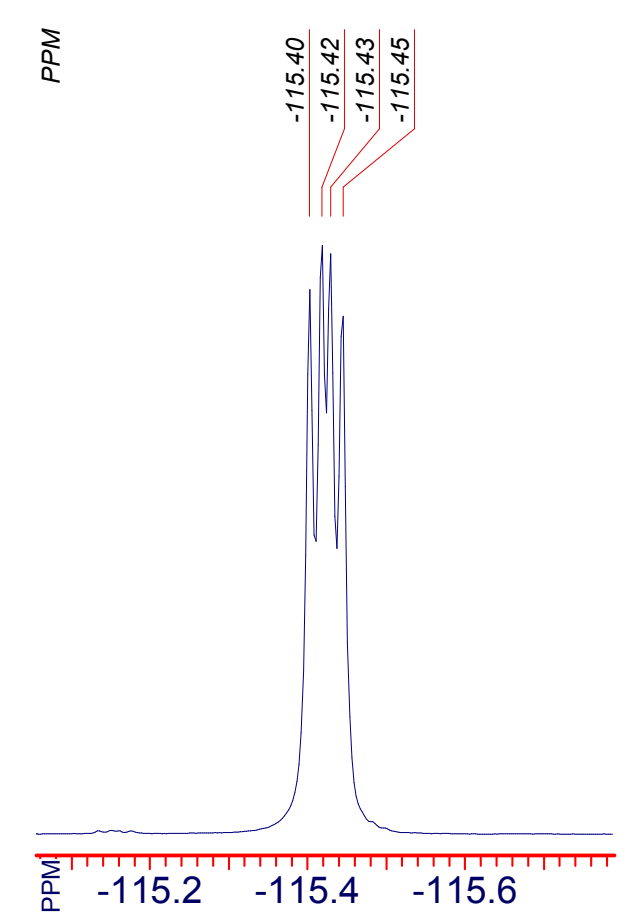

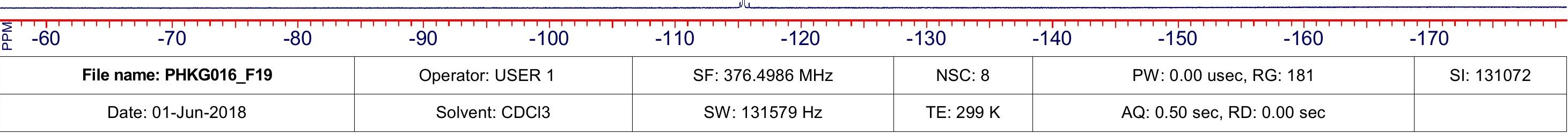




\section{PHKG008.fid}
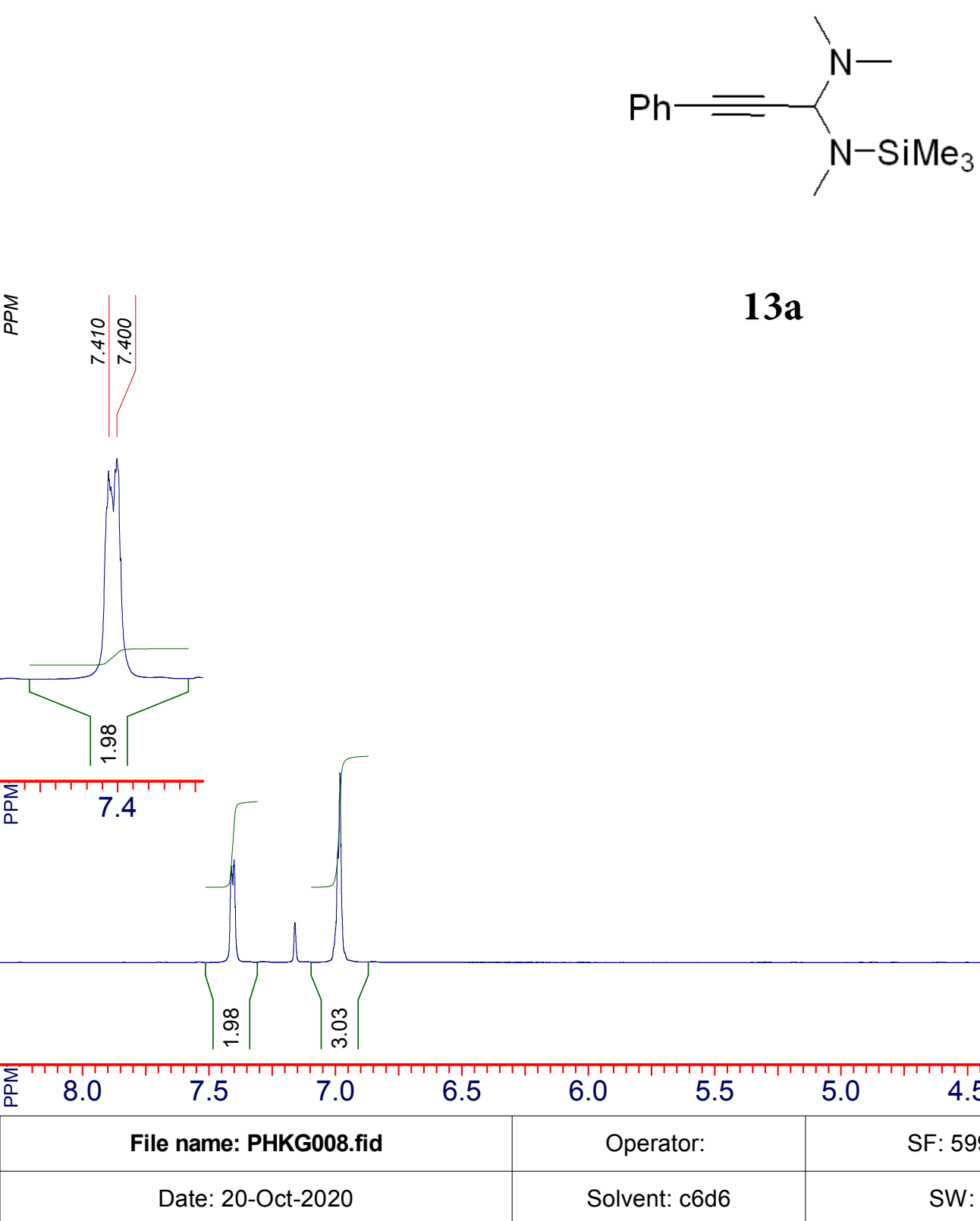

$13 a$
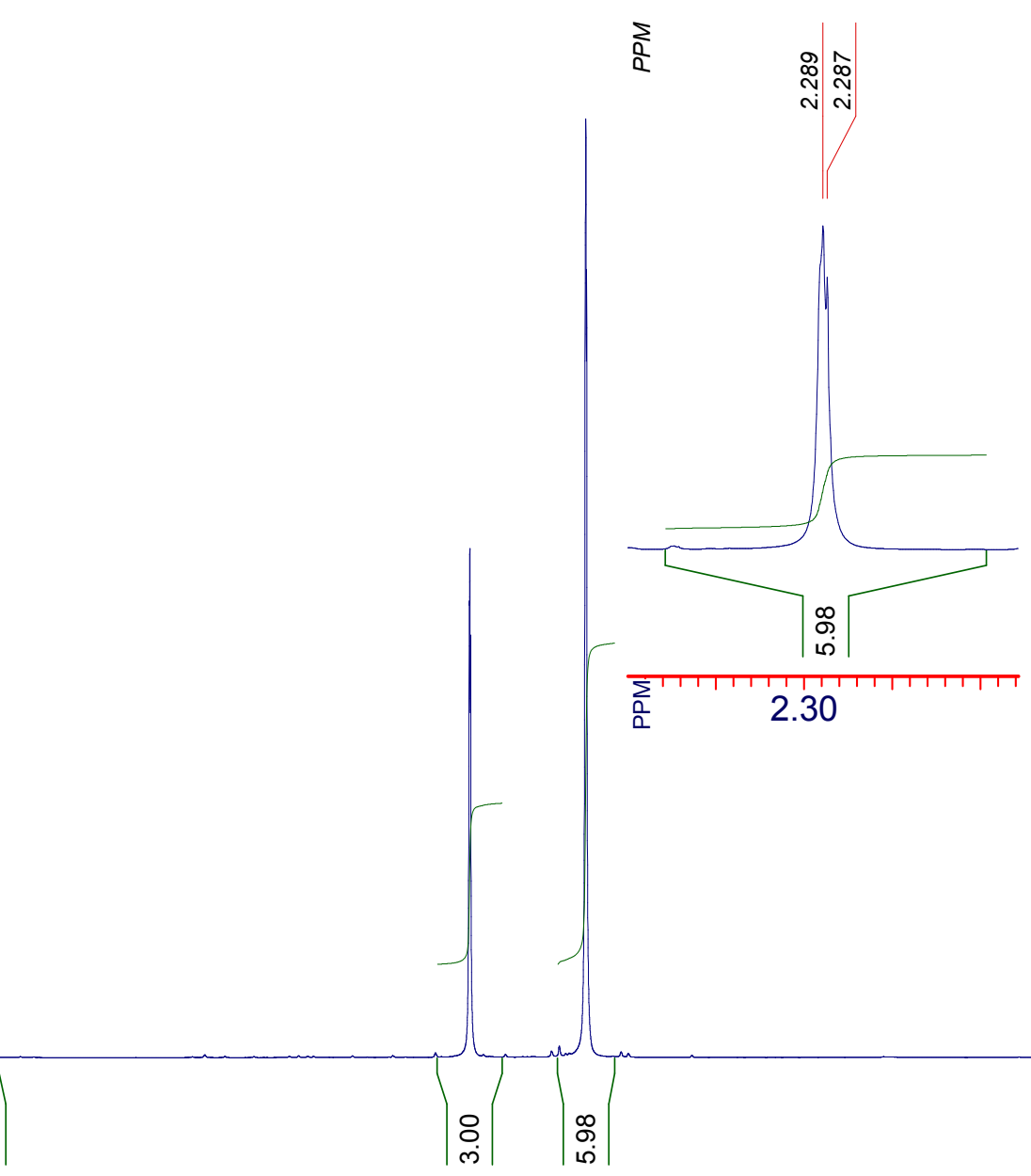

\begin{tabular}{l|l} 
Operator: & SF: $599.7780 \mathrm{MHz}$
\end{tabular}




\section{PHKG008_C13.fid}
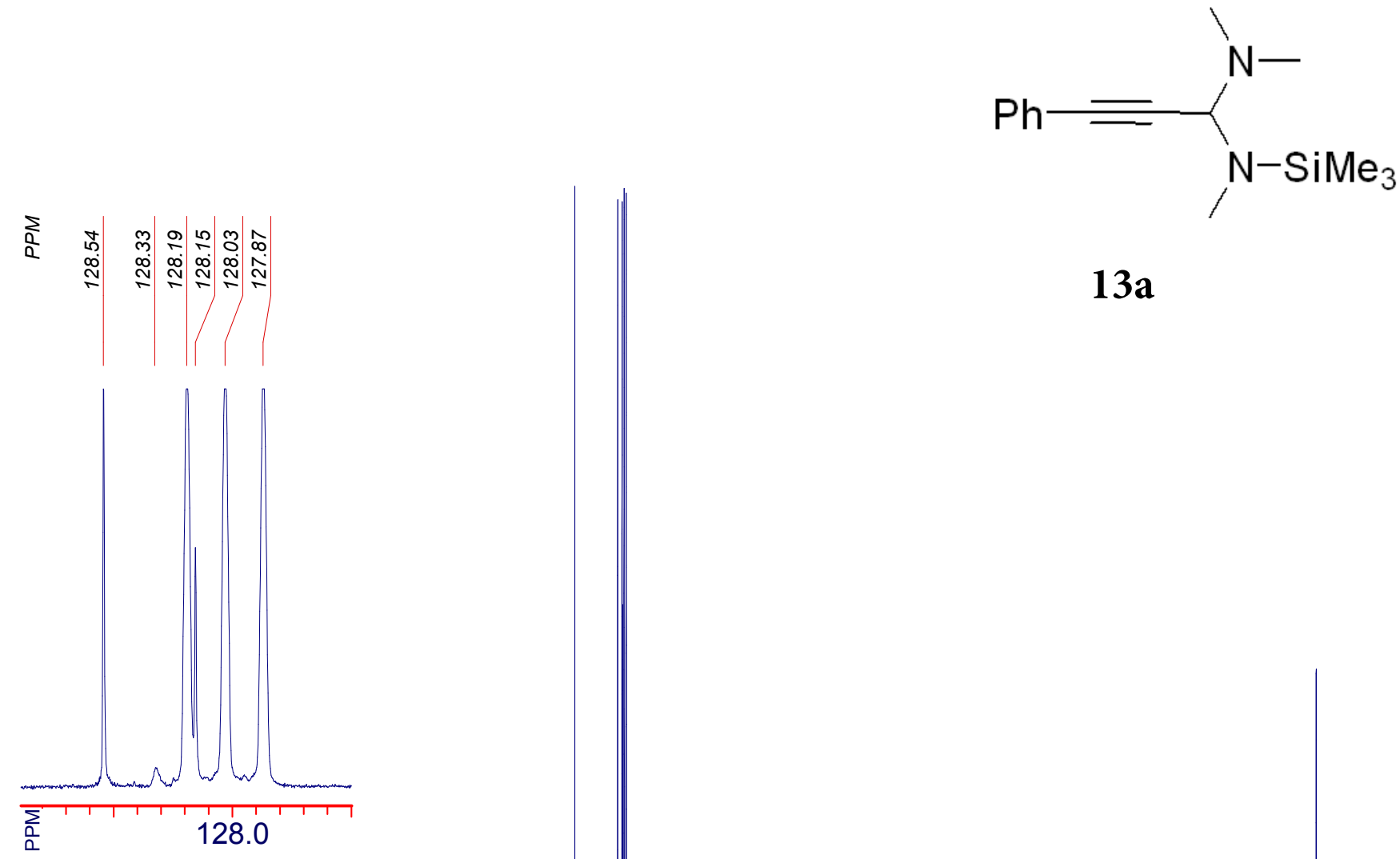

$13 a$

हागाामा1

170

160

150

140

130

120

110

00

90

Operator:

SF: $150.8297 \mathrm{MHz}$

80

70

60

50

50

40

30

20 


\section{PHKG008_C13APT.fid}
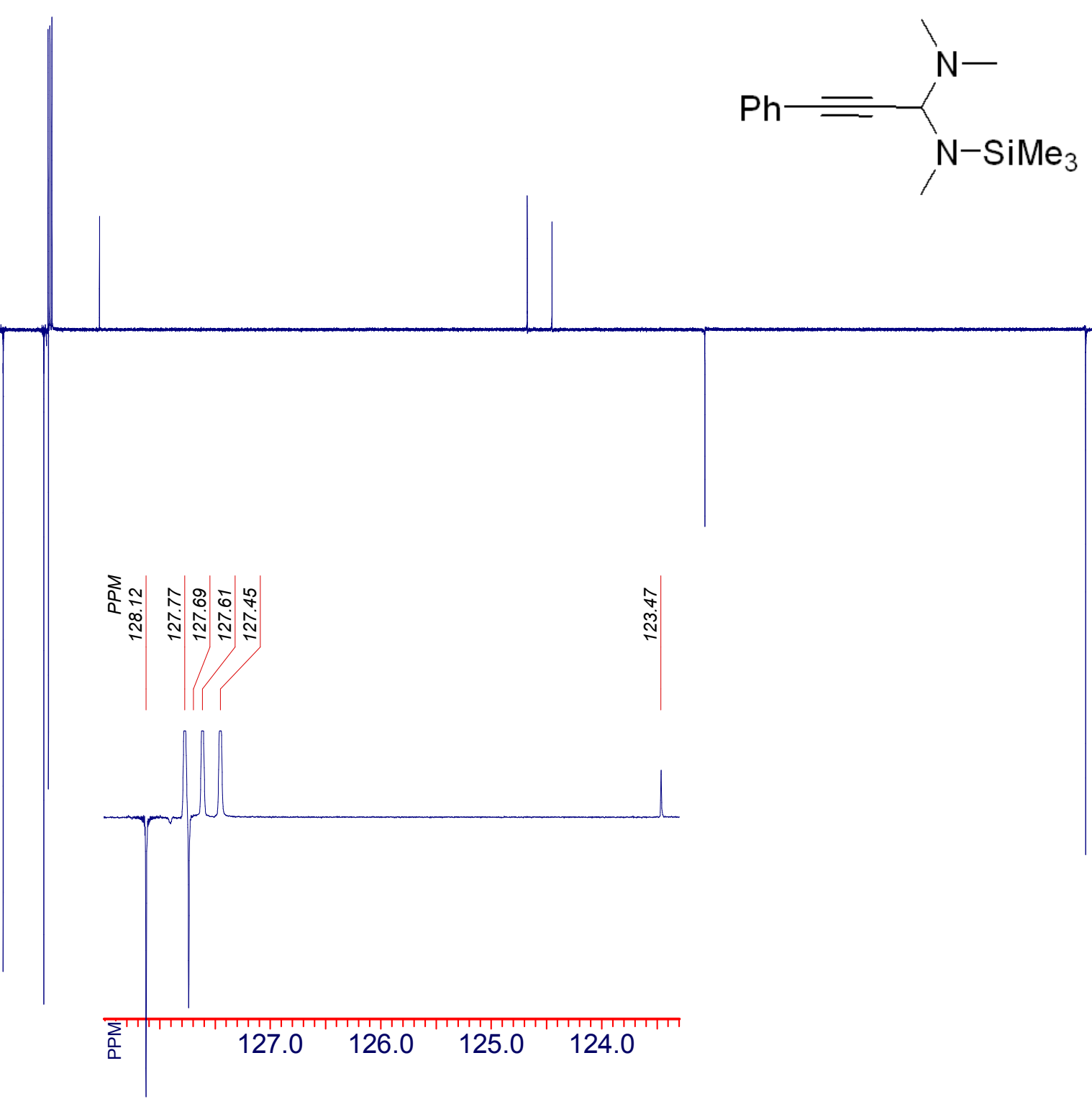

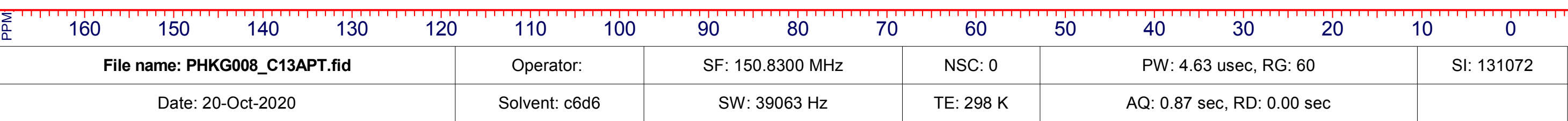


phkg012.fid

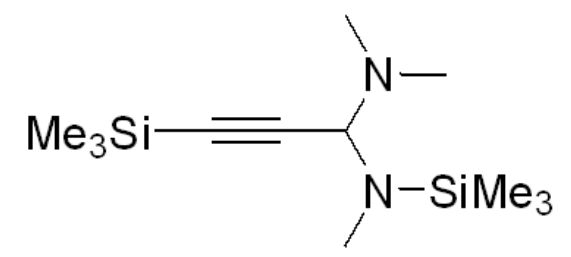

$13 b$

File name: phkg012.fid

8.0

7.0

6.0

$\left|\begin{array}{l}8 \\ \hdashline \\ i\end{array}\right|$

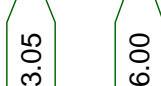




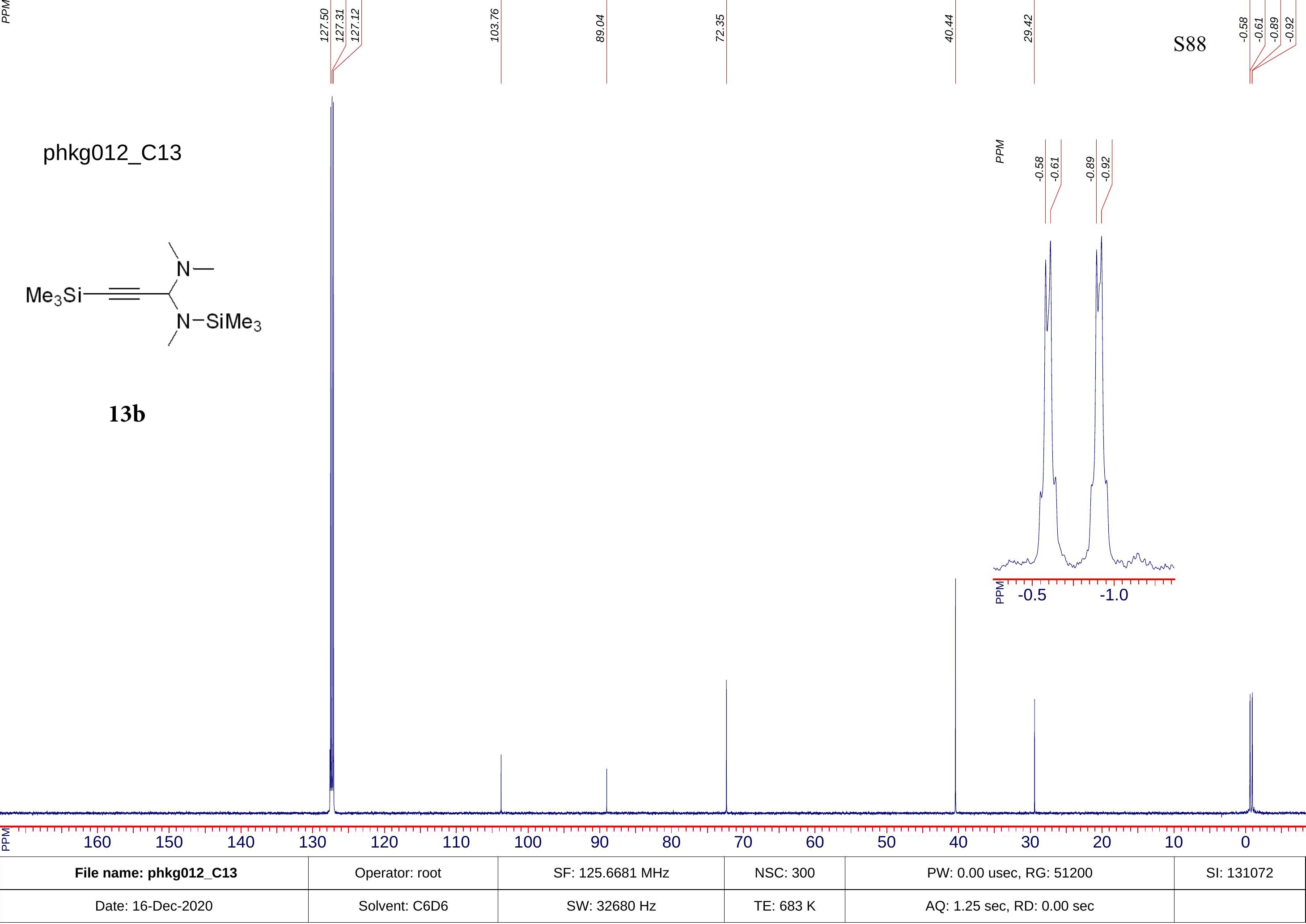




\section{phkg012_C13APT}<smiles>C[SiH2]C#CC(N(C)C)N(C)C</smiles>

\begin{tabular}{|c|c|c|c|c|c|c|c|}
\hline 240 & 200 & 160 & 140 & 100 & 40 & 20 & 0 \\
\hline & File name: phkg012_C13APT & Operator: root & SF: $125.6681 \mathrm{MHz}$ & NSC: 144 & PW: 0.00 usec, RG: 51200 & & SI: 65536 \\
\hline & Date: 16-Dec-2020 & Solvent: C6D6 & SW: $32680 \mathrm{~Hz}$ & TE: $683 \mathrm{~K}$ & AQ: $1.57 \mathrm{sec}, \mathrm{RD}: 0.00 \mathrm{sec}$ & & \\
\hline
\end{tabular}





\section{PHKG044_C13}<smiles>CC(=O)C#CC(N(C)C)N(C)[SiH3]</smiles>

\section{$13 c$}

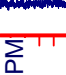

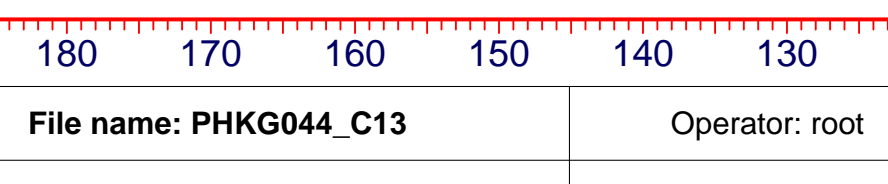

$120110 \quad 100$




\section{PHkg012.fid}

13d
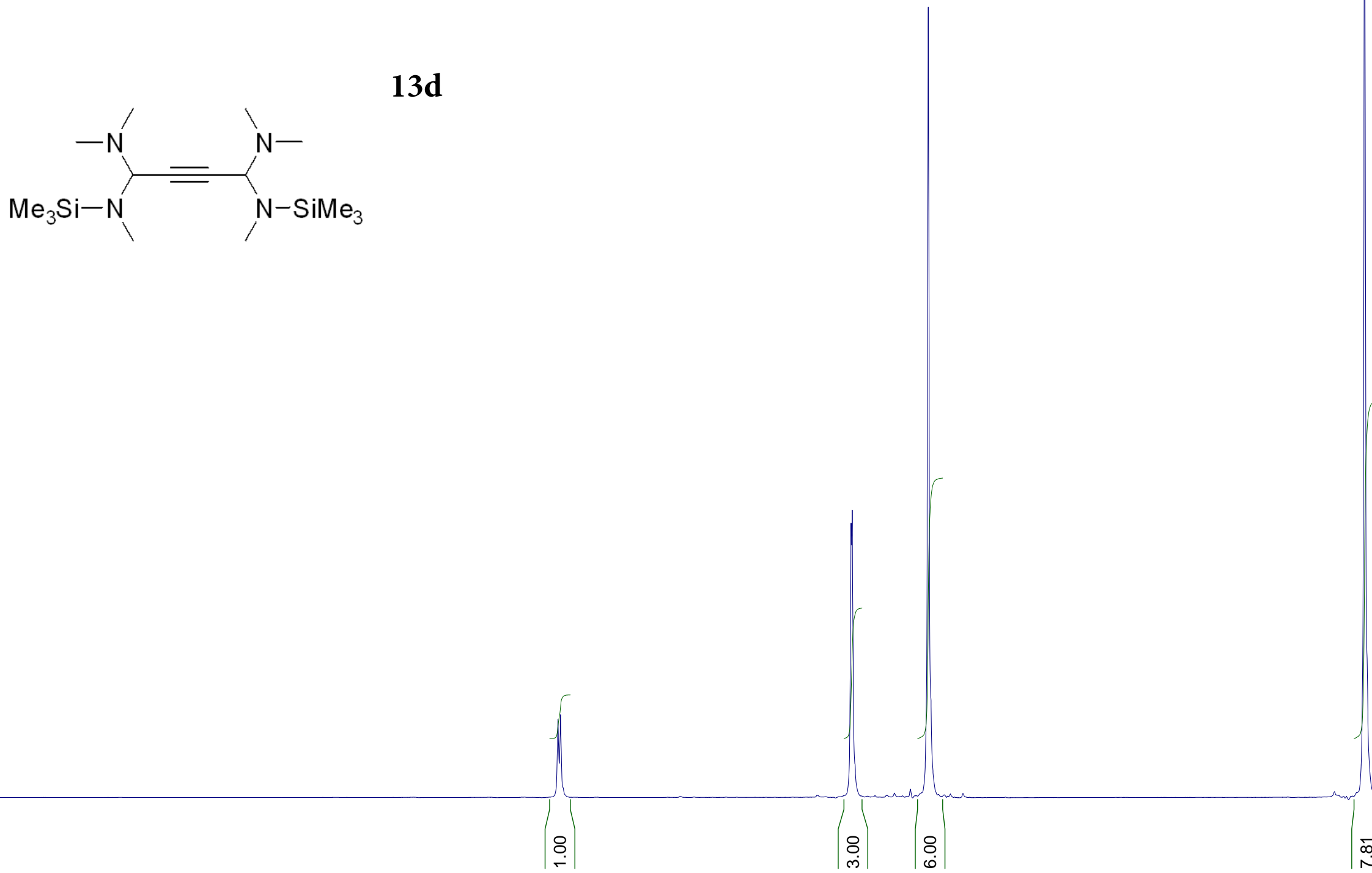

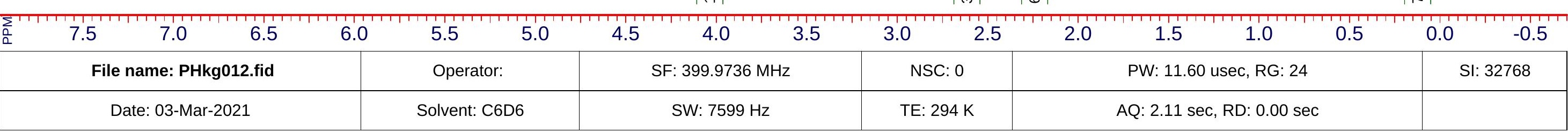




\section{phkg012_C13}

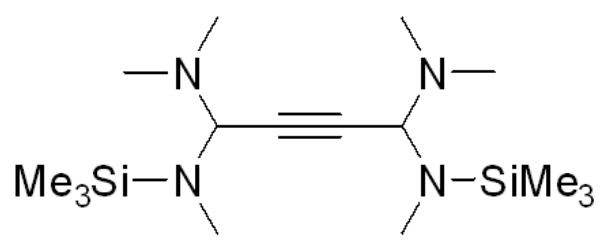

13d

\section{$13 d$}

$\sum_{n=11}^{1+n}$

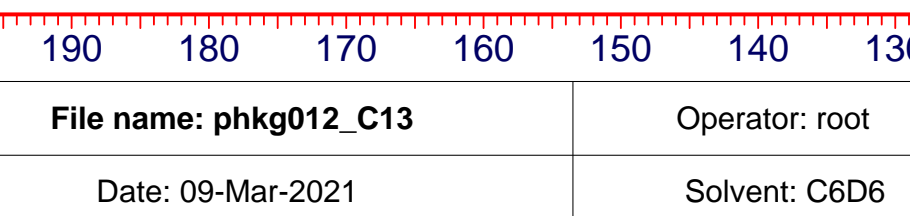

$130 \quad 120$

$110 \quad 100 \quad 90$

$80 \quad 70$

$60 \quad 50 \quad 40$

30

$20 \quad 10$

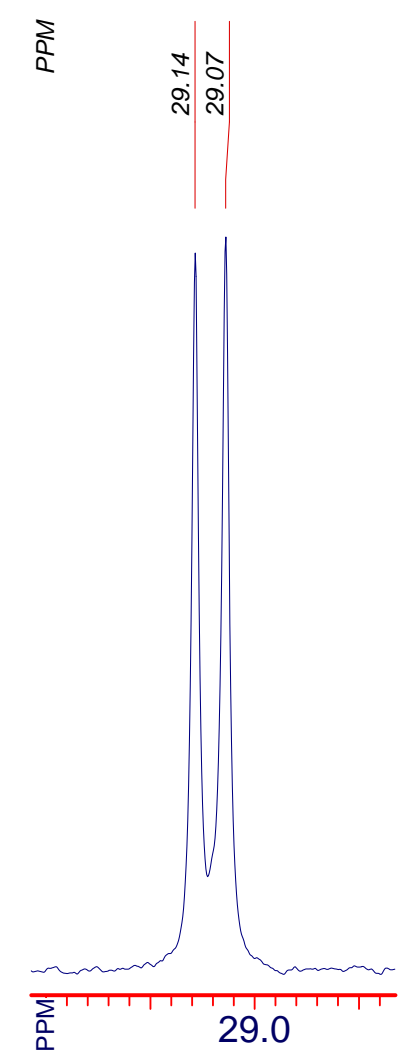

NSC: 562

PW: 0.00 usec, RG: 51200

SI: 131072 


\section{phkg012_C13APT}

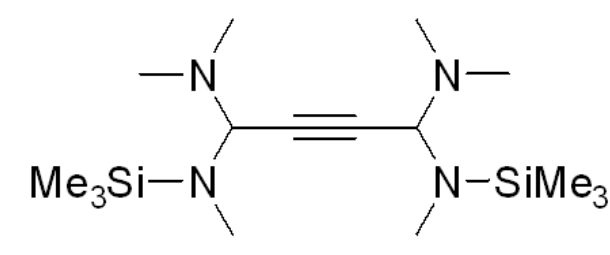

13d

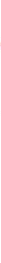



Unknown Spectrum based on Apex
Abundance

8000
6000
4000
2000

$\mathrm{m} / \mathrm{z-->}$

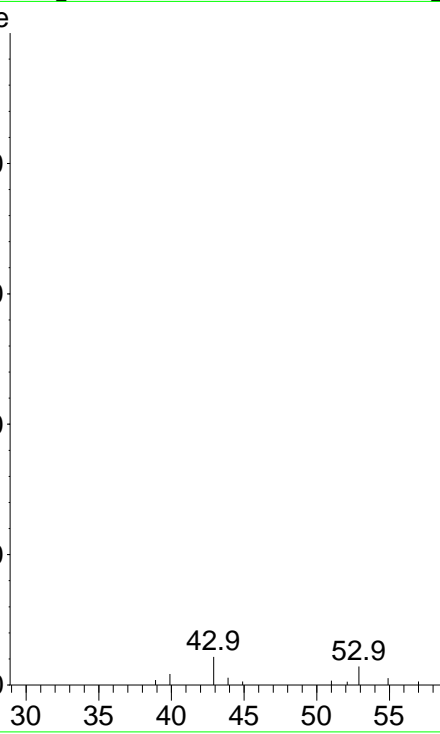

\section{- Chemstation Integrator}

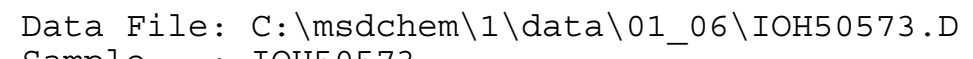

Sample : IOH50573

Peak Number: 1 at $5.706 \mathrm{~min}$ Area: 1011293 Area \% 1.83

The 3 best hits from each library. Ref $\backslash \#$ CAS $\backslash \#$ Qual

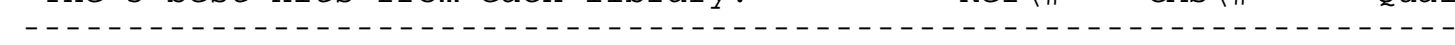

C: \Database $\backslash$ EMPTY.L No hits were retrieved.

Scan 715 (5.708 min): IOH50573.Dldata.ms

104.9

\section{0}

90.8 $100 \quad 105 \quad 110 \quad 115 \quad 120$

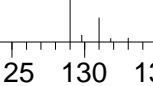

143.0

\section{$140 \quad 145$}
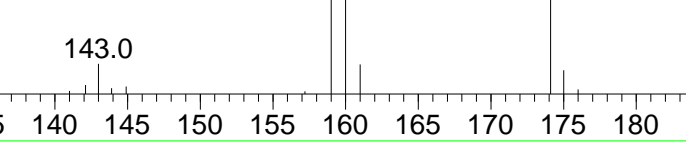

.

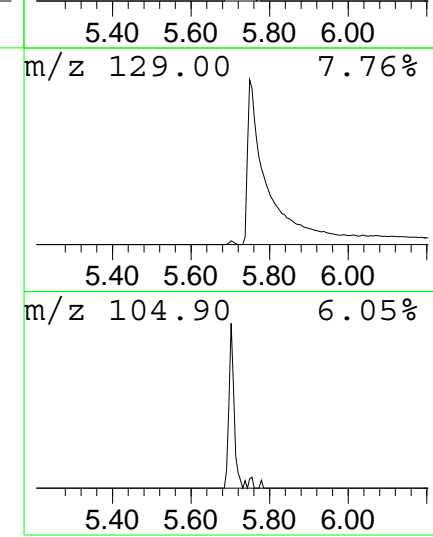




\section{PHKG005_C13APT}

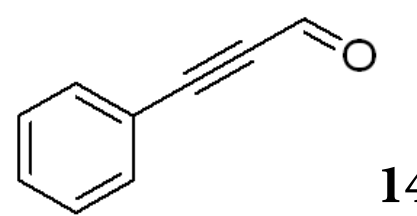

14

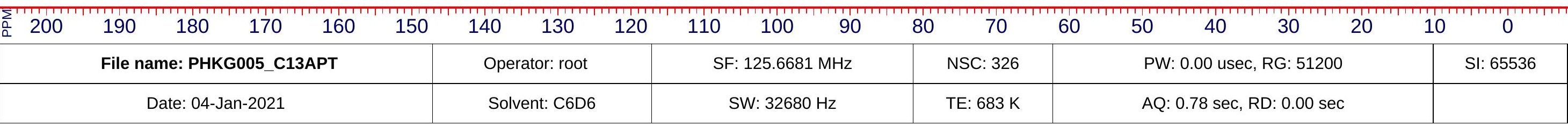




\section{phkg003_C13}<smiles>CN=CN(SC)c1ccccc1</smiles> 


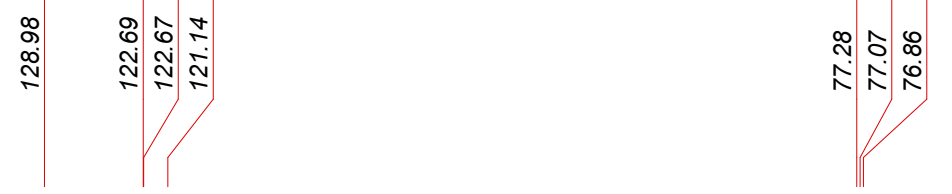

\section{phkg007_C13APT.fid}

$$
\mathrm{Ph}_{\mathrm{H}} \curvearrowright \mathrm{N}^{-\mathrm{Me}}
$$

$15^{\prime}$

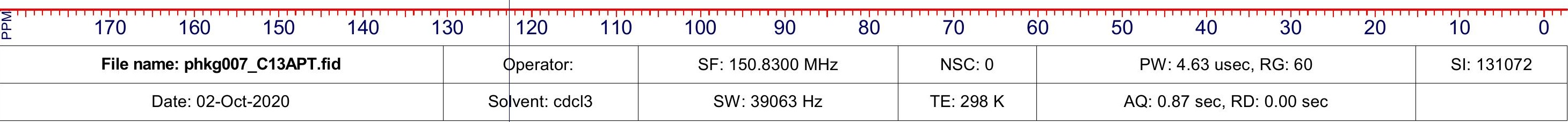




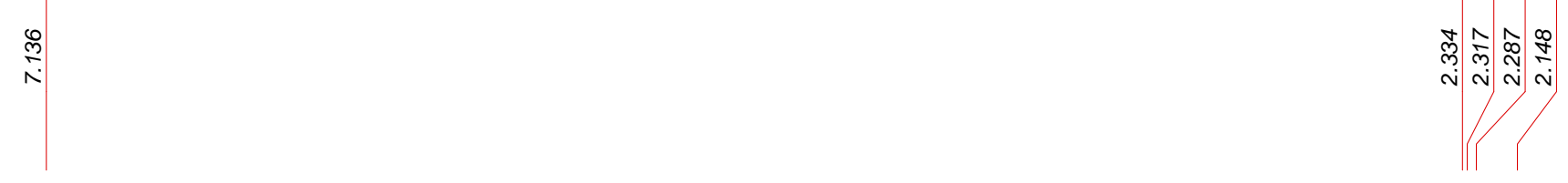

PHkg017.fid
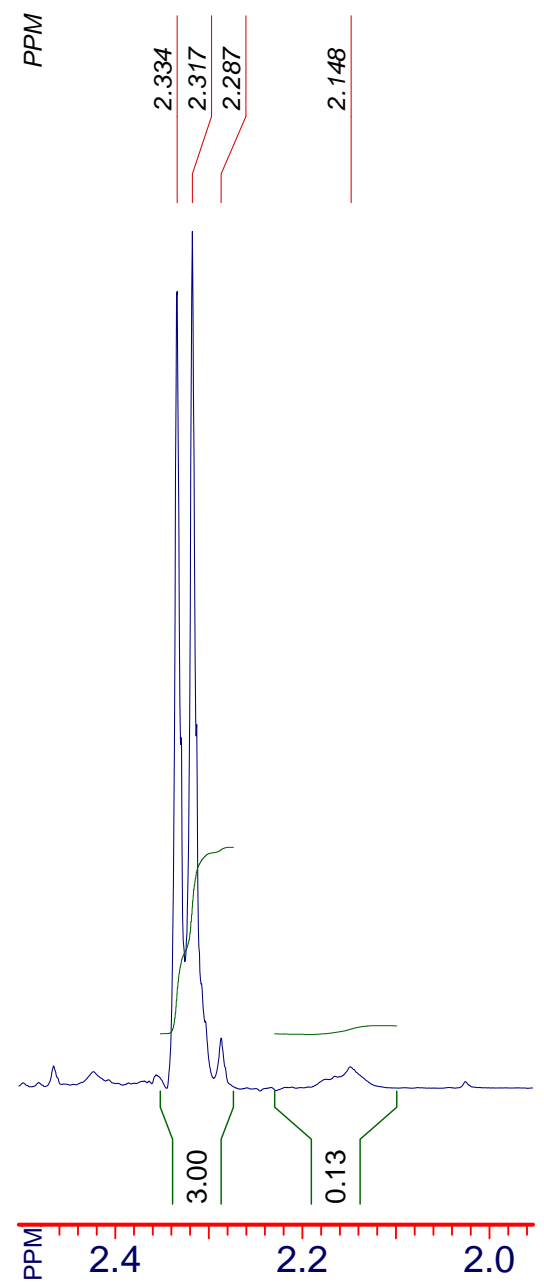

$\mathrm{Me}_{3} \mathrm{Si}-\mathrm{NH}$

\section{$16 a$}

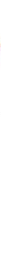



phkg018 fid

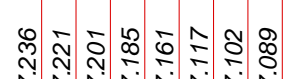

象fid

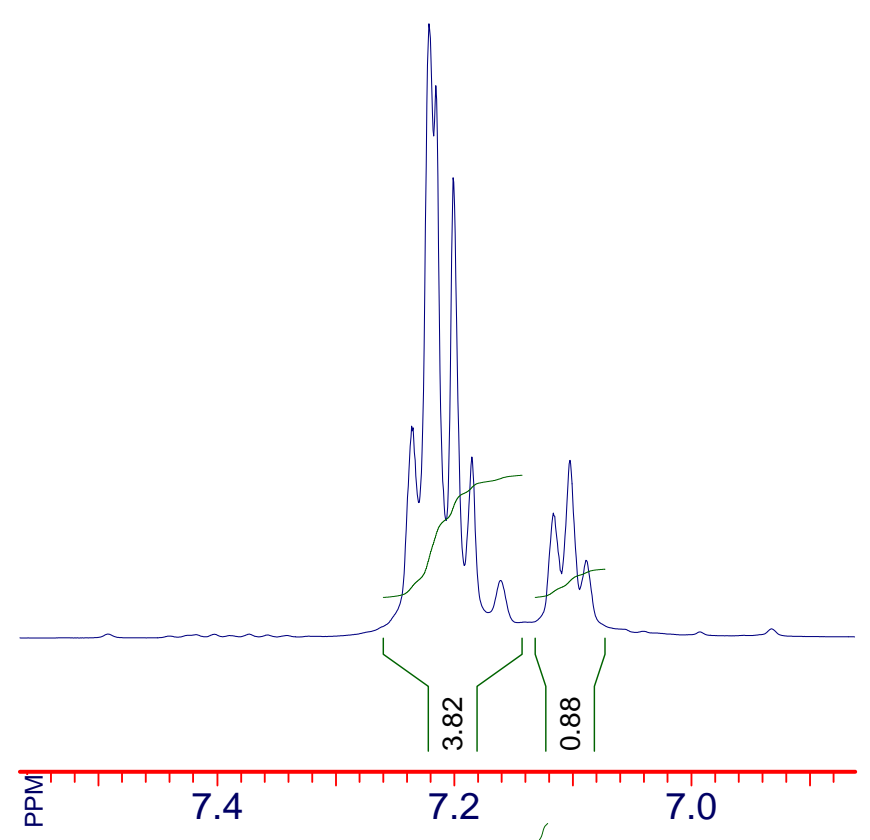

$\sum_{0}^{\infty}$
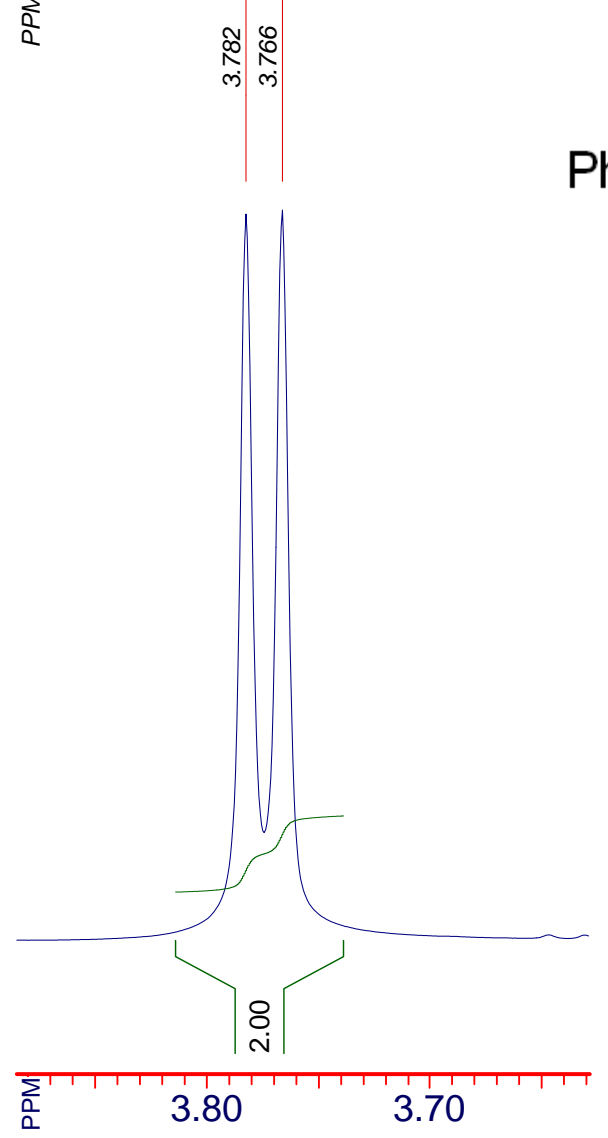

$\mathrm{SiMe}_{3}$

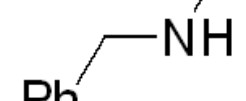

$16 b$

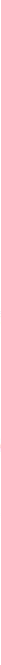


phkg018_C13.fid

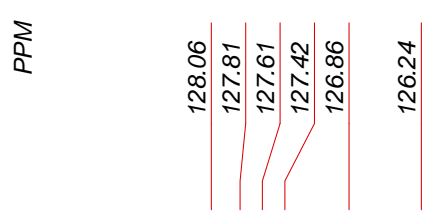

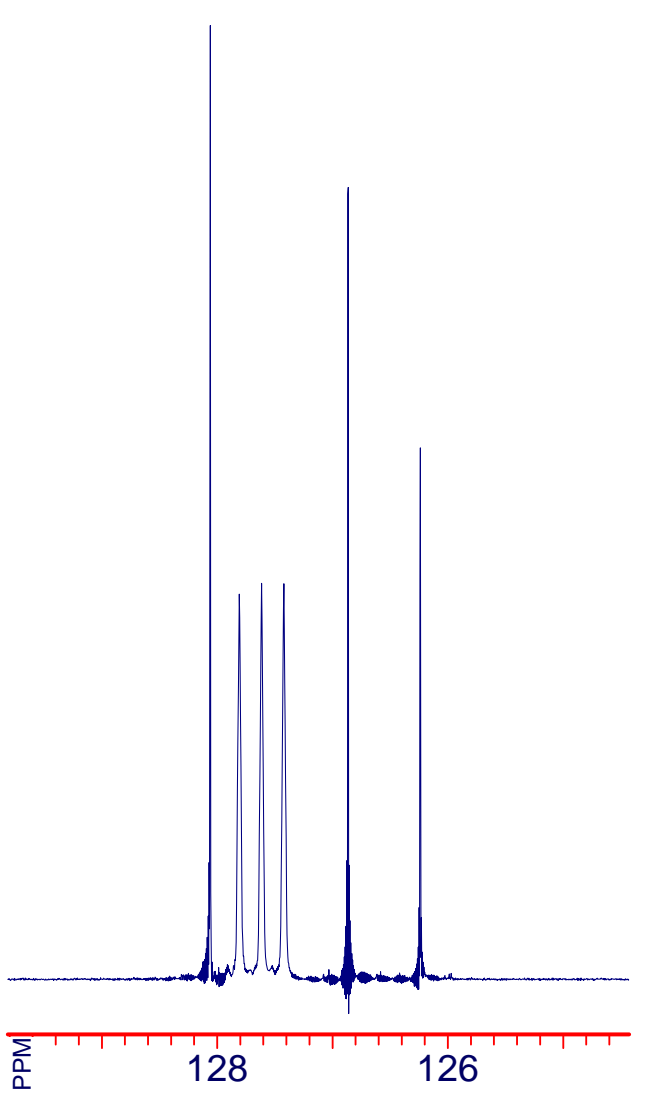

$\mathrm{SiMe}_{3}$

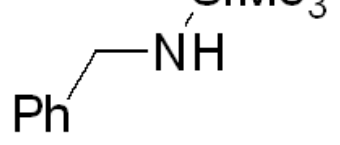

$16 b$

$\sum_{0=11}^{111}$

$\begin{array}{lllll}170 & 160 & 150 & 140 & 130\end{array}$

$\begin{array}{lllll}120 & 110 & 100 & 90 & 80\end{array}$

$80 \quad 70 \quad 60 \quad 50$

Operator:

SF: $125.6921 \mathrm{MHz}$

NSC: 0

SW: $31250 \mathrm{~Hz}$

TE: $298 \mathrm{~K}$

$\begin{array}{llll}50 & 40 & 30 & 20\end{array}$

20 10 PW: 3.27 usec, RG: 60 AQ: $1.02 \mathrm{sec}, \mathrm{RD}: 0.00 \mathrm{sec}$ 


\section{phkg018_C13APT.fid}

$\mathrm{SiMe}_{3}$<smiles>[AlH2]NCc1ccccc1</smiles>

$16 b$

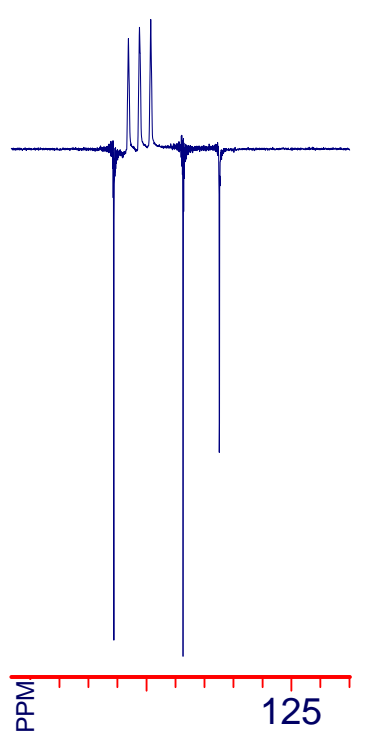

200

180

160

$140 \quad 120$

120

100

80

60

60

PW: 5.00 usec, RG: 60 40

20

0

File name: phkg018_C13APT.fid

Operator:
SF: $125.6907 \mathrm{MHz}$ NSC: 0 



\section{PHKG005_C13}<smiles>CN=C(N(C)C)C(Cl)(Cl)Cl</smiles>

$17 \mathbf{a}$

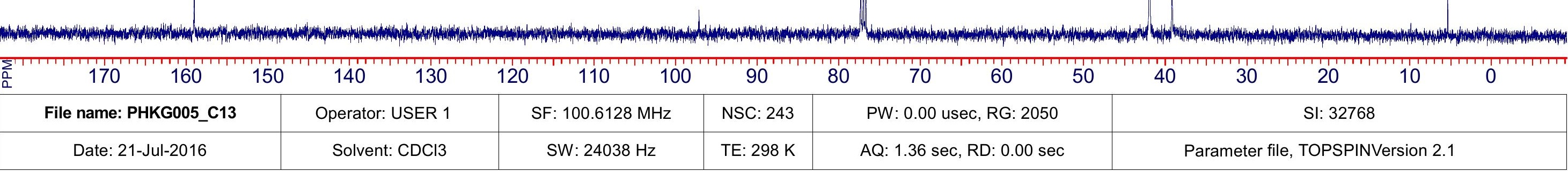




\section{phkg002.fid}

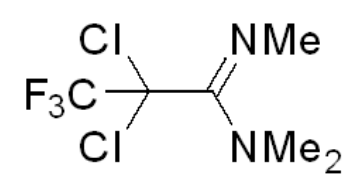

17b
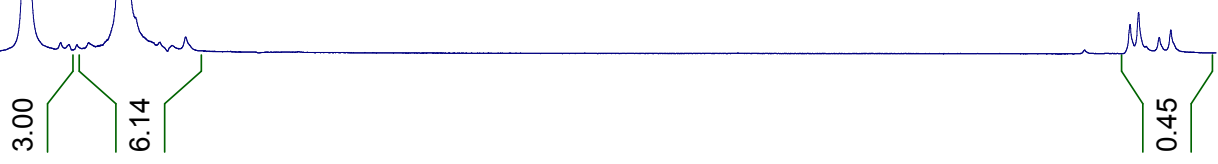

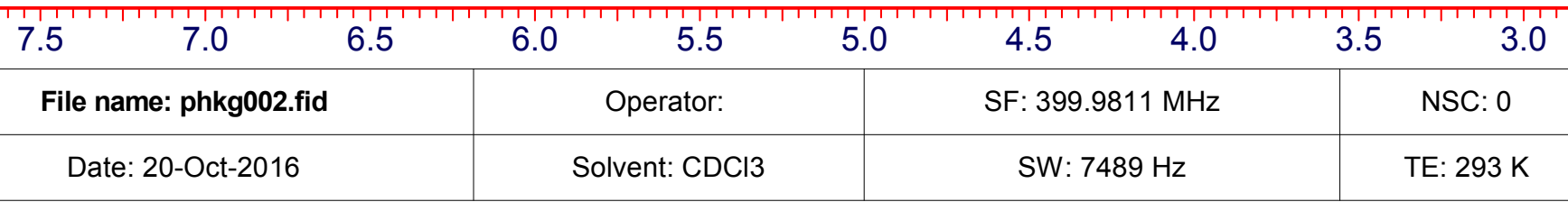

2.5

2.0

1.5

PW: 7.50 usec, RG: 30

1.0

0.5

$A Q: 1.20 \mathrm{sec}, \mathrm{RD}: 0.00 \mathrm{sec}$ 


\section{PHkg002_C13}<smiles>CN=C(N(C)C)C(Cl)(Cl)C(F)(F)F</smiles>

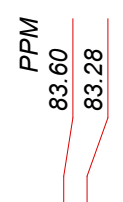

총

17b

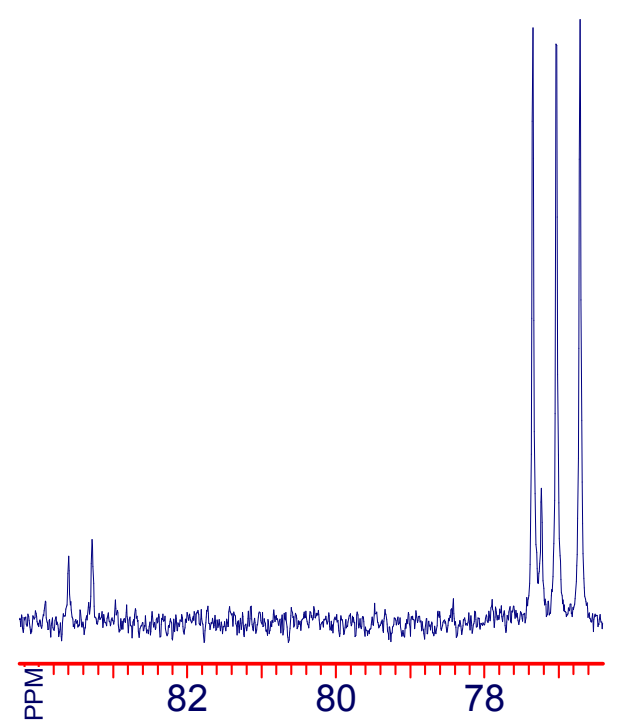




\section{PHkg002_F19(H1)}

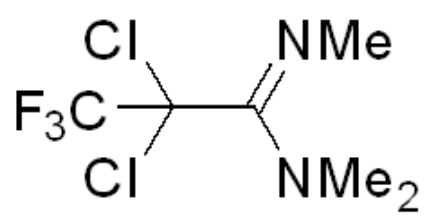

17b

$\begin{array}{lll}\sum_{0} & 0 & -10\end{array}$

File name: PHkg002_F19(H1) Date: 20-Oct-2016
$20-30 \quad-40 \quad-50$

$-50 \quad-60$

$1+1+1+1+119$
-60

Operator: USER 1

SF: $376.4986 \mathrm{MHz}$

\begin{abstract}
70
\end{abstract}

NSC: 4

Solvent: $\mathrm{CDCl} 3$

SI: 262144 


\section{PHKG012_C13}<smiles>CN=C(N(C)C)C(Cl)(Cl)C(=NC)N(C)C</smiles>

$18 a$

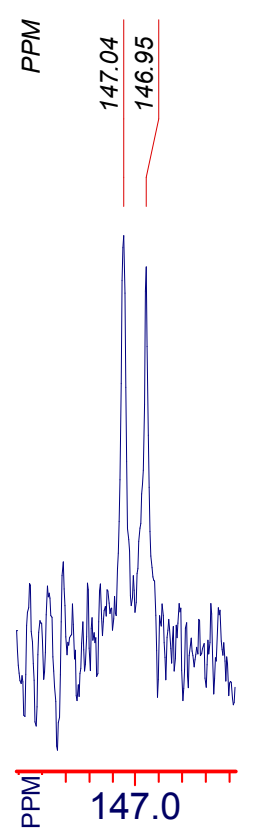

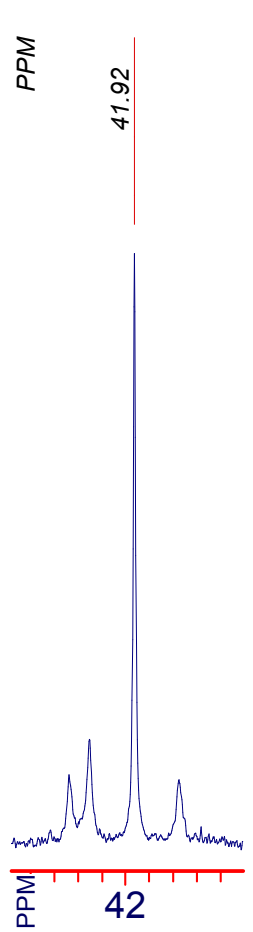

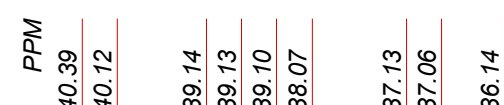

वें कें कें कें
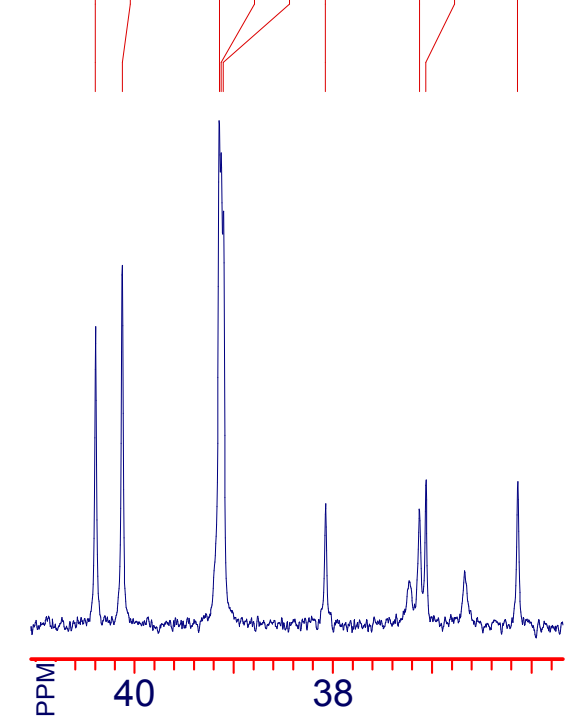

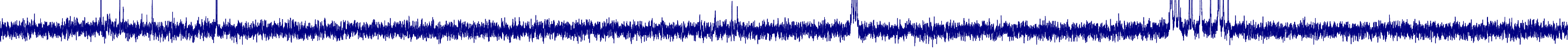

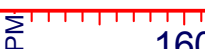
150

140

130

120

110

100

90

80

70

60

50

Operator: root

SF: $125.7125 \mathrm{MHz}$

NSC: 310

PW: 0.00 usec, RG: 51200

20 


\section{PHKG012_C13APT}<smiles>CN=C(N(C)C)C(Cl)(Cl)C(=NC)N(C)C</smiles>

\section{$18 \mathbf{a}$}

90

80

Operator: root

SF: $125.7125 \mathrm{MHz}$

70

NSC: 438

60

50

40

30

PW: 0.00 usec, RG: 51200

TE: $683 \mathrm{~K}$

AQ: $1.25 \mathrm{sec}, \mathrm{RD}: 0.00 \mathrm{sec}$

20 


\section{PHKG004.fid}

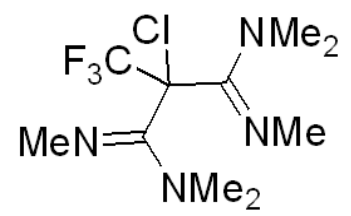

$18 b$

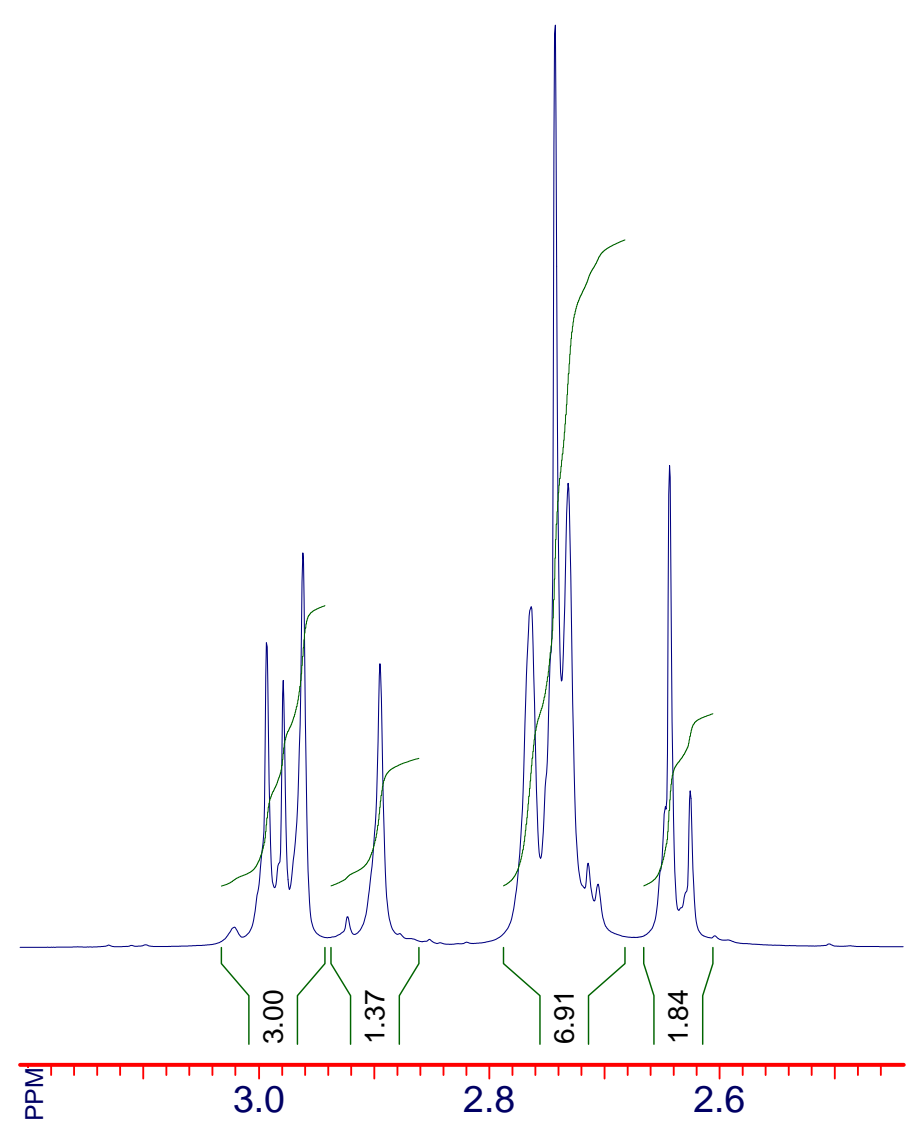

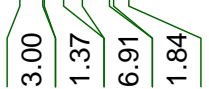

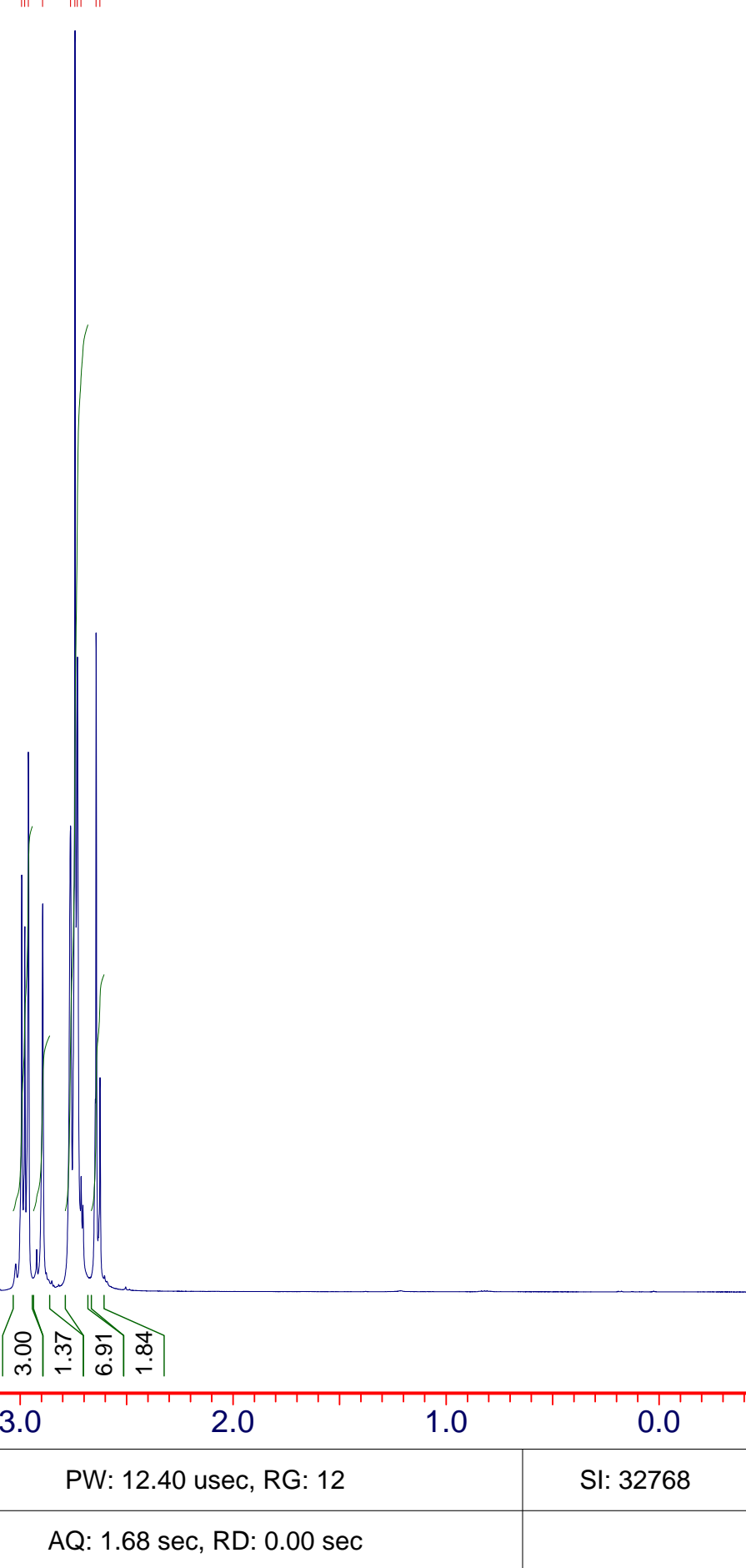




\section{PHKG004_C13APT}

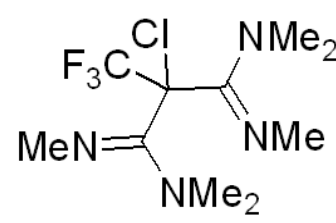

$18 b$

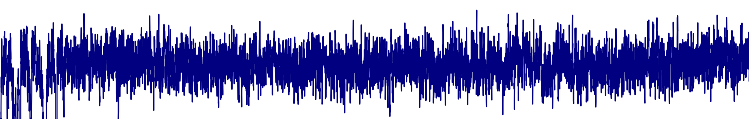$$
170
$$

60150

140

80

60

Operator: root

50

$$
40
$$

PW: 0.00 usec, RG: 51200 AQ: $1.57 \mathrm{sec}, \mathrm{RD}: 0.00 \mathrm{sec}$ 


\section{PHKG004_F19.fid}<smiles>CN=C(C)C(Cl)(C(N)=NC)C(F)(F)F</smiles>

$18 b$

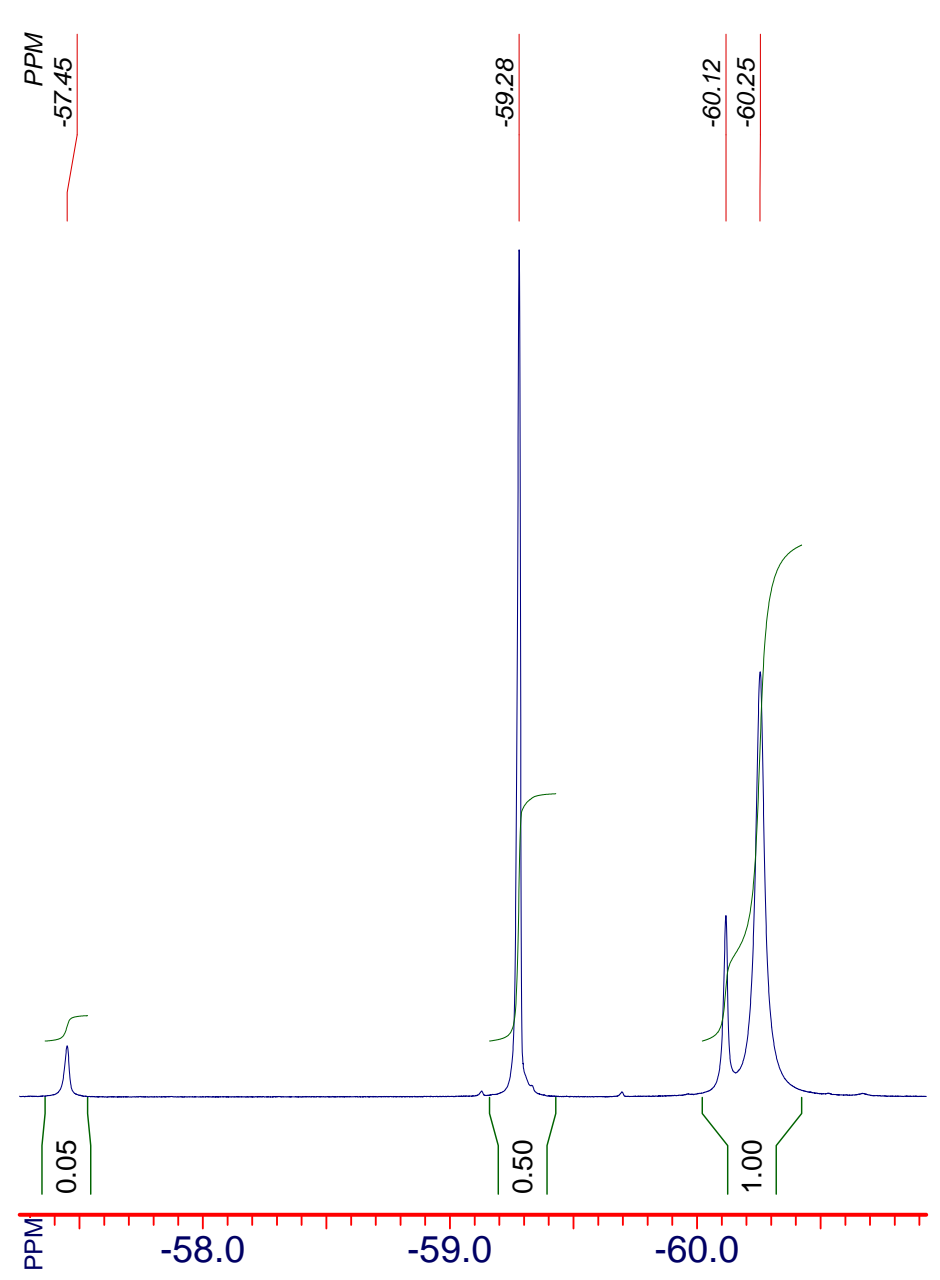

\section{$\left|\begin{array}{ccc}0 & 0 & 0 \\ 0 & 0 & 0 \\ 0 & 0 & 0 \\ 0\end{array}\right|$}

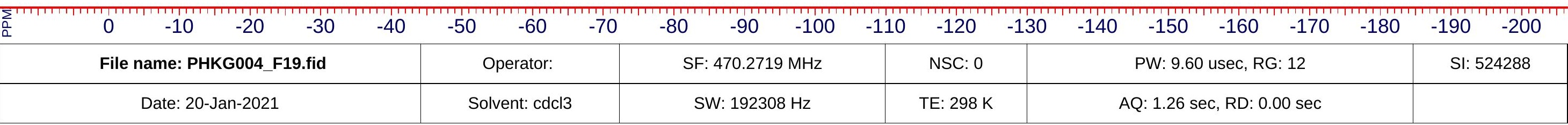




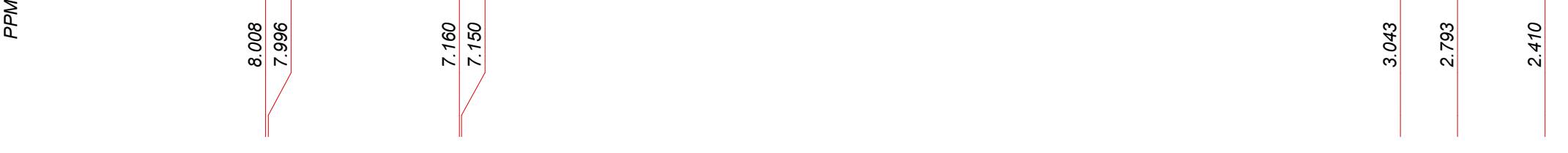

\section{phkg014.fid}

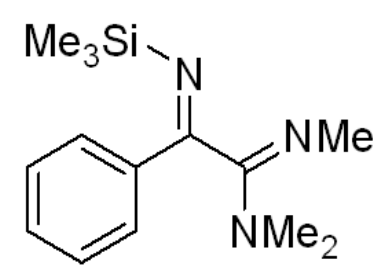

19

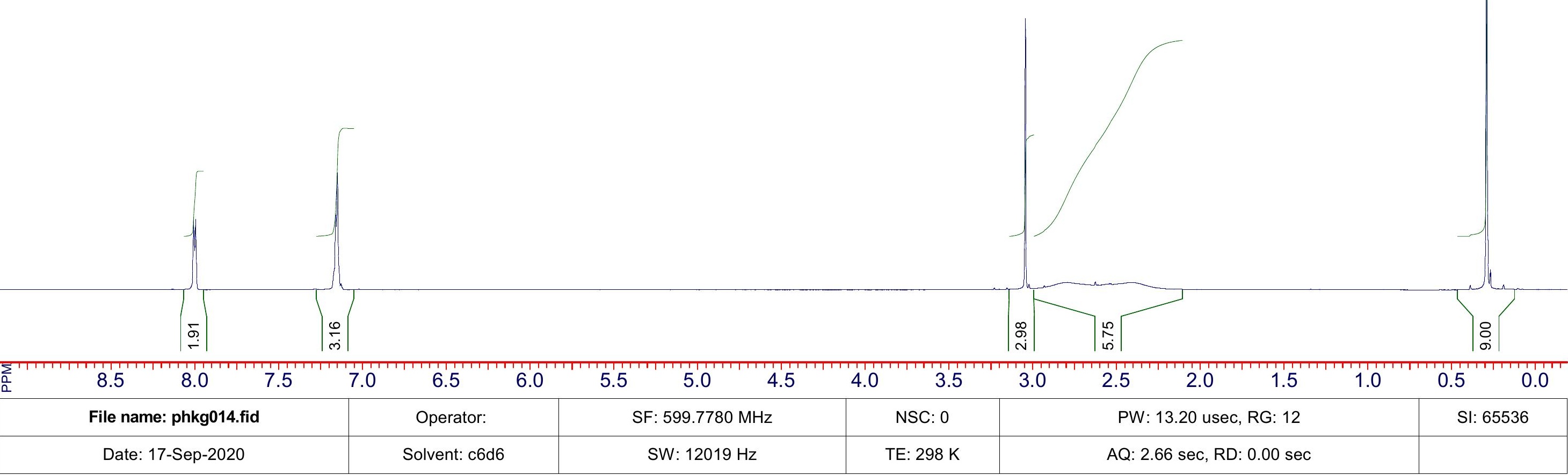


phkg014_C13.fid

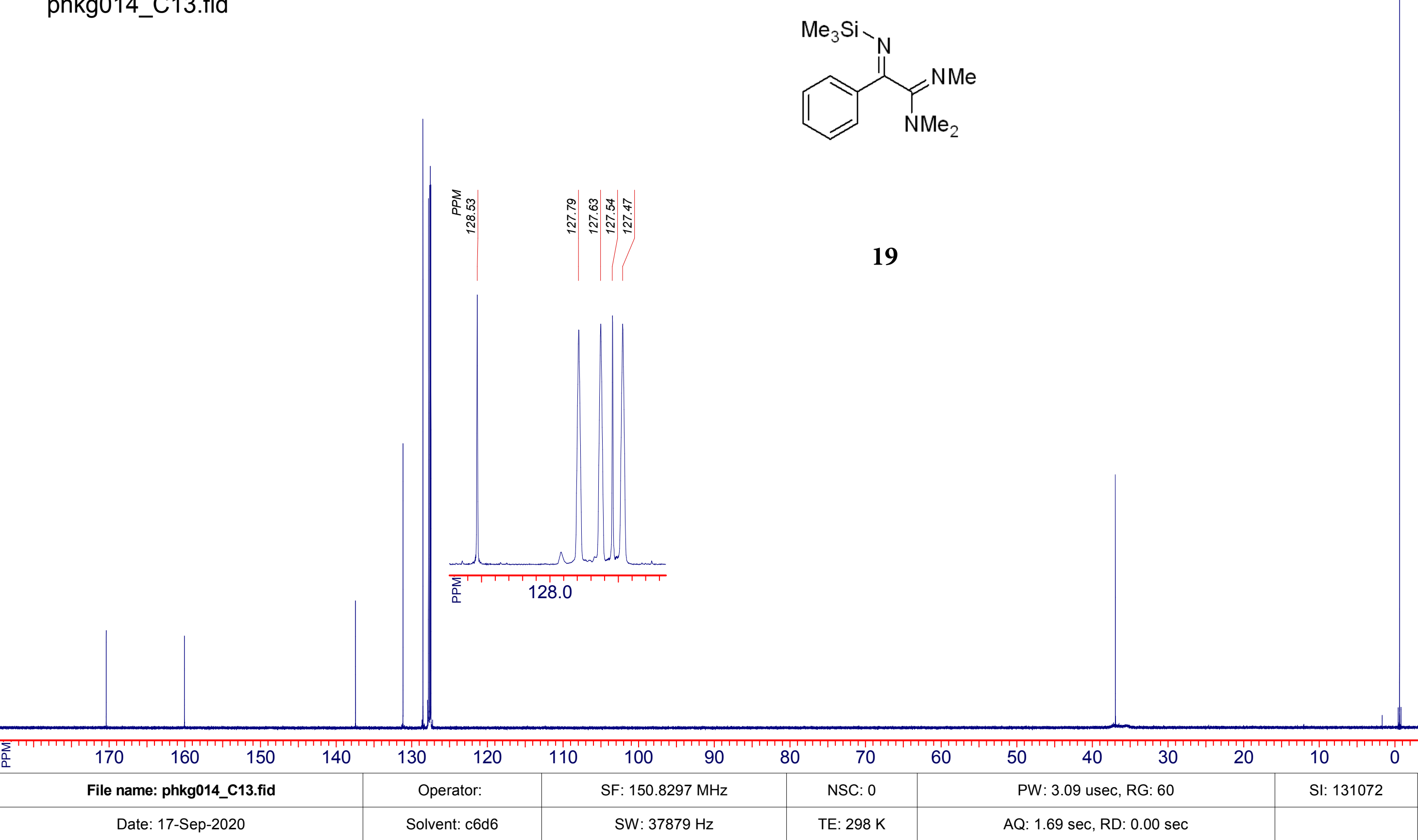




\section{phkg014_C13APT.fid}
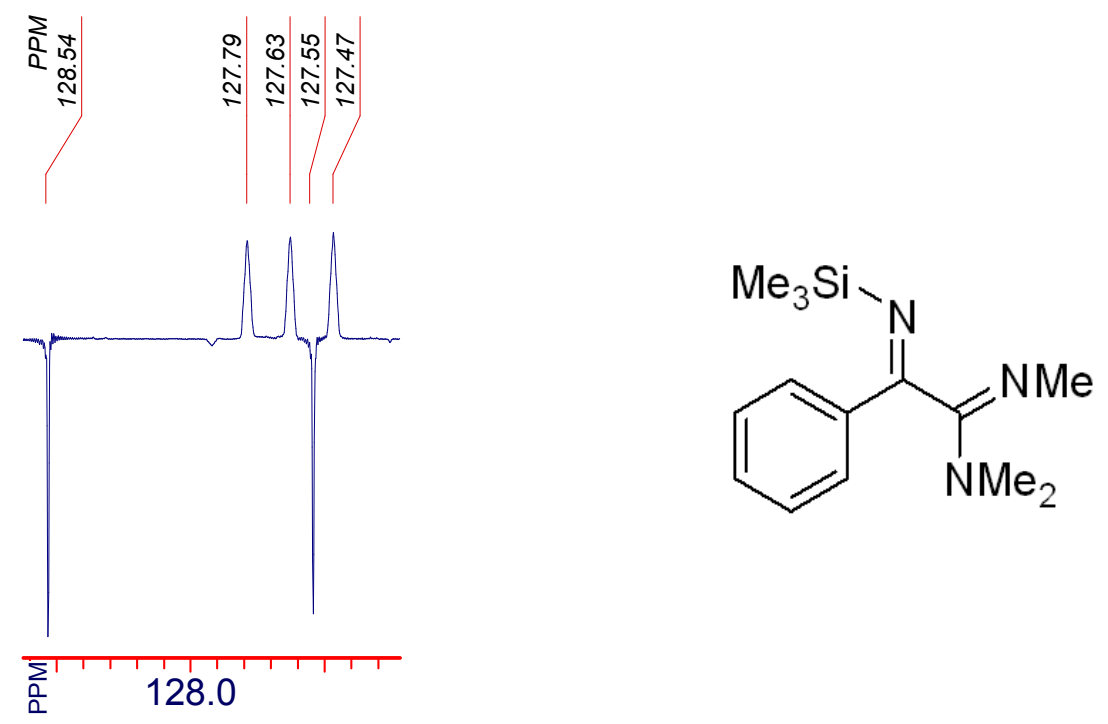

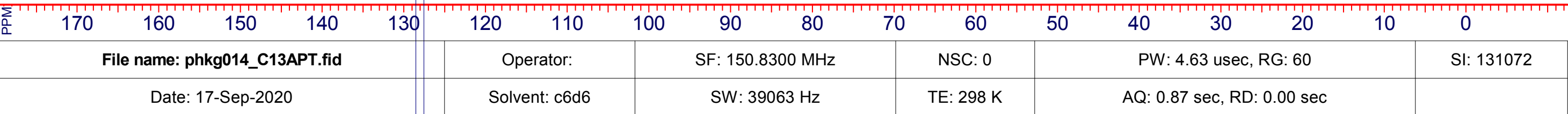




\section{phkg003_C13.fid}

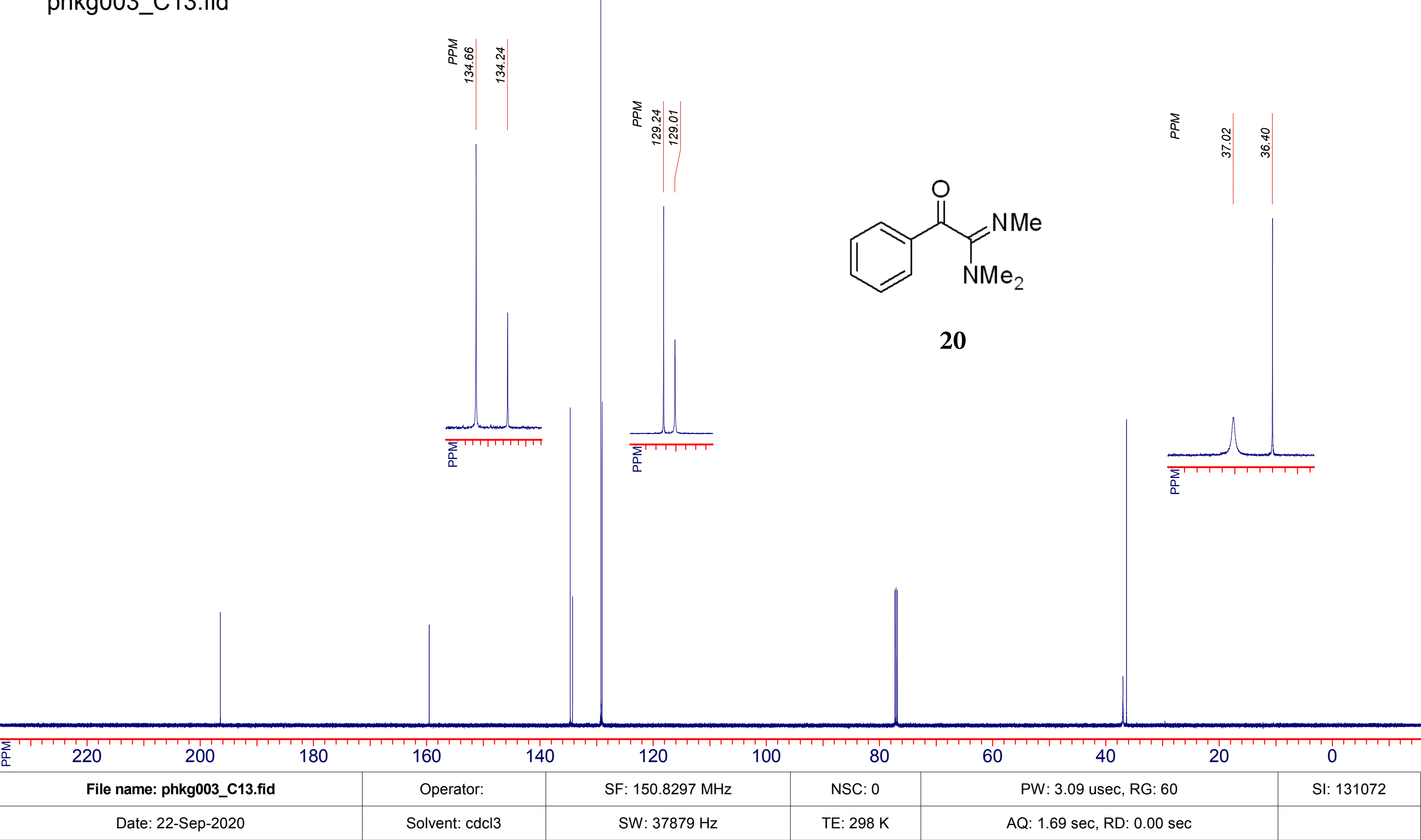




\section{phkg003_C13APT.fid}

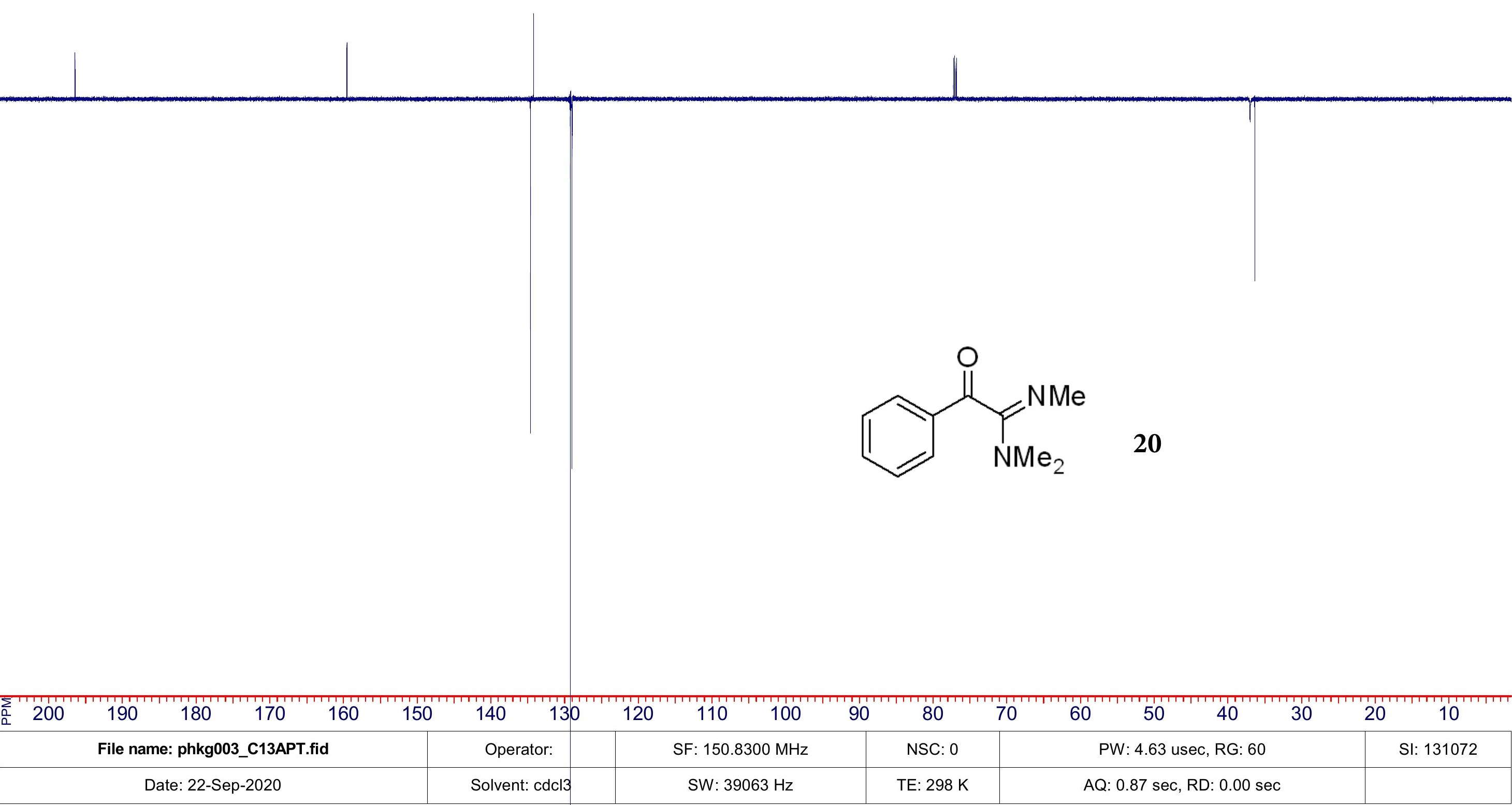


phkg003.fid
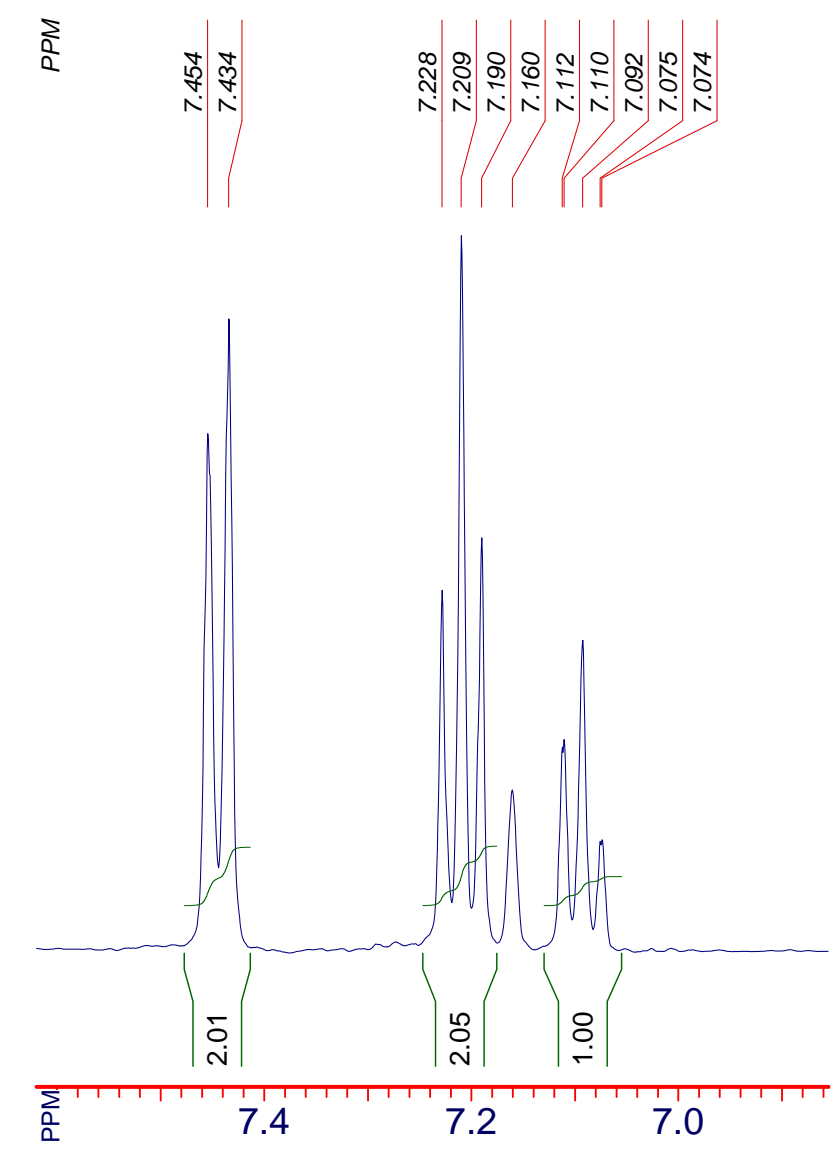

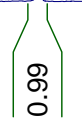

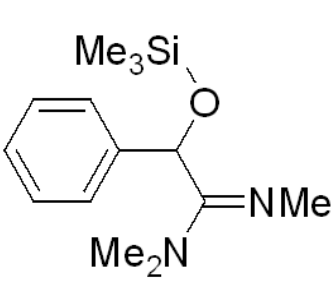

21

$\sum_{\substack{0 \\ 0}}^{\sum_{1}+1}$

$8.0 \quad 7.5$

6.0

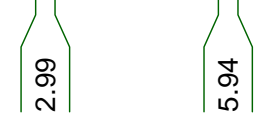

File name: phkg003.fid

Operator:

5.5

$5.0 \quad 4.5$

$4.5 \quad 4.0$

3.5

$3.0 \quad 2.5$

$2.5 \quad 2.0$

$2.0 \quad 1.5$

$1.5 \quad 1.0$

PW: 11.60 usec, RG: 26

AQ: $2.11 \mathrm{sec}, \mathrm{RD}: 0.00 \mathrm{sec}$ 


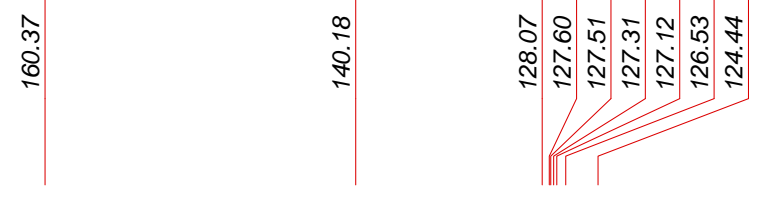

\section{phkg003_C13}

$\frac{5}{5}$

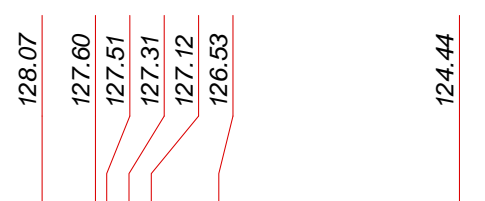<smiles>CNC(=[18O])C(O[SiH3])c1ccccc1</smiles>

21

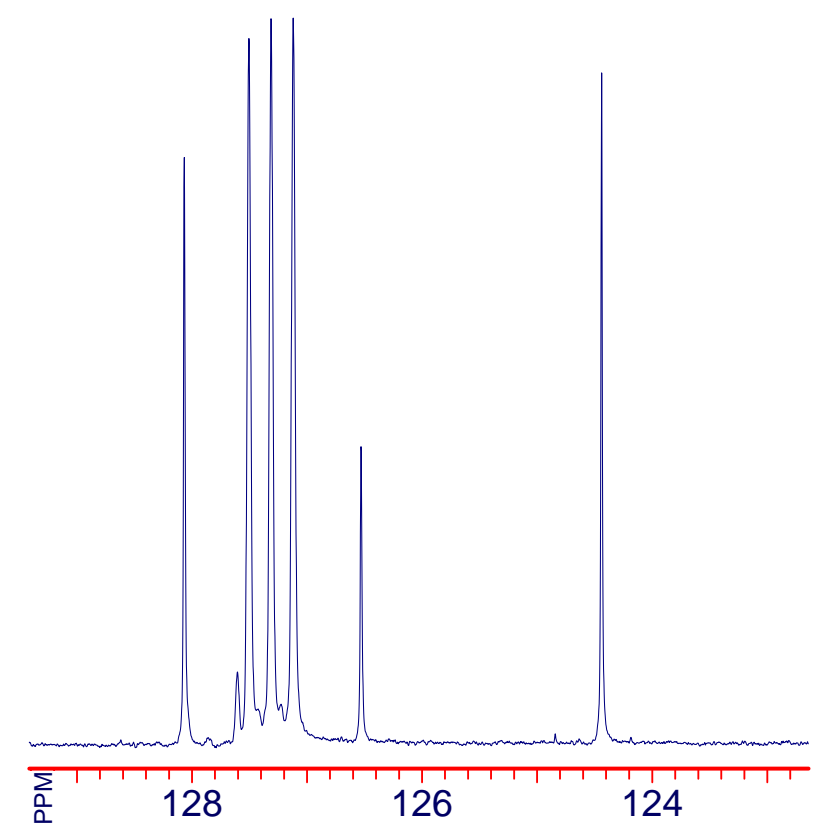

का

1701601501111111111111111111111

File name: phkg003_C13

$140130 \quad 120$

$110 \quad 100$

90

80

60

50

$40 \quad 30$

30

20

10

0

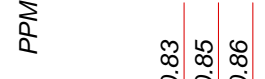

\begin{tabular}{l|l|l|}
$\Re$ & 1 & 0 \\
$\infty$ & $\infty$ & 0 \\
0 & 0 & 0
\end{tabular}

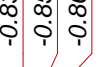

Operator: root

SF: $125.6681 \mathrm{MHz}$

PW: 0.00 usec, RG: 51200

SI: 131072 



\section{PHKG017_C13APT}<smiles>C[SiH2]OP1CCCC1</smiles>

22

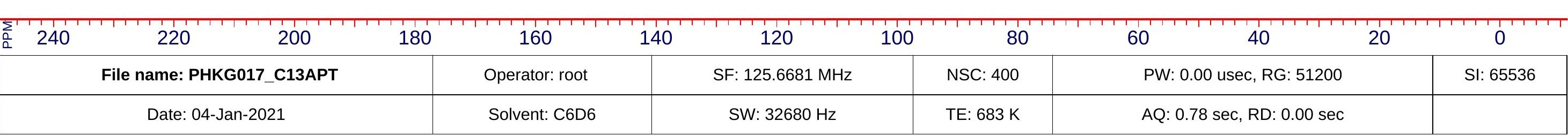




\section{PHKG017_P31-H.fid}

$\frac{5}{8}$<smiles>C[SiH2]OP1CCCC1</smiles>

22

$220 \quad 200 \quad 180 \quad 160 \quad 140 \quad 120$

$120 \quad 100$

$80 \quad 60 \quad 40 \quad 20$

$20 \quad 0 \quad-20 \quad-40$

$-60 \quad-80$

$-100$

Operator:

SF: $202.3355 \mathrm{MHz}$

NSC: 0

SW: $83333 \mathrm{~Hz}$

TE: $298 \mathrm{~K}$

PW: 4.00 usec, RG: 30

AQ: $1.64 \mathrm{sec}, \mathrm{RD}: 0.00 \mathrm{sec}$ 


\section{phkg008.fid}
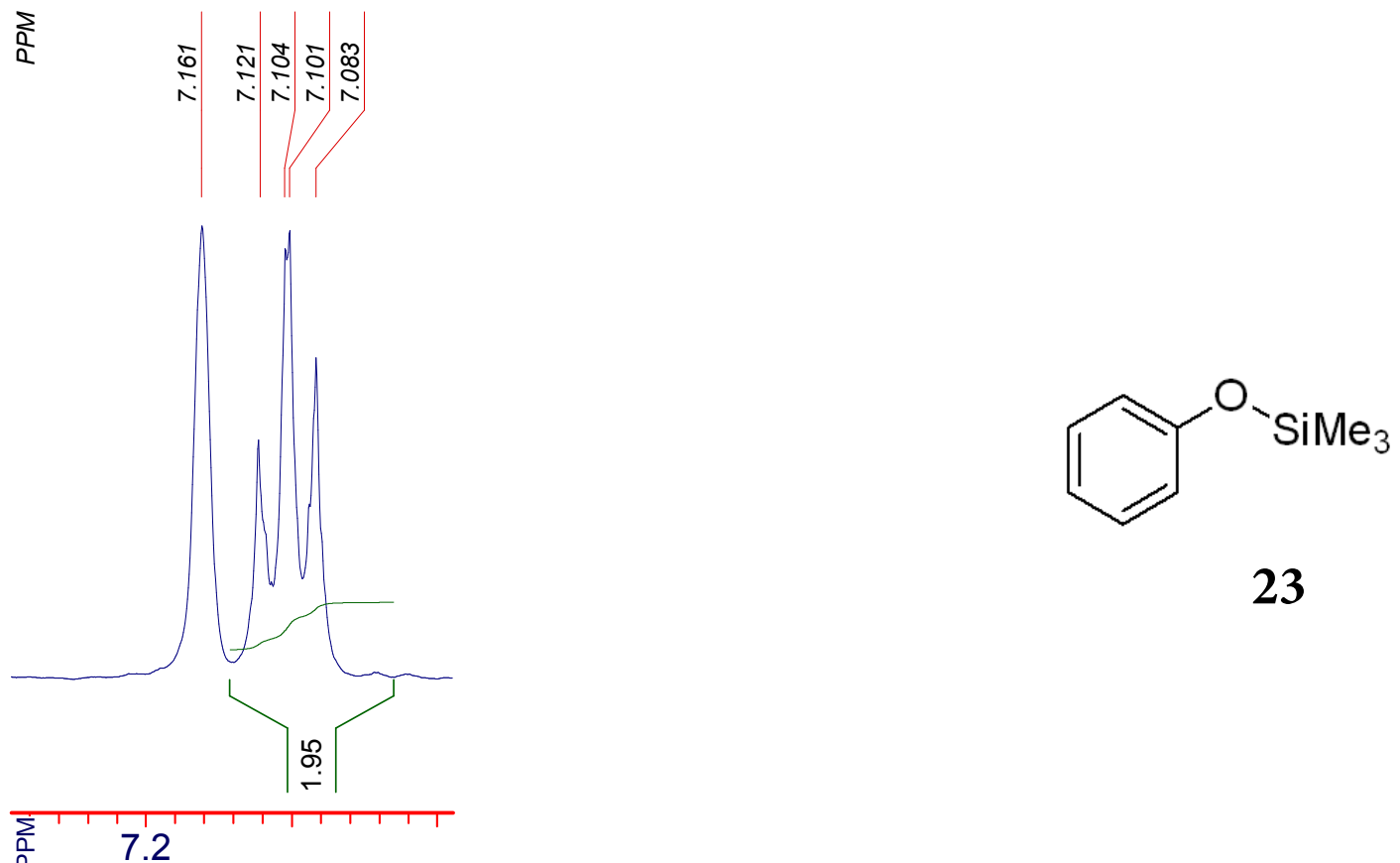

23
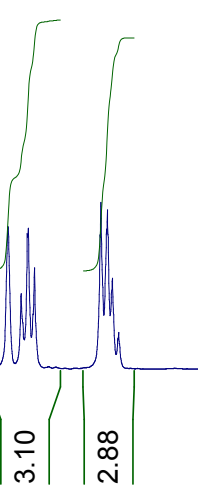

7.5

7.0

6.5

5.5

5.0

4.5

4.0

:

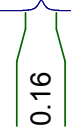

File name: phkg008.fid

SF: $399.9736 \mathrm{MHz}$

3.5

3.0

2.5

2.0

PW: 11.60 usec, RG: 26

$A Q: 2.11 \mathrm{sec}, \mathrm{RD}: 0.00 \mathrm{sec}$ 


\section{phkg008_C13}

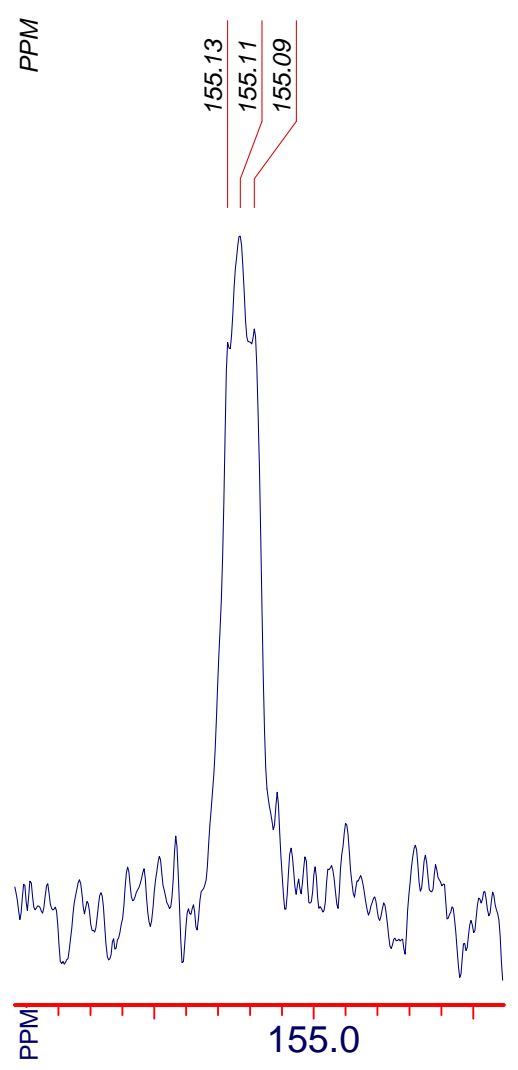

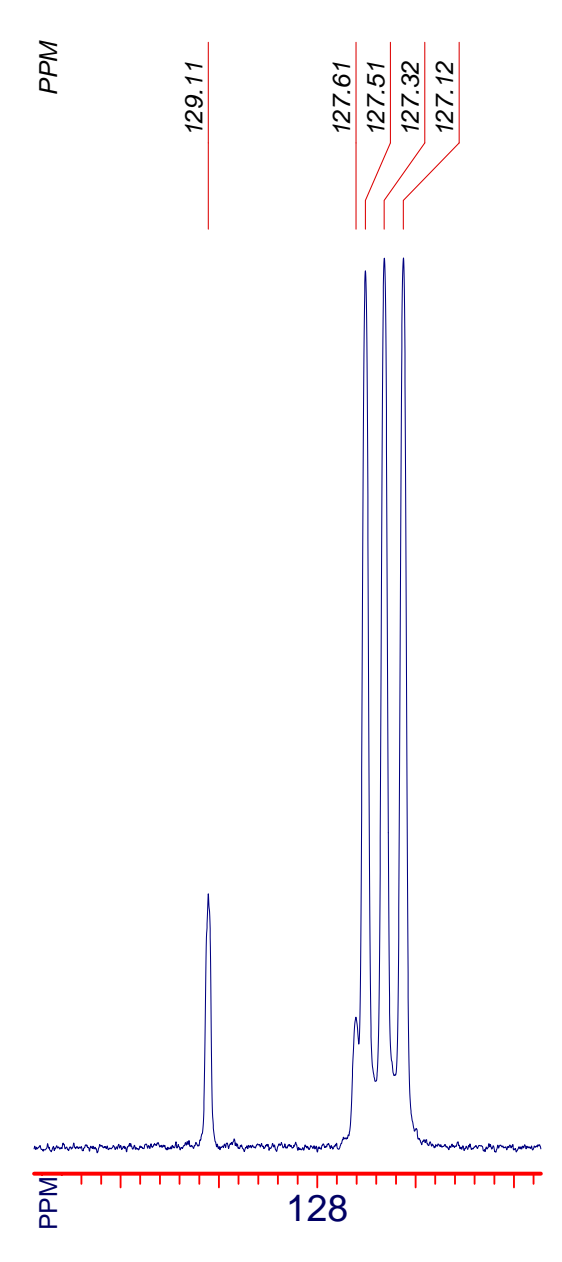

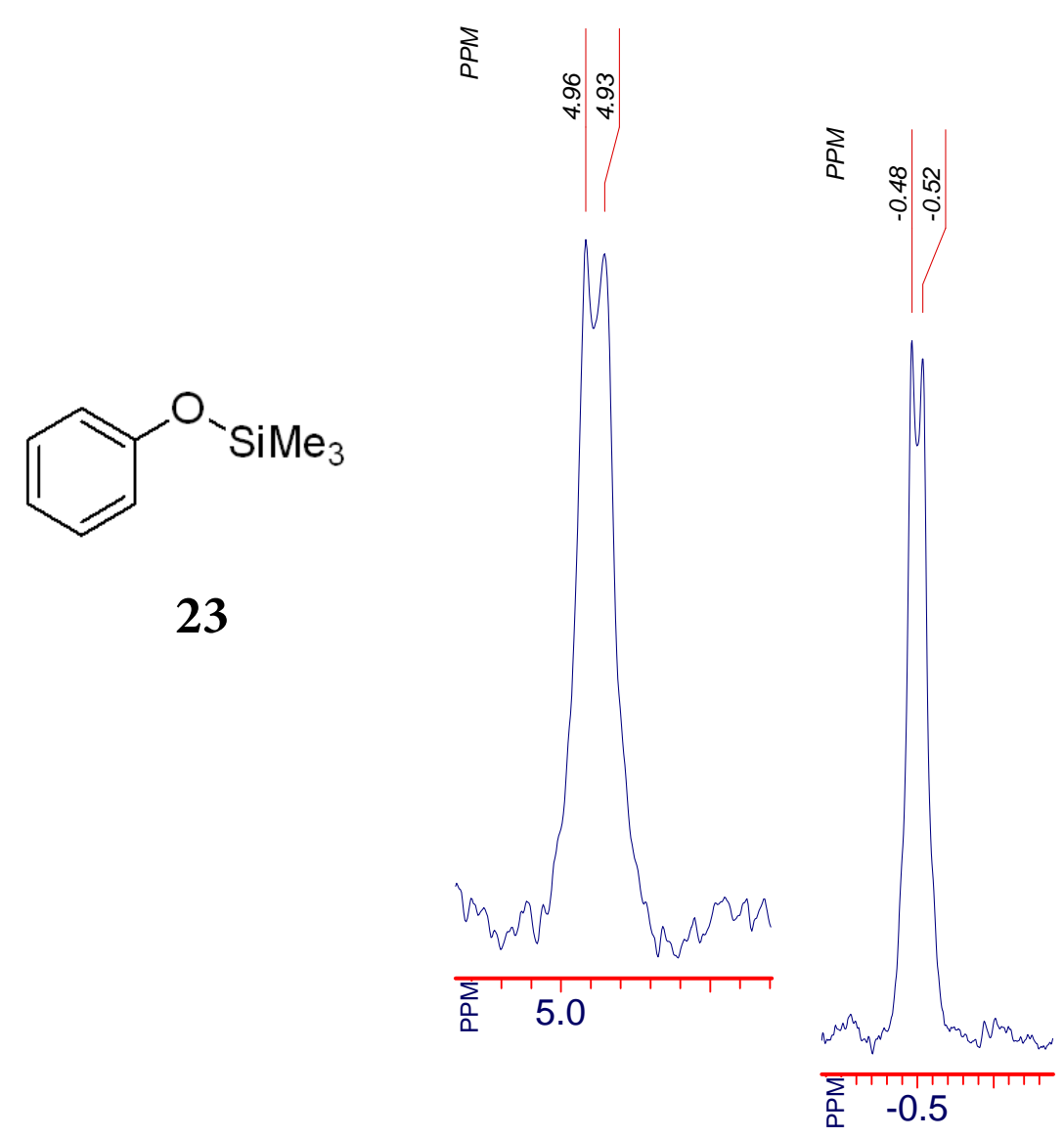

23

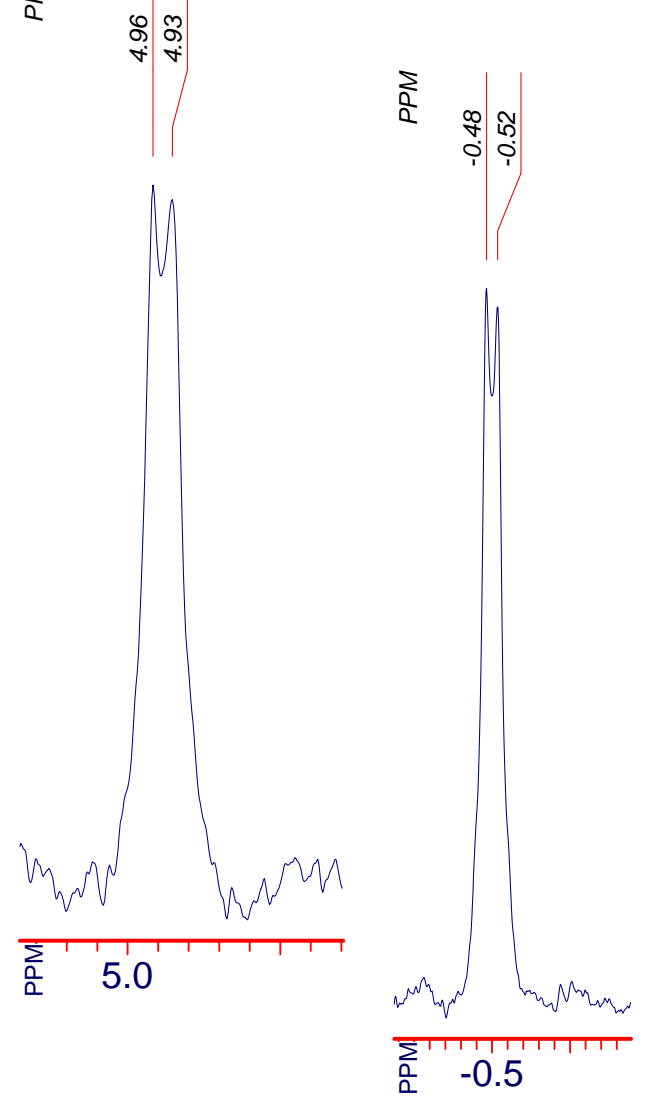

$\sum_{n=11}$
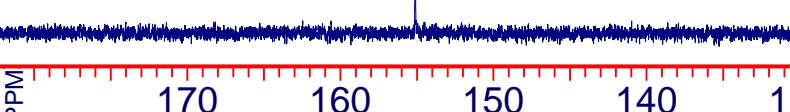

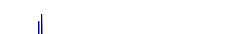

File name: phkg008_C13

120

110

$100 \quad 90 \quad 80$

70

$60 \quad 50$

$50 \quad 40$

30

$20 \quad 10$

NSC: 150 PW: 0.00 usec, RG: 51200

TE: $683 \mathrm{~K}$

AQ: $1.57 \mathrm{sec}, \mathrm{RD}: 0.00 \mathrm{sec}$

\begin{tabular}{ll} 
& \\
\hline
\end{tabular}




\section{phkg007.fid}
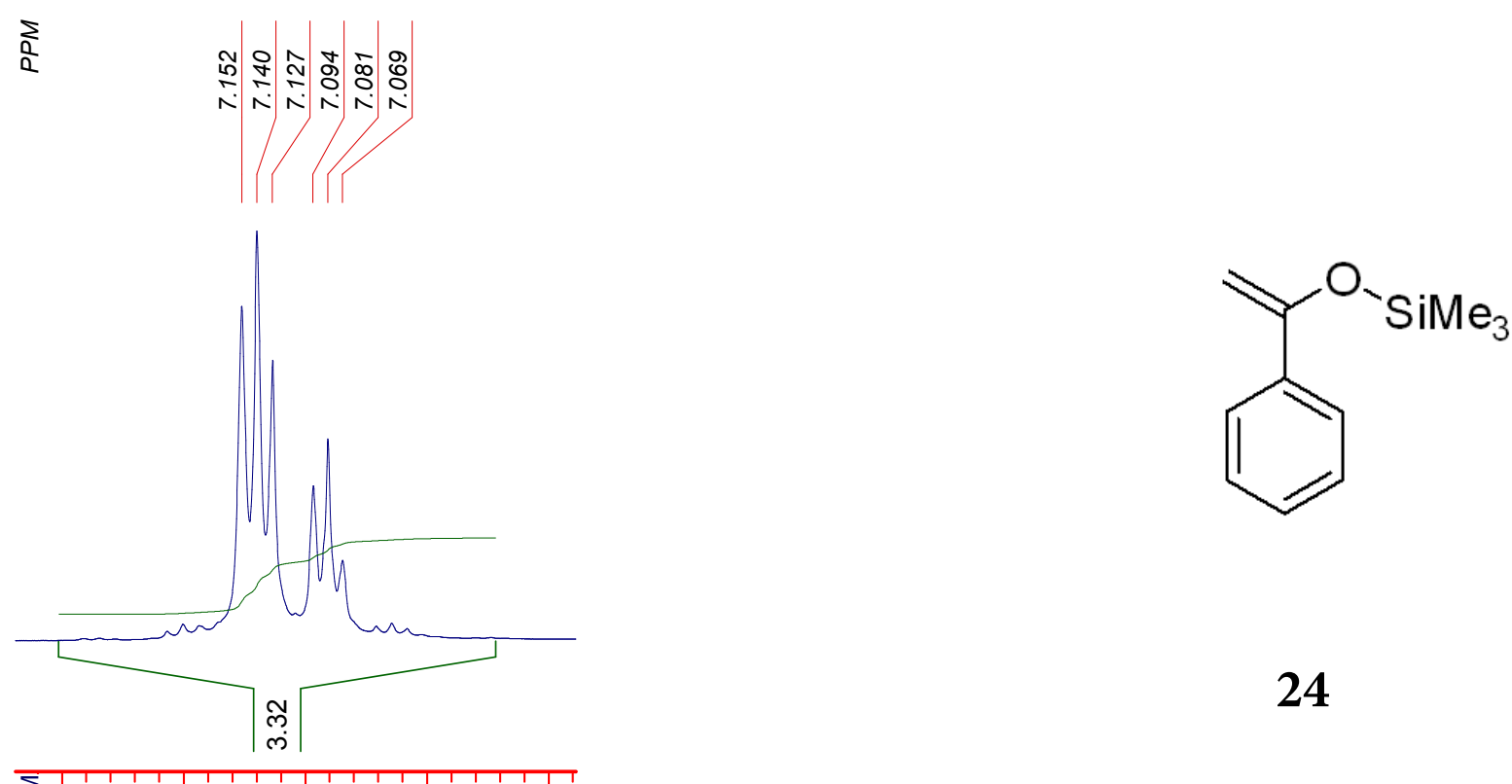

24
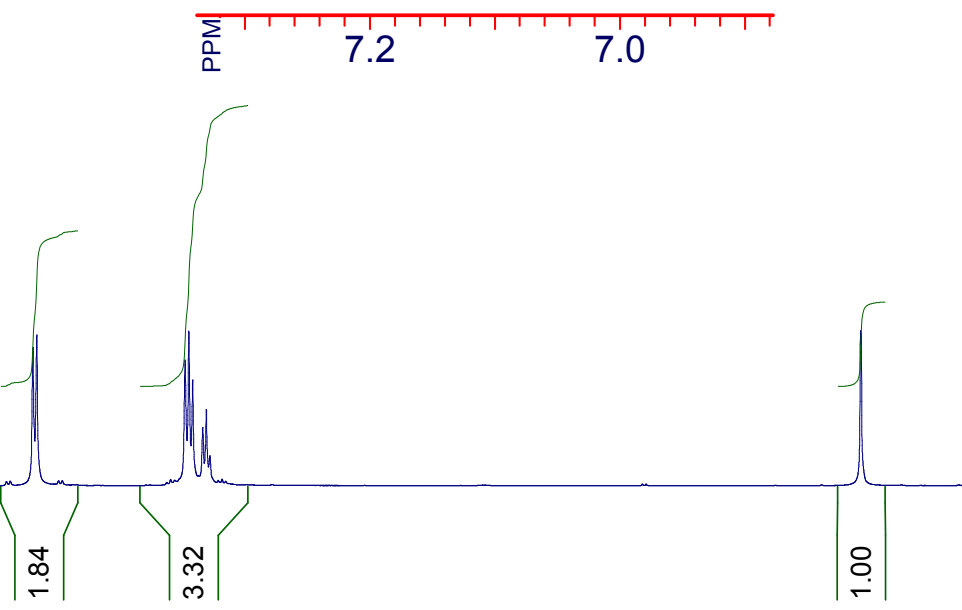

$8.0 \quad 7.5$

$5.5 \quad 7.0$

6.5

6.0

5.5

5.0

$\left|\begin{array}{l}\infty \\ \infty \\ 0 \\ 0\end{array}\right|$

File name: phkg007.fid

Operator:

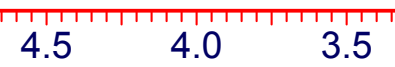

$\left|\begin{array}{l}\infty \\ \infty \\ 0 \\ 0\end{array}\right|$

$\infty$
$\dot{0}$$\left|\begin{array}{l}\text { g } \\ \dot{0}\end{array}\right|$ 


\section{phkg007_C13.fid}
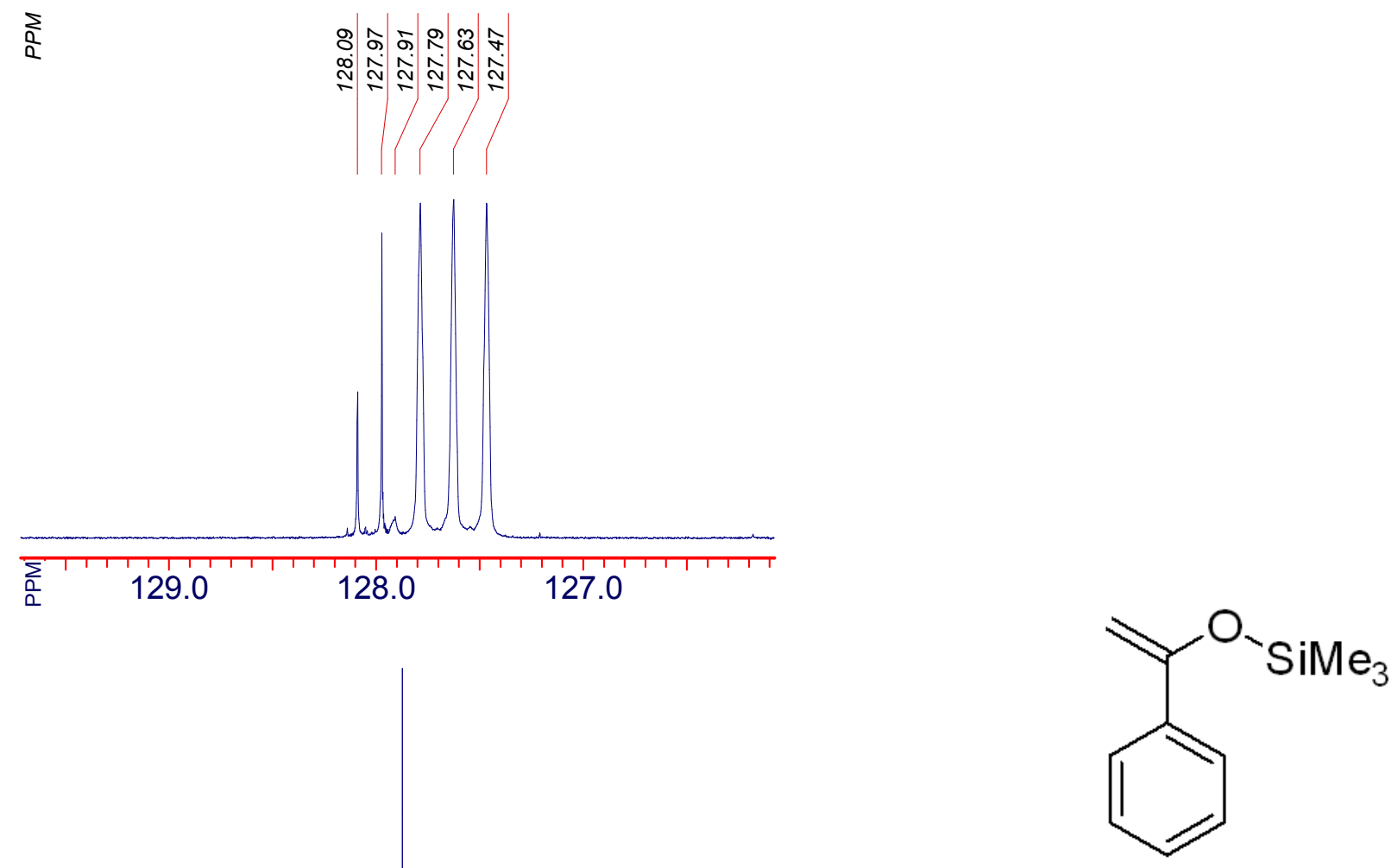

24

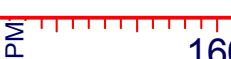

$160 \quad 150$

140

130

120

110

100

90

80

70

File name: phkg007 C13.fid

Operator:

SF: $150.8297 \mathrm{MHz}$

Solvent: c6d6

PW: 3.09 usec, RG: 60

20

10 
phkg007_C13APT.fid

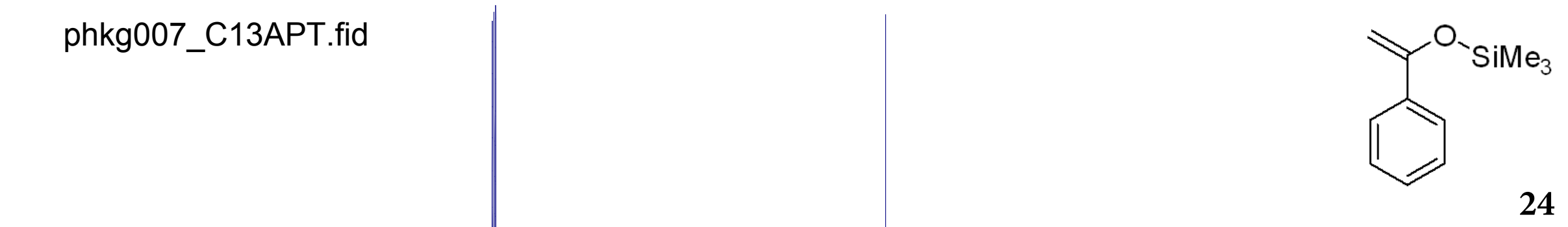




\section{PHKG023_C13.fid}

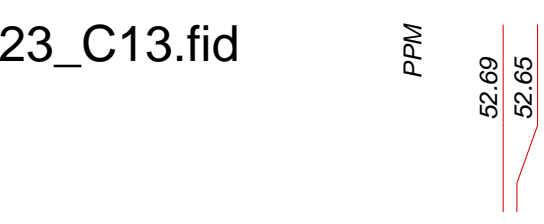

$\frac{8}{2}$

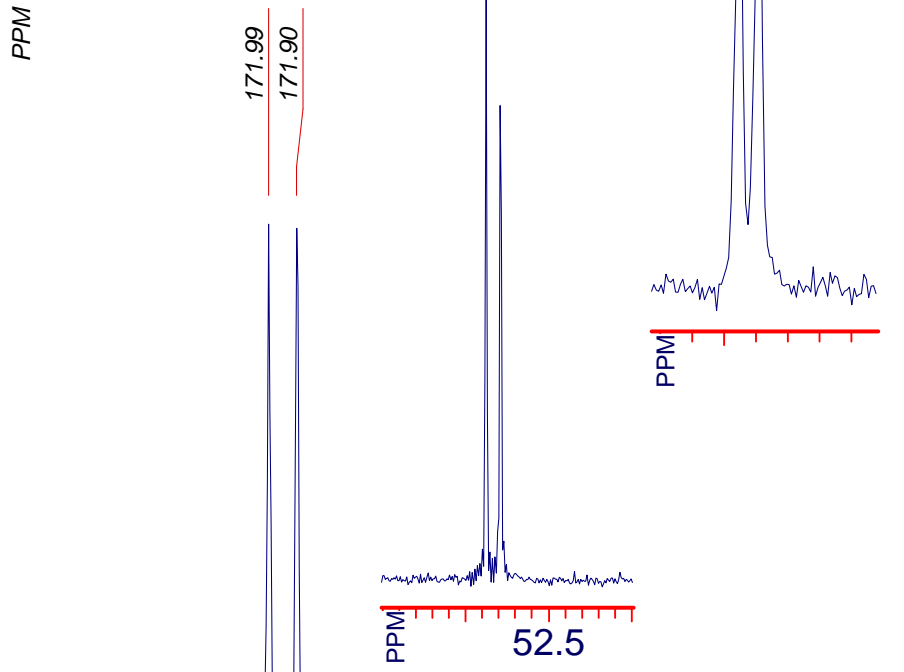

$\sum_{\text {임 }} 172.5$

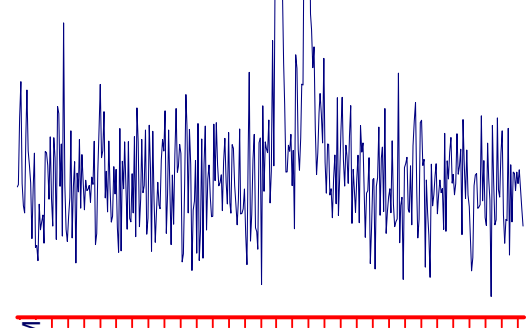

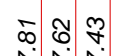

ㅇำ $\approx$

S146

$\frac{2}{2}$

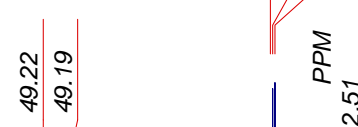

นึ่

$\stackrel{\sim}{\sim}$

l

$\mid \begin{gathered}\mathrm{Me}_{3} \mathrm{SiO}_{\mathrm{O}} \mathrm{OSiMe}_{3} \\ \mathrm{MeO}-\mathrm{P}=\mathrm{C}=\mathrm{C}^{\prime} \\ \mathrm{MeO}\end{gathered}$

26

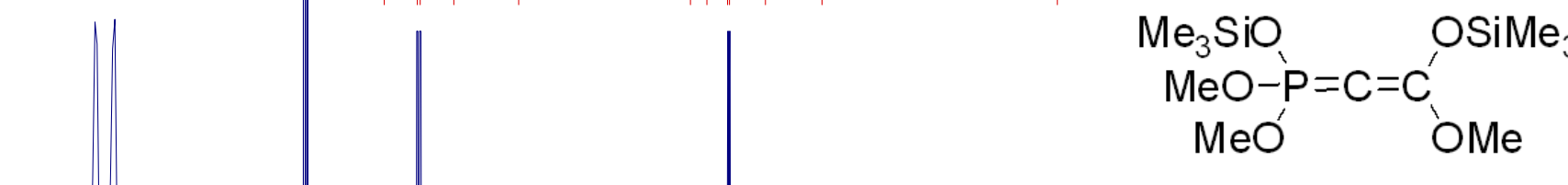

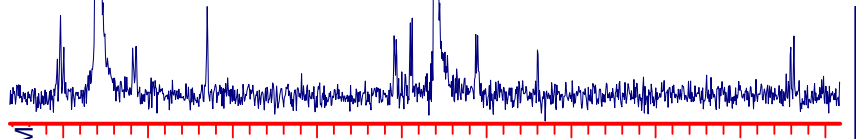
$\begin{array}{lllll}\text { हो } 2.0 & 1.0 & 0.0 & -1.0\end{array}$

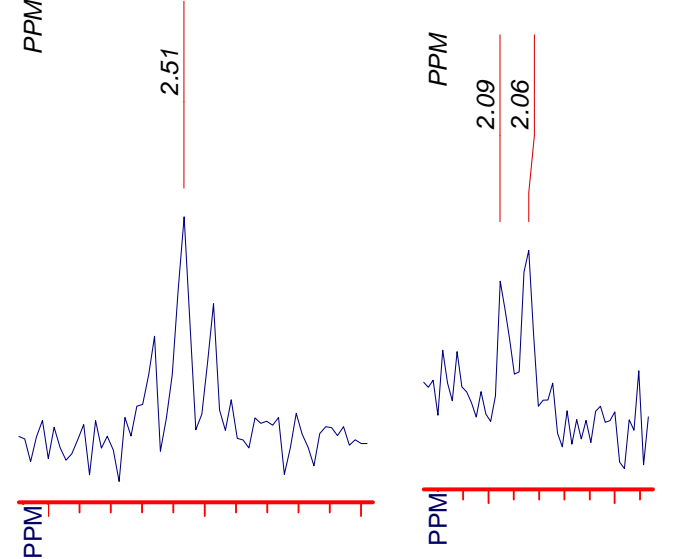

袙

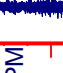

$220 \quad 200$
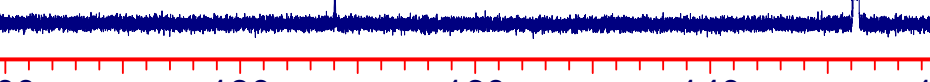

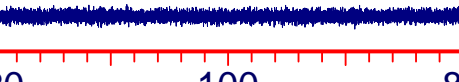

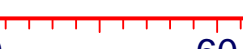

$60+1+1+1+1+10$

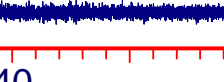

File name: PHKG023_C13.fid

Operator:

SF: $125.6921 \mathrm{MHz}$

NSC: 0

PW: 3.27 usec, RG: 60

20

0 
PHKG023_P31-H.fid

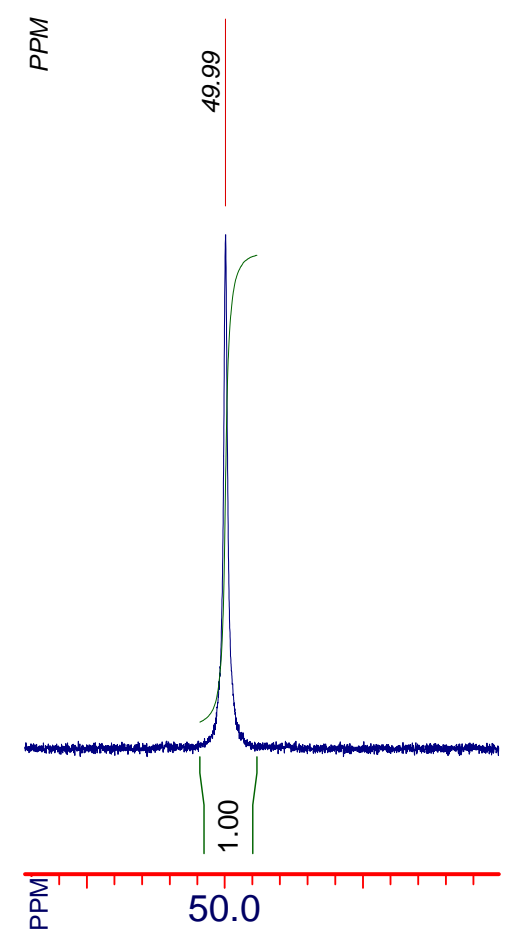

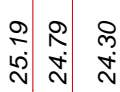

(

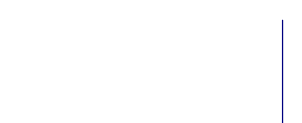

$\mathrm{Me}_{3} \mathrm{SiO}_{\mathrm{MeO}}^{\mathrm{P}}=\mathrm{C}=\mathrm{C}_{\mathrm{C}}^{\mathrm{C}^{\prime}}$
$\mathrm{OSiMe}_{3}$

26
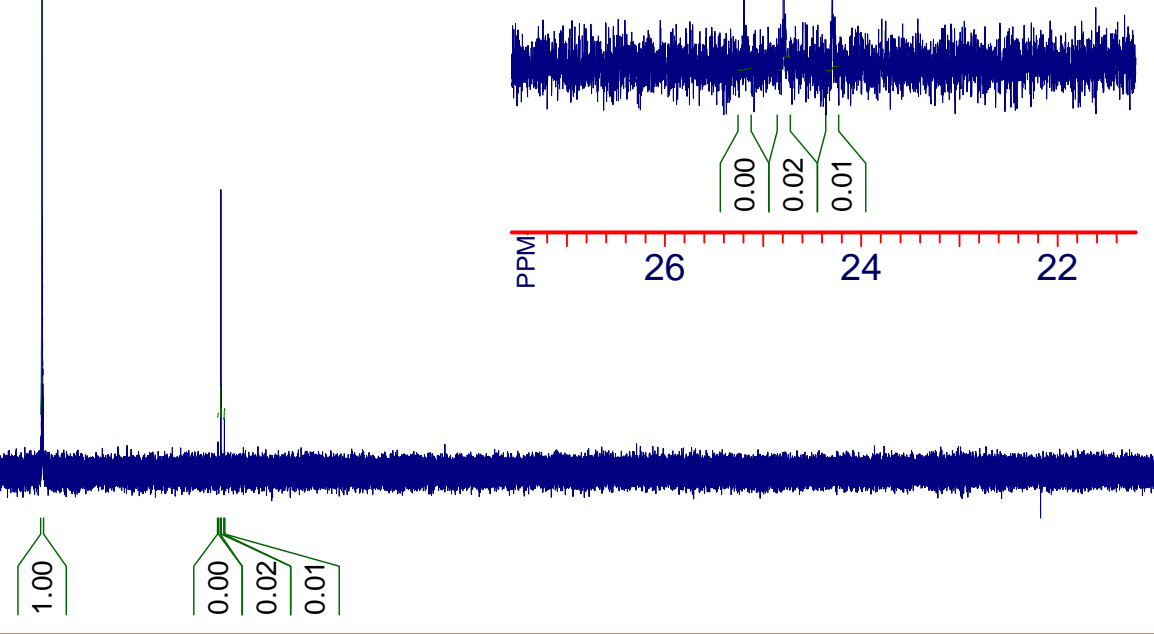

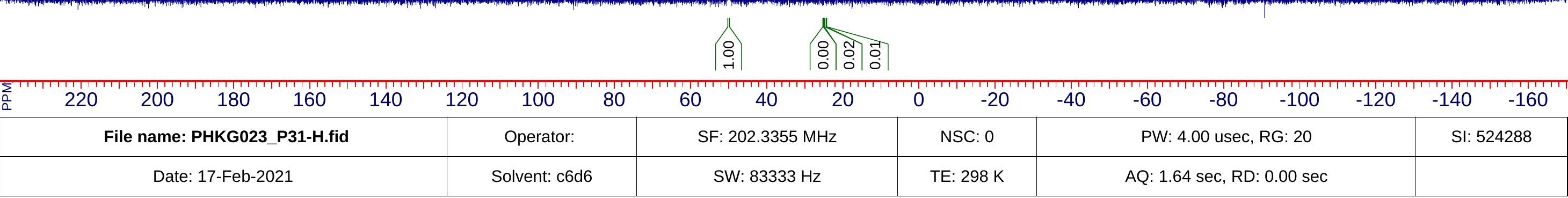

